\title{
Cosmic Ray Muon Charge Ratio in the MINOS
}

\section{Far Detector}

\author{
by \\ Erik. B. Beall \\ B.A., Saint Olaf College, 2000
}

\author{
A thesis submitted to the \\ Faculty of the Graduate School of the \\ University of Minnesota in partial fulfillment \\ of the requirements for the degree of \\ Doctor of Philosophy \\ Department of Physics and Astronomy
}

December, 2005 
This thesis entitled:

Cosmic Ray Muon Charge Ratio in the MINOS Far Detector written by Erik. B. Beall

has been approved for the Department of Physics and Astronomy

Marvin Marshak

Maury Goodman

Date

The final copy of this thesis has been examined by the signatories, and we find that both the content and the form meet acceptable presentation standards of scholarly work in the above mentioned discipline. 


\section{Dedication}

To Courtney and all the rest of my family. Courtney, it has been one strange and wonderful road, and since the day we met, its become more wonderful, my life has become so much richer, and my love for life has grown with my love for you. 


\section{Acknowledgements}

I owe so much to so many without whom this work would never have been completed or even begun. Mom and Dad, you nurtured my interest in the world around me and taught me that fascinating things were happening all around us, we

just had to open our eyes. Peter, you've been a great brother and friend. Keith, keep making that music. Thanks both of you for listening to my crazy explanations and for being there and being some strange and fun people to hang out with. Many thanks to the rest of my family, you've all supported me over the years and I really appreciate it. A special thanks to my new family of in-laws who have also encouraged me, kept me entertained, and fed me over the past year and a half, thanks Mom and Dad James.

Maury, thanks for giving me the confidence to think for myself in a new field of endeavour. And thanks for putting up with my crazy, sometimes on-the-spot explanations! Joe, thanks for being a great room-mate and friend. I'll remember the fun at Cecil street, as will many others, for many years. Marvin, you were always such a great supporter, tell you what; I'll pay your bar tab for a night... To all the guys on the experiment: Tom, Ben, Alex, Jason, Brian, Rustem, Dan, Caius, Phil, Simona, Rodolphe, Tass, Dipu, Jeremy, Andrew, and all the rest; all the great times and conversations up at the mine, at fermilab and over in Switzerland, I keep fond memories of all of you. All the guys in my office: I hope you all make it out of there soon! Even if its just down the hall. Thanks to all who came by and filled 
our lives with foosball daily, Steve, Tao, Emily, the guy on the couch, anyone else who happened by. Thanks for finally getting the foosball table out of our office so we could do some work.

Thanks to all the professors and mentors I've had who taught me to think for myself, Lisa Irkhe, Glen Skov (yes, I really did appreciate your class), Connie Nelson, Mat Murphy, Dave Nitz, Jim Cederberg, Greg Olsen, Dave and Bob Hauck, the crazy guy at St Olaf who taught aesthetics, Alexander Grosberg, Paul Crowell, and all the first year physics teachers around. You've all taught me so much, its really unfortunate that I've waited to become ready to learn until now, a mistake I'll never make again! 
Beall, Erik. B. (Ph.D., Physics)

Cosmic Ray Muon Charge Ratio in the MINOS Far Detector

Thesis directed by Prof. Marvin Marshak and Dr. Maury Goodman

The MINOS Far Detector is a 5.4 kiloton $(5.2 \mathrm{kt}$ steel plus $0.2 \mathrm{kt}$ scintillator plus aluminum skin) magnetized tracking calorimeter located 710 meters underground in the Soudan mine in Northern Minnesota. MINOS is the first large, deep underground detector with a magnetic field and thus capable of making measurements of the momentum and charge of cosmic ray muons.

Despite encountering unexpected anomalies in distributions of the charge ratio $\left(N_{\mu^{+}} / N_{\mu^{-}}\right)$of cosmic muons, a method of cancelling systematic errors is proposed and demonstrated. The result is $R_{e f f}=1.346 \pm 0.002$ (stat) \pm 0.016 (syst) for the averaged charge ratio, and a result for a rising fit to slant depth of $R(X)=$ $1.300 \pm 0.008$ (stat) \pm 0.016 (syst) $+(1.8 \pm 0.3) \times 10^{-5} \times X$, valid over the range of slant depths from $2000<\mathrm{X}<6000$ MWE. This slant depth range corresponds to minimum surface muon energies between $750 \mathrm{GeV}$ and $5 \mathrm{TeV}$. 


\section{Contents}

\section{Chapter}

1 Introduction 1

2 Cosmic Ray Muons $\quad 6$

2.1 Cosmic Ray Muon Origin . . . . . . . . . . . . . . . 6

2.1.1 Cosmic Ray Primaries . . . . . . . . . . . . . . . . 6

2.1.2 Primary Interaction with Atmosphere . . . . . . . . . 7

2.1 .3 Hadron Production . . . . . . . . . . . . . . . . . . . . 9

2.2 Charge Ratio . . . . . . . . . . . . . . . . 10

2.3 Cosmic Ray Muons . . . . . . . . . . . . . . . . . . . . . 13

2.3.1 Energy Spectrum . . . . . . . . . . . . . . 13

$2.3 .2 \quad$ Energy Loss . . . . . . . . . . . . . . . . . . . . . . 14

2.3.3 Charge Dependence of Energy Loss . . . . . . . . . . . . . . . 19

2.3 .4 Scattering . . . . . . . . . . . . . . . . . 19

2.3.5 Propagation Through Rock . . . . . . . . . . . . . . 19

2.4 Expected at MINOS . . . . . . . . . . . . . . . 20

2.4 .1 Intensity Expected . . . . . . . . . . . . 20

2.4.2 Charge Ratio Expected ............... 22 
3 Previous Measurements of the Charge Ratio 23

3.1 Surface Measurements . . . . . . . . . . . . . . . . . . 23

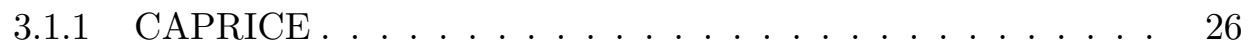

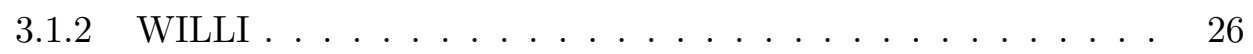

3.2 Underground Experiments . . . . . . . . . . . . . . . . . . 28

$3.2 .1 \quad$ L3 Experiment . . . . . . . . . . . . . . . . 30

3.2 .2 Cosmo-ALEPH Experiment . . . . . . . . . . . 31

4 The MINOS Far Detector 32

4.1 Construction of the Detector . . . . . . . . . . . . . 34

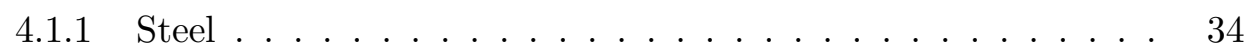

4.1 .2 Scintillator . . . . . . . . . . . . . . 38

4.1.3 Completion of Supermodules . . . . . . . . . . . . 45

4.2 Detector Readout and DAQ . . . . . . . . . . . . . . 47

4.2 .1 DAQ Triggers ..................... 53

4.3 Reconstruction ......................... 54

4.3 .1 Calibration ...................... 55

4.3 .2 Track Finding. . . . . . . . . . . . . . . . 55

4.3 .3 Track Fitting . . . . . . . . . . . . . . . 57

4.4 Coordinate Systems _ . . . . . . . . . . . . . . . 60

4.5 Typical Tracks . . . . . . . . . . . . . . 66

5 Cosmic Ray Muons in MINOS $\quad 71$

5.1 Data and Monte Carlo Cuts . . . . . . . . . . . . . . 71

5.2 Detector Acceptance and Efficiency . . . . . . . . . . . . . 72

5.2.1 Monte Carlo Generation . . . . . . . . . . . . . . . 74

5.3 The Data . . . . . . . . . . . . . . . . 75 
5.3.1 Detector Axis-Centered Coordinates . . . . . . . . . . . 84

5.3 .2 Data Purity Cut . . . . . . . . . . . . . . . 84

5.4 MINOS Cavern Rock Map . . . . . . . . . . . . . . . . 86

5.5 Examination of Muon Behavior in the Far Detector . . . . . . . . . . 87

5.5.1 High Energy, or Straight, Muons . . . . . . . . . . . . 87

5.5.2 Low Energy Muons, or Curvature in the Far Detector . . . . 90

6 Charge Determination in MINOS 96

6.1 Summary of the Problem . . . . . . . . . . . . . . . . 96

6.2 Magnetic Field . . . . . . . . . . . . . . . . . . . . . . 108

6.2.1 An Argument Against the Field Being the Problem . . . . . . 108

6.2 .2 Toy Monte Carlo . . . . . . . . . . . . . . . . . . . . . . 109

6.3 Alignment . . . . . . . . . . . . . . . . . . . . 110

6.3.1 Longitudinal, or Z-axis Alignment . . . . . . . . . . 110

7 Measurement of the Charge Ratio 116

7.1 Charge Biases in the Data . . . . . . . . . . . . . . . 116

7.2 Summing the Complementary Data Sets . . . . . . . . . . . . . . . . 119

7.2.1 Monte Carlo and Reweighting . . . . . . . . . . . . . . 121

7.3 Slant Depth . . . . . . . . . . . . . . . . . . . . 124

7.4 Surface Energy . . . . . . . . . . . . . . . . . . . . . . . . 124

8 Systematic Error 128

8.1 Time Dependence of Charge Ratio Measurement . . . . . . . . . . . 129

8.2 Analysis Cuts Dependence of Charge Ratio Measurement . . . . . . 131

8.3 Conclusion . . . . . . . . . . . . . . . . . . . 131 


\section{Tables}

\section{Table}

1.1 Symbols Used . . . . . . . . . . . . . . . . . .

3.1 A Compilation of Charge Ratio Experiments and their Results and Specifics. . . . . . . . . . . . . . . . 31

5.1 Effect of Incremental Cuts on Forward Field Data Sample. . . . . . . 72

5.2 Effect of Incremental Cuts on Reverse Field Data Sample. . . . . . . 73

5.3 Effect of Incremental Cuts on Monte Carlo Sample. . . . . . . . . . . 73

5.4 Data Runs and events analyzed, dates. Note that Run Number Ranges are not consecutive and may have large gaps. . . . . . . . . . 80

5.5 The expected curvature based on simplified detector and a track length of 8 meters: parallel to z-axis is d cosz $=1.0$, perpendicular is 0.0 . For comparison, a strip's dimensions are 0.01 by 0.041 meters. . . . . . . . . . . . . . . . . .

7.1 The $\chi^{2}$ of a fit to the Charge Ratio versus Detector Azimuth for Forward and Reverse field data, and also after the summing procedure.118 


\section{Figures}

Figure

1.1 A cartoon of a cosmic ray air shower, with remnant particles extending into the Earth over the Soudan Underground Laboratory. . . . .

1.2 Compilation of recent experimental results, with world fit overlain and CORT predictions in red line (from Naumov). . . . . . . . .

2.1 Caption and Figure taken from [1], data points from [2]. . . . . . 8

2.2 Kaon production diagrams for $p p \rightarrow p, n Y K$ reaction channels. . . . 11

2.3 Kaon production diagrams for $p n \rightarrow p, n Y K$ reaction channels. . . . 11

2.4 Muon rates, taken from $[1] \ldots \ldots \ldots \ldots \ldots$

2.5 Muon survival curves as function of original energy for various slant depths of rock $[3] . \ldots \ldots \ldots \ldots \ldots \ldots$

2.6 Histogram of surface energy for measured (low energy) muons based on overburden and Equation 2.11. . . . . . . . . . . 18

2.7 Muon intensity versus slant depth, taken from [4]. Slant depths corresponding roughly to particular zenith angles from the Soudan hall are marked and are valid for MINOS site. . . . . . . . . . . .

3.1 Compilation of recent experimental results, with world fit overlain and CORT predictions in red line (from Naumov). . . . . . . 
3.2 Compilation of recent charge ratio measurements, from Hebbeker and Timmermans. . . . . . . . . . . . . . . . . . 25

3.3 Charge Ratio versus momentum for CAPRICE. . . . . . . . . . . . 27

3.4 Charge Ratio versus momentum for the two experiments WILLI and CAPRICE, esp CAPRICE97 data, from [5] . . . . . . . . . . . . . 29

3.5 Charge Ratio versus momentum, data taken from tables in [6]. . . . 30

4.1 The MINOS Far Detector in graphical view. . . . . . . . . . . . 33

4.2 The MINOS Far Detector in the cavern hall. . . . . . . . . . . . . 35

4.3 Construction of the MINOS Far Detector, two adjacent planes. The Southern plane is in the steel lamination phase, the Northern station has finished its steel lamination and welding phase and has begun to have its first scintillator module welded in place. . . . . . . . . 35

4.4 Steel Laminations of a steel plane. . . . . . . . . . . . . . . . . 37

4.5 Magnetic Field in a steel plane. . . . . . . . . . . . . . . . . . 39

4.6 Scintillator strips with the WLS fiber. . . . . . . . . . . . . 41

4.7 Scintillator strips in a module. . . . . . . . . . . . . . . . . . 42

4.8 Modules in a plane. . . . . . . . . . . . . . . . . . . . 44

4.9 Lifting a finished plane. . . . . . . . . . . . . . . . . . 44

4.10 A MUX box which typically contains three PMTs. . . . . . . . . . 46

4.11 A strongback carrying a completed plane. . . . . . . . . . . . 46

4.12 The Coil Collars making up the Coil Hole. . . . . . . . . . . . . . . . 48

4.13 Insertion of the magnetic coil tube through a Supermodule. . . . . . 49

4.14 A Far Detector Front End Electronics (FEE) board. . . . . . . . . . 49

4.15 The Far Detector DAQ readout chain. . . . . . . . . . . . . 51

4.16 A more vertical view of the Far Detector DAQ readout chain. . . . . 52 
4.17 The demultiplexing concept: on the left is possible strip combinations versus $\mathrm{Z}$ ( $\mathrm{U}$ view on top, $\mathrm{V}$ view on bottom). Note that each possible track has two colors, light for East, dark for West and these don’t necessarily match up. On the right, only the matched up East and West possibilities are plotted as the correct demultiplexed track. . . 56

4.18 A track in x,y space translated into Hough space (D. Petyt thesis). . 58

4.19 The track finding efficiency versus event length for MC for several different Hough space bin sizes (D. Petyt thesis). . . . . . . . . . 59

4.20 The Far Detector Hall, referenced to the surface. Inset is rotated about y-axis to show face as seen from Northern hall entrance. . . .

4.21 The Far Detector azimuth and zenith coordinates, as defined by the direction cosines of the track. Direction cosines are the components of the track's initial vector in the three axes. . . . . . . . . .

4.22 The left image is that of steel resting on supports, the center of plane is nominally set to the center of the xy-axes. The right image is that of scintillator modules affixed to a steel plane, the center of which is nominally set to the same center as the steel plane. Ultimately, the scintillator plane may be rotated and/or offset from the center of the

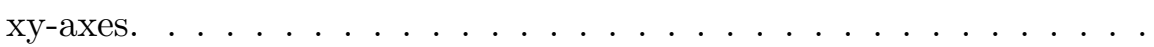

4.23 The left image is of scintillator modules on a plane, and the right is of scintillator strips within a module, each of which has a transverse position in the database to the strip's center. . . . . . . . .

4.24 A low energy cosmic muon track with impact parameter of 2 meters in the MINOS Far Detector. . . . . . . . . . . . . . 67

4.25 A high energy, straight muon track in the MINOS Far Detector. . . 68 
4.26 A low energy muon track in the MINOS Far Detector traversing the coil hole. . . . . . . . . . . . . . . . . . . . . 69

4.27 A low energy, stopping muon track in the MINOS Far Detector. . . . 70

5.1 The efficiency $\epsilon$ of the analysis cuts minus the QPID cut for the MINOS Far Detector as a function of azimuth and zenith. . . . . . 76

5.2 The efficiency $\epsilon$ of the analysis cuts plus the QPID cut for the MINOS Far Detector as a function of azimuth and zenith. . . . . . . . . 76

5.3 The calculated projected area of the MINOS Far Detector in $\mathrm{cm}^{2}$ as a function of azimuth and zenith. . . . . . . . . . . 77

5.4 The MINOS Far Detector's Monte Carlo acceptance in $\mathrm{cm}^{2} \mathrm{sr}$ of muons passing all cuts except the QPID cut as a function of azimuth

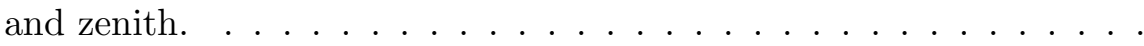

5.5 The MINOS Far Detector's Monte Carlo acceptance in $\mathrm{cm}^{2} s r$ of muons passing all analysis cuts including the QPID cut as a function of azimuth and zenith. . . . . . . . . . . . . 78

5.6 The ratio of the efficiency of positive over negative muon reconstruction in the Forward Field MC, as a function of azimuth and zenith. Efficiency at angles close to perpendicular to z-axis prefers positives for North-going muons and negatives for South-going muons. . . . .

5.7 The ratio of the efficiency of positive over negative muon reconstruction for Reverse Field MC, as a function of azimuth and zenith. Efficiency at angles close to perpendicular to z-axis prefers negatives for North-going muons and positives for South-going muons. . . . . . . 
5.8 The ratio of efficiency of positive over negative muon reconstruction, after summing forward field and reverse field MC sets, as a function of azimuth and zenith. Efficiency is no longer biased to either charge due to the summing procedure. . . . . . . . . . . . .

5.9 The muon data sample from July 2003 through April 2005 as a function of azimuth and zenith. . . . . . . . . . .

5.10 The intensity of incident (calculated without QPID cut) muons at the MINOS Far Detector as a function of azimuth and zenith. See Figure 2.7 to compare intensity near vertical $\approx 1.2 \times 10^{-7} \mathrm{~cm}^{-2} \mathrm{~s}^{-1} \mathrm{sr}^{-1} .81$

5.11 The charge ratio of muon tracks passing cuts as a function of azimuth, Forward field data is solid circles, Reverse field data is open circles. .

5.12 The charge ratio of muon tracks passing cuts as a function of zenith.

5.13 The charge ratio as a function of "detector azimuth" for Forward field data (solid) and Reverse field data (dashed). . . . . . . . . .

5.14 Map of rock traversed by muons in bins of azimuth and zenith, in M.W.E. as viewed from the center of the Hall floor underneath the Soudan 2 detector. . . . . . . . . . . . . .

5.15 Surface elevation above the Soudan 2 detector, centered about the detector's coordinates. . . . . . . . . . . . . . . 88

5.16 Map of rock density in bins of azimuth and zenith, in M.W.E. as viewed from the center of the Soudan 2 Hall floor. . . . . . . . . .

5.17 Map of rock traversed by muons in bins of azimuth and zenith, in M.W.E. as viewed from the center of the MINOS detector. . . . . . .

5.18 The Magnetic Field seen by a muon's perpendicular component (in the xy-plane) as it traverses the detector for impact parameter b. . . 
6.1 Charge Ratio as a function of the azimuth in MINOS Far Detector, (a) is Forward field data, (b) is Monte Carlo and (c) is Data divided by $\mathrm{MC}$, from $[7] . \ldots \ldots \ldots \ldots \ldots \ldots$

6.2 Charge Ratio as a function of the zenith in MINOS Far Detector, (a) is Forward field data, (b) is Monte Carlo and (c) is Data divided by MC, from $[7] . \ldots \ldots \ldots \ldots$

6.3 Charge Ratio as a function of the reconstructed momentum in MINOS Far Detector, (a) is Forward field data, (b) is Monte Carlo and (c) is Data divided by MC, from $[7] . \ldots \ldots \ldots \ldots$

6.4 Charge Ratio versus azimuth, no QPID cut, MC is a solid line, data is filled circles. . . . . . . . . . . . . . . .

6.5 The Charge Ratio versus the "QPID cut": $(q / p) / \sigma(q / p)$. The Data is points with error bars while the $\mathrm{MC}$ is the solid line. Note: $\mathrm{MC}$ has an input charge ratio of $1.25 \ldots \ldots \ldots \ldots$

6.6 The charge ratio versus azimuth, Forward field Data before QPID cut is in filled circles, same data after the QPID cut is displayed in open

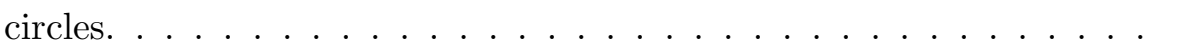

6.7 Summed charge ratio versus QPID for a range of $|\mathbf{d} \operatorname{cosz}|$. The point at which charge ratio levels off indicating background is removed, is a function of dcosz. . . . . . . . . . . . . . . . . 103

6.8 Two charge ratio data sets versus azimuth, Forward is in dark circles, Reverse is in open circles. . . . . . . . . . . . . . . . . . . . 104

6.9 Normalized and summed Forward and Reverse data sets, data is in dark circles, MC is the solid line and has been scaled to have the same charge ratio as the data $(1.347) \ldots \ldots \ldots$. . . . . . 106

6.10 The two-week SM1 “Alignment Data Set's" charge ratio versus azimuth.106 
6.11 The two-week SM2 “Alignment Data Set's" charge ratio versus azimuth.107

6.12 Sagitta positions in the detector for SuperModule 2 field off data. . . 107

6.13 The charge ratio as a function of detector azimuth for the Toy Monte Carlo. .......................... 11

6.14 A Map of the Modified Magnetic Field used with the Toy Monte Carlo in Tesla. . . . . . . . . . . . . . . . . . . . . .

6.15 The charge ratio as a function of detector azimuth for the Toy Monte Carlo, using the modified magnetic field. The sine wave variation in the charge ratio of Figure 5.13 is not present. . . . . . . . . .

7.1 The Charge Ratio as a function of azimuth for summing the same data with both field configurations. . . . . . . . . . . . 120

7.2 The Charge Ratio as a function of azimuth for summed data sets, Data is with error bars, Monte Carlo is continuous line wherein the MC charge ratio has been scaled to a charge ratio of 1.35 for visual comparison with data. . . . . . . . . . . . . . .

7.3 The Charge Ratio as a function of zenith for summed data sets, Data is with error bars, Monte Carlo is continuous line wherein the MC charge ratio has been scaled to a charge ratio of 1.35 for visual comparison with data. $\ldots \ldots \ldots \ldots \ldots \ldots$

7.4 The Charge Ratio as a function of the angle in the XY plane, about the Z-axis, for summed data sets, Data is with error bars, Monte Carlo is continuous line wherein the MC charge ratio has been scaled to a charge ratio of 1.35 for visual comparison with data. . . . . . . 
7.5 The Charge Ratio as a function of the angle from the Z-axis that the muon is travelling towards, for summed data sets, Data is with error bars, Monte Carlo is continuous line wherein the $\mathrm{MC}$ charge ratio has been scaled to a charge ratio of 1.35 for visual comparison with data. Not expected to be flat. . . . . . . . . . . . . . . . . . . . . 123

7.6 Plot of Figure 7.5, after reweighting to contain the same charge ratio dependence on slant depth. MC is solid line, data is filled circles. . . 125

7.7 Plot of charge ratio versus slant depth, after reweighting by dcosz to contain the same charge ratio dependence on dcosz as in data. MC is solid line, data is filled circles. . . . . . . . . . . . . . . . . 125

7.8 The Charge Ratio as a function of slant depth after the summing procedure. . . . . . . . . . . . . . . . . 126

7.9 The Charge Ratio as a function of surface muon energy after the summing procedure. . . . . . . . . . . . . . . 126

8.1 The Charge Ratio fit versus slant depth for Sample 1. . . . . . . . . 130

8.2 The Charge Ratio fit versus slant depth for Sample 2. . . . . . . . . 130

8.3 The integrated Charge Ratio as a function of cuts on plane number, track length and track vertex dcosz. . . . . . . . . . . . . . 132 


\section{Chapter 1}

\section{Introduction}

Cosmic Rays are extraterrestrial atomic nuclei that span an energy range from a few $\mathrm{MeV}$ to beyond $10^{20} \mathrm{eV}$. The differential spectrum as a function of energy over most of this range roughly follows $\frac{d N}{d E} \propto E^{-\gamma}$, where $\gamma$ is between 2.7 and 3.0. Upon encountering the Earth's atmosphere, these nuclei interact in p,n-Air collisions to produce a shower of secondary particles (pions, kaons and other hadronic particles), some of which then decay into muons and muon-type neutrinos as shown in Figure 1.1 .

The secondary particles either interact further with the atmosphere or rapidly decay into muons and neutrinos as $\pi^{+} \rightarrow \mu^{+}+\nu_{\mu}$ and $K^{+} \rightarrow \mu^{+}+\nu_{\mu}$ and their associated anti-particles decay into $\mu^{-}$and $\bar{\nu}_{\mu}$. The charge ratio of the resultant muons is then dependent upon the $\pi / K$ hadronic production ratio, the proton to neutron primary composition ratio, the primary energy spectrum and atmospheric conditions such as pressure and local magnetic field.

At the surface of the Earth, the secondaries that have not yet decayed interact, leaving muons and neutrinos as the dominant sub-surface component. Neutrinos will penetrate the entire Earth without significant reduction of flux, while muons lose energy at a known energy dependent rate of several hundred $\mathrm{MeV}$ per meter in rock until the energy is low enough for decay or atomic capture in the rock 


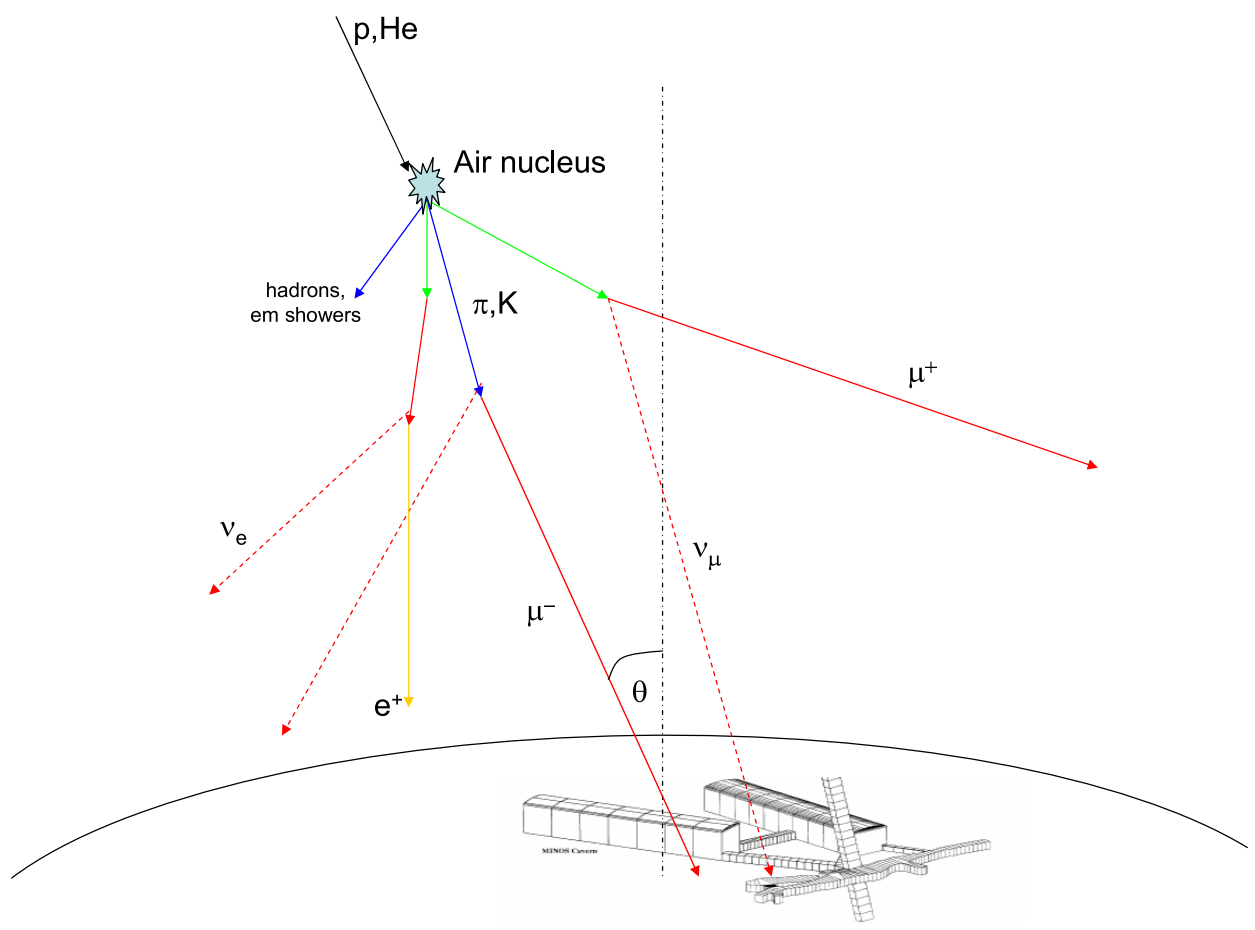

Figure 1.1: A cartoon of a cosmic ray air shower, with remnant particles extending into the Earth over the Soudan Underground Laboratory. 


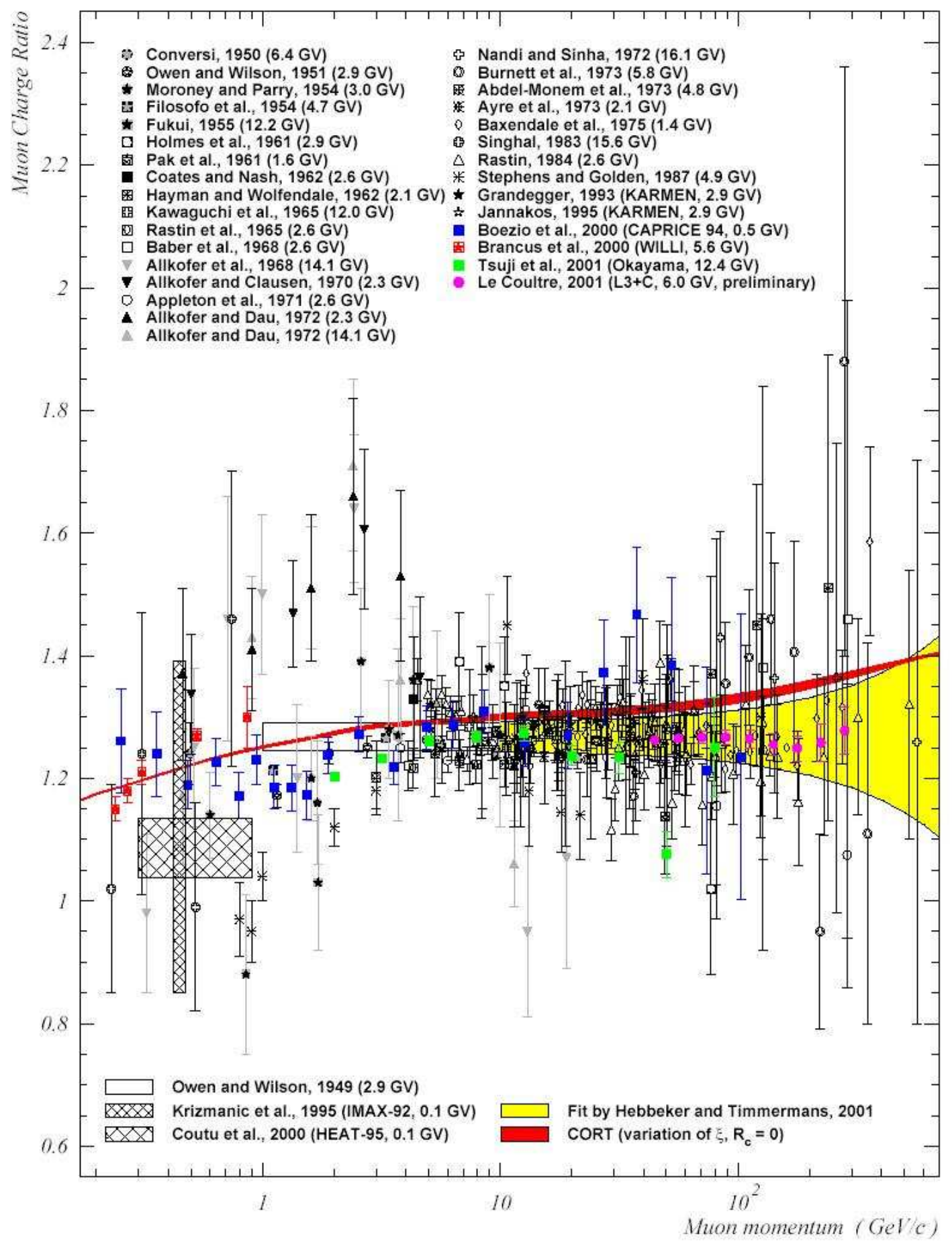

Figure 1.2: Compilation of recent experimental results, with world fit overlain and CORT predictions in red line (from Naumov). 
(and subsequent decay) to occur. The result of this muon energy loss is that an overburden of many tens, hundreds or even thousands of meters of water equivalent (MWE) translates to an effective lower energy bound of original (surface) muon energy. The MINOS Far Detector is a deep underground magnetized steel and scintillator detector, so it is well suited to performing a precision measurement of the charge ratio of cosmic ray muons over the surface muon energy range $700 \mathrm{GeV}$ to about $5 \mathrm{TeV}$.

Recent work on atmospheric muon calculations by Naumov [8] using updated primary compositions and hadronic production data with the software code CORT (Cosmic Origin Radiation Transport) predicts an increase in charge ratio as energy increases. The CORT calculation of the charge ratio over a wide energy range, along with an encyclopedic compilation by Naumov of many of the muon charge ratio experiments is shown in Figure 1.2. MINOS has an overburden that increases roughly as $\frac{1}{\cos (z e n i t h)}$, and most of the sample of measured muons lie in the range from 2000 MWE up to around 8000 MWE and thus could explore this energy dependence of the charge ratio.

This thesis presents the measurement of the charge ratio with the MINOS Far Detector, and studies potential systematics associated with the MINOS data analysis. Chapter 2 sets the stage by summarizing cosmic ray physics in the atmosphere and muon behavior in the rock. Chapter 3 covers previous measurements of the muon intensity and charge ratio over a wide energy range by other experiments. Chapter 4 describes the MINOS Far Detector and the software reconstruction of the events. The response of the detector to muons is explored in Chapter 5. Chapter 6 discusses the measurement of the charge ratio, problems that were encountered in the measurement and the work done to solve them. Chapter 7 presents a solution to the problem and gives the charge ratio in terms of slant depth and extrapolated 


\begin{tabular}{|l|c|}
\hline Symbol & Meaning \\
\hline \hline$\phi$ & Azimuth \\
$\theta$ & Zenith \\
$\epsilon$ & efficiency \\
$\sigma$ & sigma \\
$\mathbf{b}$ & impact parameter \\
$\mathbf{d c o s x}$ & direction cosine-x of track \\
$\mathbf{d c o s y}$ & direction cosine-y of track \\
$\mathbf{d c o s z}$ & direction cosine-z of track \\
$\chi^{2}$ & Chi-Squared \\
\hline
\end{tabular}

Table 1.1: Symbols Used

surface muon energy. Chapter 8 gives an analysis of the remaining systematic biases that may be present and how to improve the measurement. 


\section{Chapter 2}

\section{Cosmic Ray Muons}

Muons have a relatively long lifetime and fairly small interaction cross section, so they comprise a significant fraction of the cosmic ray flux at surface elevation. They penetrate deeply underground and lose energy in the rock at a well understood, energy dependent rate. This chapter discusses the origin and transport of cosmic ray muons and the calculations of the flux and charge ratio.

\subsection{Cosmic Ray Muon Origin}

\subsubsection{Cosmic Ray Primaries}

Cosmic muons detected by experiments such as MINOS are not cosmic in origin but are the detectable remnants of a "cosmic ray shower" of secondary particles generated by a cosmic ray primary particle interacting in the upper atmosphere. The primaries (at the relevant energies) are composed of about $90 \%$ hydrogen nuclei, $9 \%$ helium, and $1 \%$ heavier nuclei [9]. The proportions of this composition are fairly constant with energy up to the "knee" of the primary spectrum, around $3 \times 10^{15} \mathrm{eV}$. The energies of primaries range from $10^{7} \mathrm{eV}$ all the way up to $10^{20} \mathrm{eV}$ and above. Almost the entire spectrum can be described by an inverse power law in energy where the differential flux per nucleon is given by Equation 2.1: 


$$
\frac{d N}{d E} \approx E^{-\alpha}
$$

This approximation is valid from an energy of $20 \mathrm{GeV}$ up to about $10^{15} \mathrm{eV}$ with $\alpha \approx 2.7$. At around $3 \times 10^{15} \mathrm{eV}$, or the "knee" of the spectrum, it steepens to about $\alpha \approx 3.0$ until about $10^{19} \mathrm{eV}$. This is thought to be due to the changing primary spectrum to incorporate higher atomic number nuclei. Above $10^{19} \mathrm{eV}$, named the "ankle" of the spectrum, the spectrum becomes less steep again. Above

these ultrahigh energies, near $10^{20} \mathrm{eV}$, the spectrum is expected to fall off more rapidly due to interaction with the cosmic microwave background radiation, known as the GZK cutoff. At the lower end of this scale, cosmic rays with energies below $10 \mathrm{GeV}$ are affected by the solar wind and terrestrial geomagnetic cutoffs. The behavior of the spectrum of the various nuclei can be seen in Figure 2.1, where five groups of nuclei from protons up through iron nuclei are plotted. The heavier nuclei are expected to fall off more slowly, leading to a general increase in the proportion of neutrons versus protons. The integral over energy gives the nucleon rate, which then follows $\alpha+1$.

\subsubsection{Primary Interaction with Atmosphere}

Incoming cosmic ray nucleons enter the atmosphere and travel on average one interaction length before interacting. The mean free path, or the interaction length for a cosmic ray nucleon in air, is given by Equation 2.2:

$$
\Lambda_{N}=\frac{\rho}{\rho_{N} \sigma_{N}^{a i r}}=\frac{A m_{p}}{\sigma_{N}^{a i r}}
$$

where $\rho=$ density of air, $\rho_{N}=$ nucleon density, $\sigma_{N}^{\text {air }}=$ cross section of nucleon in air, $A=$ atomic number of air nucleus, and $m_{p}=$ mass proton. For nucleons in the 


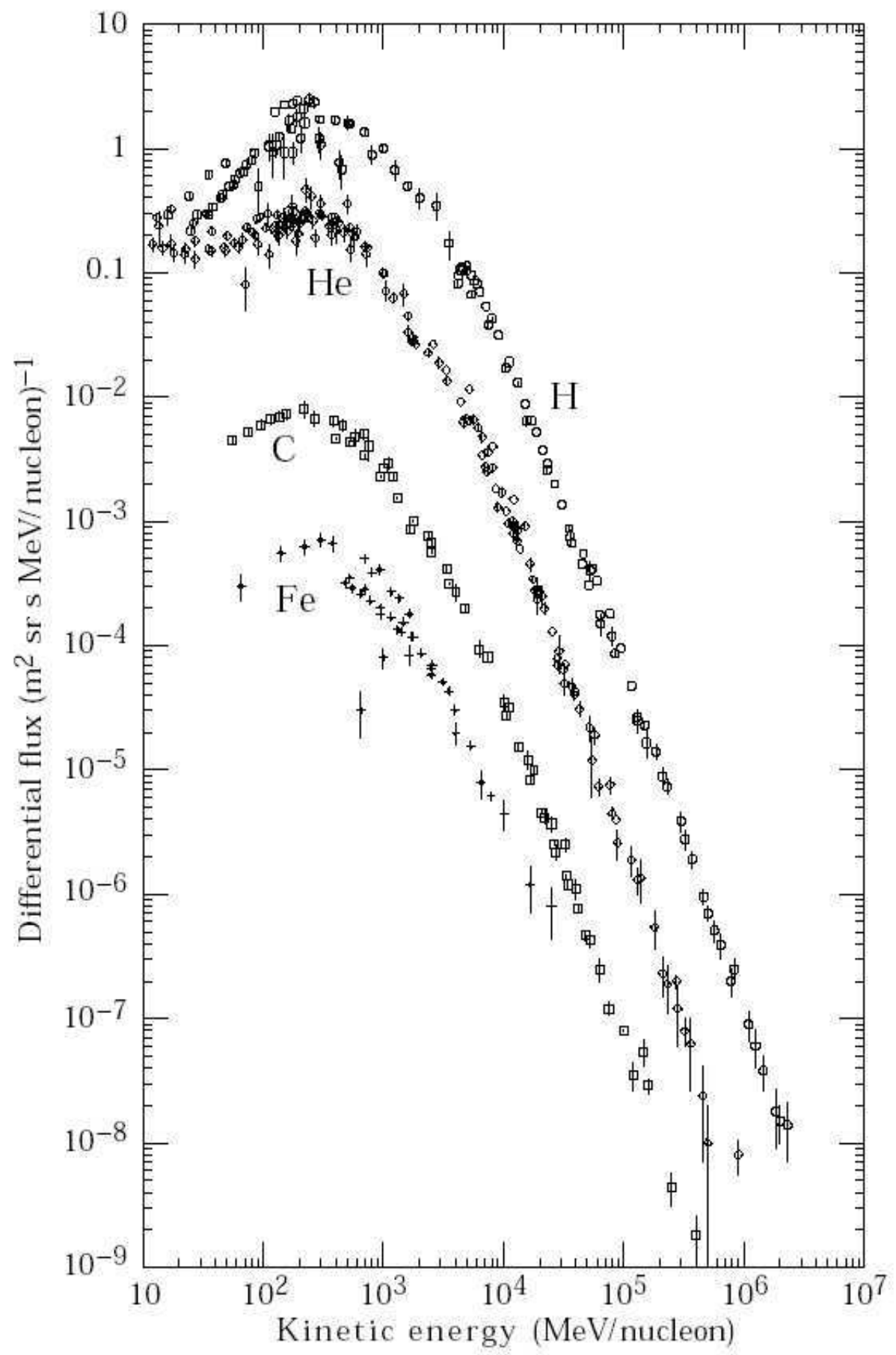

Figure 2.1: Caption and Figure taken from [1], data points from [2]. 
$\mathrm{TeV}$ range, $\sigma_{N} \approx 300 \mathrm{mb}$. Taking A to be $16, \Lambda_{N} \approx 80 \mathrm{~g} / \mathrm{cm}^{2}$, although $\Lambda$ is energy dependent. The vertical intensity of the nucleons can be expressed:

$$
I_{N}(E, X) \approx I_{N}(E, 0) e^{(-X / \Lambda)}
$$

The depth in the atmosphere, called "slant depth", is given by X, in total $\mathrm{g} / \mathrm{cm}^{2}$ traversed since the "top" of the atmosphere, which is typically 60 kilometers above sea level. Since the total atmospheric depth is around $1000 \mathrm{~g} / \mathrm{cm}^{2}$, the primary nucleons will not survive to the surface but will instead interact with the air and create pions and kaons.

\subsubsection{Hadron Production}

\subsubsection{Pions}

In a typical proton-proton collision, pions are produced like $p+p \rightarrow p+p+$ $\pi^{+}+\pi^{+}+\pi-+$ etc, with the multiplicity dependent primarily upon the inelasticity of the collision, denoted as $\kappa$. This parameter determines the proportion of initial nucleon energy available for the interaction and has been measured in accelerator experiments for a wide range of energies. Since the protons are positively charged, and the proportion of anti-matter in the cosmic rays is negligible, there are more positive than negative pions produced. In air, there are roughly an equal number of neutrons as protons available, and in the cosmic rays, the proportion that are neutrons is roughly $18 \%$. The neutron-neutron collisions produce just as many positive pions as negatives and so pull the pion charge ratio towards 1.0. So only about $9 \%$ (50\% of $18 \%$ ) of the collisions produce equal numbers of $\pi^{+}$and $\pi^{-}$, while the other $91 \%$ contribute an excess of $\pi^{+}$. 


\subsubsection{Kaons}

Kaons and $\Lambda$ 's on the other hand, require strangeness production. The kaon is more likely to be positive regardless of whether neutrons or protons were involved in the collision. A hadronic collision with enough energy for creating a strangeantistrange pair will preferentially put the strange quark in a baryon with two other quarks, since the collision starts with quarks rather than antiquarks. The antistrange would then likely either be paired with a down quark to make a neutral kaon or an up quark to make a positive kaon. This will tend to be true up to high enough energies where enough anti-matter can be created to make an antibaryon. Several reaction channels for proton-proton collisions are shown in Figure 2.2. Neutron-proton collisions have similar reaction channels, although the resultant meson charges produced are $1 / 3$ rd the proton-proton collisions (more neutral kaon possibilities with the extra down quark) as in Figure 2.3.

\section{$2.2 \quad$ Charge Ratio}

Using available data and some simple assumptions, Naumov [10] has obtained an estimate of the charge ratio, which is repeated here. The first assumption is that cosmic muons come from pion decay alone. The cross sections for pion production in nucleon collisions satisfy the relations:

$$
\frac{d \sigma_{p \rightarrow \pi^{+}}}{d E_{\pi}}=\frac{d \sigma_{n \rightarrow \pi^{-}}}{d E_{\pi}} \text {, and } \frac{d \sigma_{p \rightarrow \pi^{-}}}{d E_{\pi}}=\frac{d \sigma_{n \rightarrow \pi^{+}}}{d E_{\pi}}
$$

Since around $9 \%$ of the primaries are alpha particles, there are neutrons in the primary mix along with the more numerous protons. The primary composition data from Figure 2.1 suggest a slow decrease in $R_{p / n}$ with energy, as the composition becomes heavier. Approximating using only hydrogen and helium nuclei, $\phi_{p}=$ 

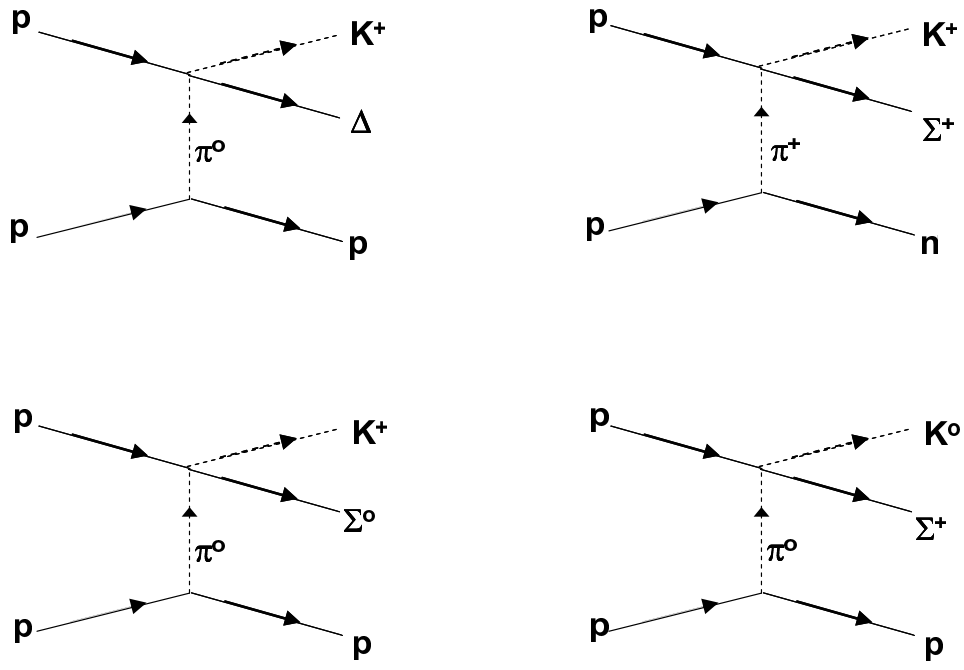

Figure 2.2: Kaon production diagrams for $p p \rightarrow p, n Y K$ reaction channels.
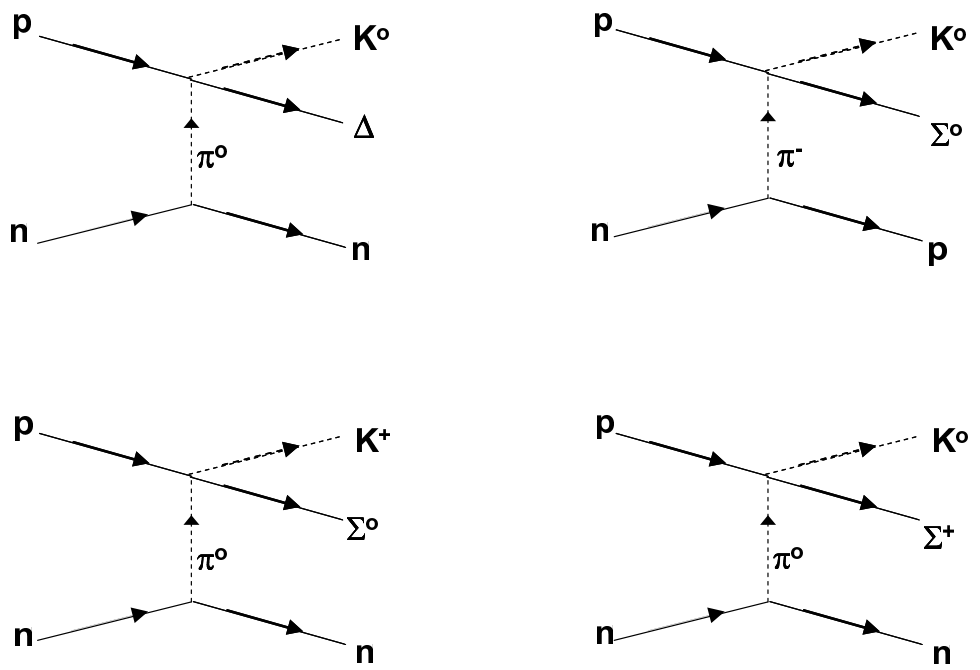

Figure 2.3: Kaon production diagrams for $p n \rightarrow p, n Y K$ reaction channels. 
$\phi_{H}+2 \phi_{H e}$ and $\phi_{n}=2 \phi_{H e}$, so $\phi_{p}=\left(\phi_{H} /\left(2 \phi_{H e}\right)\right)+1 \approx 5.5$. As the composition includes more of the heavier elements, this will decrease.

With the assumption that pions and only pions decay into the muons, the charge ratio of the muons is then given by Equation 2.5:

$$
r=\frac{N_{\pi^{+}}}{N_{\pi^{-}}}=\frac{N_{\mu^{+}}}{N_{\mu^{-}}}=\frac{\left(1+\alpha_{\pi}\right) R+1}{R+1+\alpha_{\pi}},
$$

The $\pi^{+}$production excess in p-Air collisions is given by $\alpha \approx 0.35$. Using these numbers and the pion only approximation, the result is $r \approx 1.23$. At the energies MINOS is sensitive to, two-body kaon decay must be taken into account. Equation 2.5 can be rewritten to account for the kaons in Equation 2.6:

$$
r=\frac{\left(1+\alpha_{\pi}+K^{+} / \pi_{+}\right) R+\left(1+K^{+} / \pi^{+}\right)}{\left(1+K^{-} / \pi^{-}\right) R+\left(1+\alpha_{\pi}+K^{-} / \pi^{-}\right)}
$$

where $K^{+} / \pi^{+}$and $K^{-} / \pi^{-}$are kaon to pion production ratios in p,n-Air collisions. These production ratios are not available from accelerator data in the MINOS energy range, but data [11] using $200 \mathrm{GeV}$ mesons produced from $450 \mathrm{GeV}$ proton collisions give $K^{+} / \pi^{+}=0.15$ and $K^{-} / \pi^{-}=0.05$, with the negative kaon deficit due to the strangeness production mentioned in the previous section on kaons. With the addition of the kaon contribution, the charge ratio prediction rises to $r=1.31$.

\subsubsection{Mitigating Factors}

The proton to neutron ratio does not remain constant as a function of energy, and is expected to decrease, especially near the knee of the primary spectrum. Neutron-Air collisions produce a smaller excess of positive pions than proton-Air collisions. As can be seen in the production possibilities in Diagrams 2.2 and 2.3, the kaon charge ratio is even more sensitive to the proton-neutron ratio. 
Another factor is the hadronic production ratios. The positive versus negative

pion and kaon production ratios are expected to decrease with energy [10] slowly and logarithmically. Contribution from semileptonic decay of charged and neutral kaons increases as energy increases and contribution from decay of charmed hadrons becomes important at even higher energies.

\subsection{Cosmic Ray Muons}

\subsubsection{Energy Spectrum}

Over the muon surface energy range relevant to MINOS, the primary cosmic ray creating that particular muon had an average energy of approximately 10 times the muon's surface energy [9]. At MINOS's depth, about $90 \%$ of the muons with a measureable momentum derive from primaries in the range from $5 \mathrm{TeV}-50 \mathrm{TeV}$ $[3]$.

Most muons are produced high in the atmosphere, around $15 \mathrm{~km}$ or higher. Approximately $2 \mathrm{GeV}$ of the energy is lost by ionization in the atmosphere, so for muons with energies greater than $500 \mathrm{GeV}$, the surface energy can be considered the production energy. Folding the production spectrum of the pions and kaons together, and taking account of energy loss and decay in the atmosphere, the energies of muons at the surface follow Equation 2.7, known as the Gaisser parameterization [9] from the Particle Data Book [1] section on Cosmic Ray Muons (section written by Gaisser and Stanev). This parameterization is valid when muon decay is negligible $\left(E_{\mu}>100 / \cos \theta \mathrm{GeV}\right)$ and curvature of the Earth can be neglected [1].

$$
\frac{d N_{\mu}}{d E_{\mu}} \approx \frac{0.14 E_{\mu}^{-2.7}}{c m^{2} s s r G e V} \times\left(\frac{1}{1+\frac{1.1 E_{\mu} \cos \theta}{115 G e V}}+\frac{0.054}{1+\frac{1.1 E_{\mu} \cos \theta}{850 G e V}}\right)
$$

This equation accounts for the pions in the first term, and the kaons in the sec- 
ond, while it neglects the charm contribution entirely, which is considered negligible in this context. Data points are shown in Figure 2.4.

\subsubsection{Energy Loss}

Muons lose energy by radiative processes (bremsstrahlung, pair production and photonuclear interactions) and ionization in the rock overburden. The energy loss can be calculated to determine if a muon at some original energy would survive the traversal of some amount of rock, and these survival probability curves for muons in Soudan rock are shown in Figure 2.5 from Sue Kasahara's thesis [3]. Muon transmission turns on very quickly, and because of the rapidly falling spectrum of muon flux, most muons observed at a particular slant depth will come from just above the transmission turn-on point, especially if the measured muons are restricted to energies at the detector of below $150 \mathrm{GeV}$. Slant depth of some overburden, whether water, Earth, ice or air is commonly given in units of meters of water equivalents (M.W.E.), or $\mathrm{kg} / \mathrm{m}^{2}$.

The muon energy loss as a function of matter traversed is given by the BetheBloch formula in Equation 2.8 and parameterized for typical muons in matter in Equation 2.9 [1].

$$
\begin{aligned}
\frac{d E}{d x} & =\frac{2 \pi n e^{4}}{m c^{2} \beta^{2}}\left[\ln \left(\frac{2 m p^{2} W}{I^{2} \mu^{2}}\right)-2 \beta^{2}-\delta-U\right] \\
& =-a-b E
\end{aligned}
$$

Equation 2.9 gives energy lost per $\mathrm{g} / \mathrm{cm}^{2}$. The variable a incorporates ionization losses and the radiative processes are incorporated by $\mathbf{b}$. The factors $\mathbf{a}$ and $\mathbf{b}$ are energy dependent and were parameterized for standard rock as $a=$ $2.04+0.0782 \ln \left(E_{\mu}\right) M e V g^{-1} \mathrm{~cm}^{2}$ and $b=1.92+0.278 \ln \left(E_{\mu}\right) g^{-1} \mathrm{~cm}^{2}$ (using 


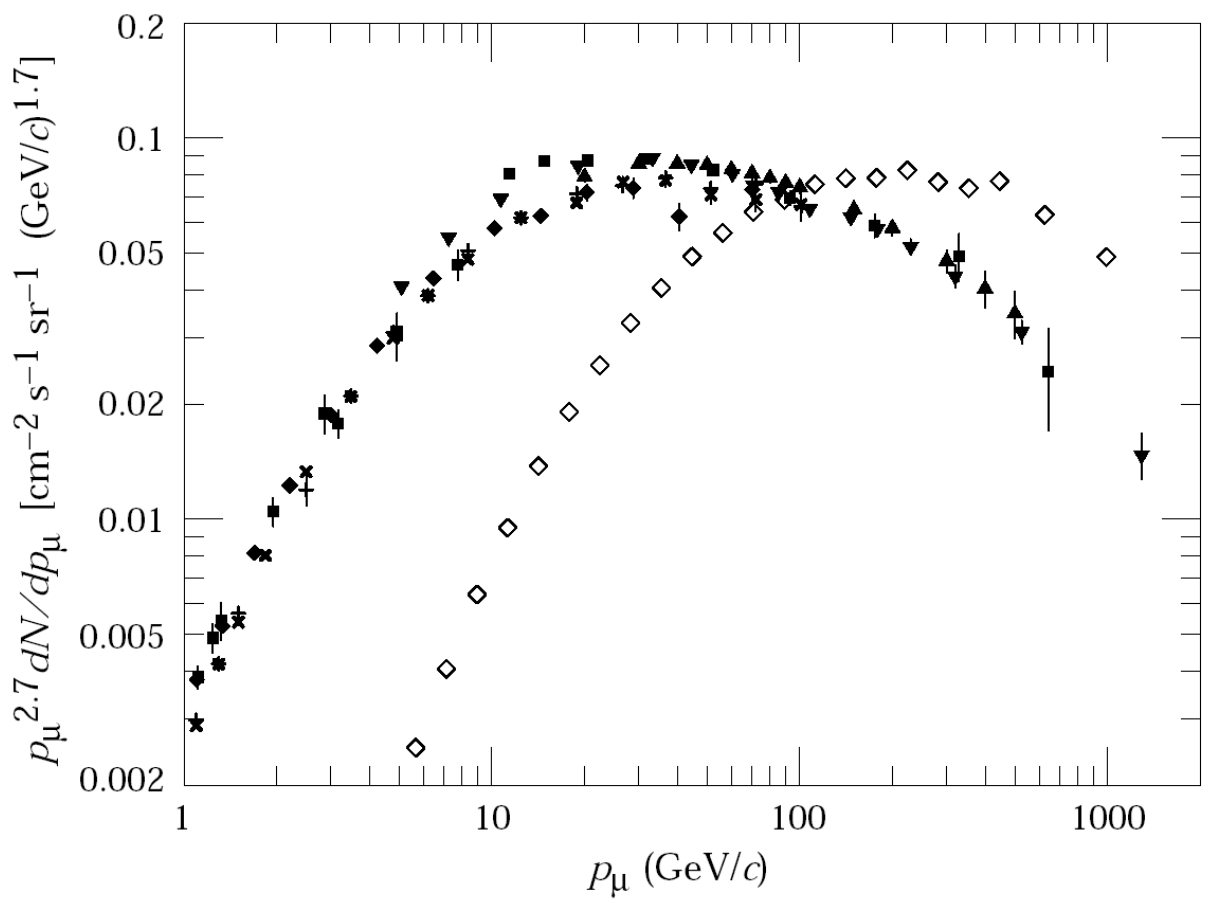

Figure 2.4: Muon rates, taken from [1]. 


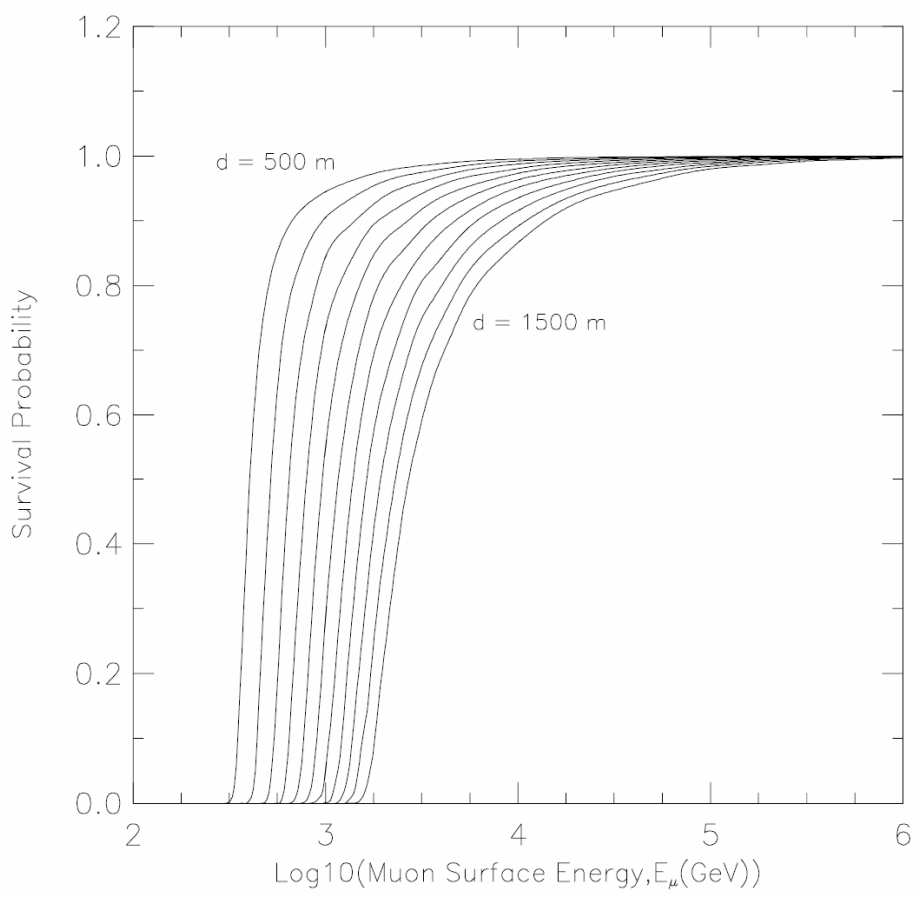

Figure 2.5: Muon survival curves as function of original energy for various slant depths of rock [3]. 
Table 24.2 from PDG)[1]. These are dependent upon the chemical composition of the matter they refer to, in particular $\mathbf{a}$ is roughly proportional to $Z / A$, while $\mathbf{b}$ is roughly proportional to $Z^{2} / A$.

$$
\begin{gathered}
R(X)=\int_{0}^{d} d x=\int_{E_{o}}^{0} \frac{d E_{\mu}}{-a-b E_{\mu}}=\frac{1}{b} \ln \left(1+\frac{E_{o}}{a / b}\right) \\
E(X)=\left(E_{o}+\frac{a}{b}\right) \times e^{-b X}-\frac{a}{b}
\end{gathered}
$$

The solution of Equation 2.9 is given in Equation 2.10 and can be used to get approximate energies relevant to an underground experiment. In particular, a threshold energy of $750 \mathrm{GeV}$ is required for an overburden of 2,000 M.W.E. while an overburden of 6,000 M.W.E. requires a threshold energy of 8,300 GeV. The method used to estimate the surface energy of muons with a particular azimuth and zenith angle is as follows:

(1) a map of the rock slant depth above the detector was used to find the slant depth $\mathrm{X}$ for this bin,

(2) Equation 2.10 was used to find the threshold energy needed to traverse this slant depth,

(3) the threshold energy was used to get $\mathbf{a}$ and $\mathbf{b}$,

(4) an assumption of a final energy at MINOS of $\mathrm{E}(\mathrm{X})=30 \mathrm{GeV}$ is put into Equation 2.11, which is then solved for $E_{o}$.

The final energy at MINOS was assumed $30 \mathrm{GeV}$ since the measurement is only sensitive to muons with a final energy below $100 \mathrm{GeV}$. Muons lose around 2/3rds $\mathrm{GeV}$ per meter in rock, so a $30 \mathrm{GeV}$ muon will have a range of approximately 45 meters. The average distance to surface is about 1000 meters, so $45 \mathrm{~m} / 1000 \mathrm{~m} \approx$ 


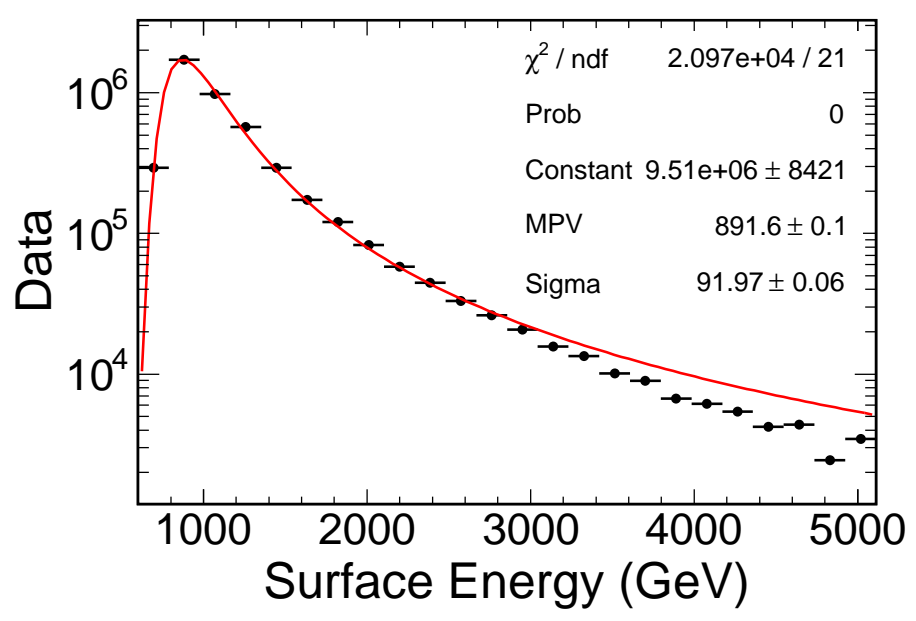

Figure 2.6: Histogram of surface energy for measured (low energy) muons based on overburden and Equation 2.11.

$5 \%$ is the estimate of the accuracy of this method. Any further work on this should involve a full muon propagation simulation study. A simulation study was done to investigate the spectrum observed underground which will be mentioned later in this chapter, but the charge separation of the muons is only possible for approximately $1 / 5^{\text {th }}$ of the total muon sample, so the simulation's results are not applicable to determining the surface energy of muons.

\subsubsection{Soudan Rock}

Standard rock has $\frac{Z}{A}=0.5$ and $\frac{Z^{2}}{A}=5.5$ and a density of $\rho=2.65 \mathrm{gm} / \mathrm{cm}^{3}$. The rock around the Soudan site was examined and found to have the following properties: $\frac{Z}{A}=0.496, \frac{Z^{2}}{A}=5.98[12]$ and $\rho=2.80 \mathrm{~g} / \mathrm{cm}^{3}$. Just North of the mine, the average rock density was found to be higher by as much as $10 \%$ and there appears to be a band of about $10 \%$ higher density ore above the detector running East-West along the ridge. This was determined using the Soudan 2 detector and its muon sample by Kasahara [3]. A map of the density of the rock above the detector 
will be examined in Chapter 5 .

\subsubsection{Charge Dependence of Energy Loss}

In this thesis, the energy loss of $\mu^{+}$is considered to be equal to that of $\mu^{-}$. There are higher order corrections to the cross sections and hence energy loss that leads to a charge dependence [13], [14]. These should be taken into account in a follow-up analysis.

\subsubsection{Scattering}

The effect of multiple scattering in the rock on the muon direction and position underground has been explored by Soudan 2 collaborator H. Trost [15]. The primary contributions to any deviations are multiple Coulomb scattering and delta ray production, while the radiative processes are small, although occasionally with

very large tails in their effects (catastrophic losses). Various Gaussian approximations were examined and compared with data, with the result that muons passing the minimum overburden of 700 meters of Soudan rock have angular deviations from initial angle of between $2.4 \mathrm{mrad}$ for $10 \mathrm{TeV}$ (energy at surface) muons up to 6.74 mrad for $1 \mathrm{TeV}$ muons. This angle will be compared later with expected curvature of oppositely charged muons in the Earth's magnetic field as a possible source of systematic error in a measurement.

\subsubsection{Propagation Through Rock}

H. Trost has simulated muons passing through 700 meters of Soudan rock to determine the underground muon energy expected at the site [16]. After a comparison with accelerator data and underground experimental data from NUSEX, results were given for muon survival, which I summarize here. Transmission begins around 
$600 \mathrm{GeV}$ and rises sharply with energy. It reaches $50 \%$ near $710 \mathrm{GeV}$ and $90 \%$ at 1.6 TeV. The mean energy of muons at the detector site (underground, after passage of 700 meters rock) is predicted to be $530 \mathrm{GeV}$, with r.m.s. of $280 \mathrm{GeV}$. These same muons have a mean surface energy of $2.10 \mathrm{TeV}$, with an r.m.s. of $2.49 \mathrm{TeV}$. Finally, the spread of the energies is very large, due to the catastrophic nature of radiative losses.

\section{$2.4 \quad$ Expected at MINOS}

\subsubsection{Intensity Expected}

A previous experiment in the Soudan Underground Laboratory, Soudan 2, determined the muon intensity for various zenith angles. The rock map was used to determine average slant depths for particular zenith angles, which is shown in Figure 2.7 as arrows overlain upon the world average data of Crouch et al [17]. The intensity observed at MINOS has been measured and shown to be consistent with the world survey results in the Rebel thesis [7].

This overburden goes roughly as $2000 \mathrm{MWE} / \cos (z e n i t h)$ from 2,000 MWE to 14,000 MWE, where neutrino-induced muons dominate the sample. The rock map will be discussed in more detail later, but suffice it to say that there is significant structure in the rock above MINOS. The rock density varies from 2.70 to 3.05 $\mathrm{g} / \mathrm{cm}^{3}$, and the surface elevation varies from 610 to 710 meters above the center of

the MINOS detector. The density is increased in a band crossing East-West above the detector following known regions of iron deposits, and the 50 meter ridge also extends East-West above the detector. Taken together, these variations lead to variations in the slant depth from the $1 / \cos ($ zenith) approximation of $20 \%$. 


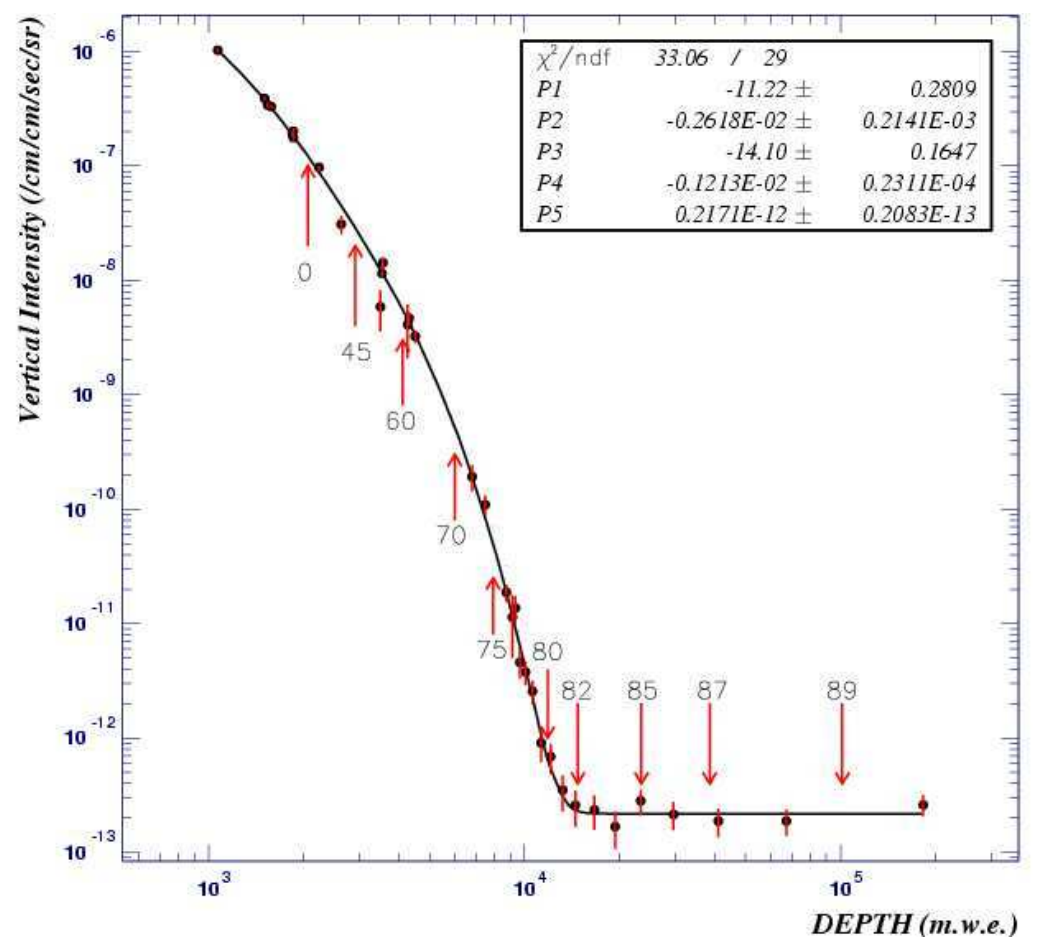

Figure 2.7: Muon intensity versus slant depth, taken from [4]. Slant depths corresponding roughly to particular zenith angles from the Soudan hall are marked and are valid for MINOS site. 


\subsubsection{Charge Ratio Expected}

The surface muon energies explored by MINOS are from $700 \mathrm{GeV}$ to 5000 $\mathrm{GeV}$, as seen in Figure 2.6. Based on the calculated curve [8] shown in Figure 1.2 and private communication with Naumov[10], a charge ratio of $1.4 \pm 0.3$ is expected. 


\section{Chapter 3}

\section{Previous Measurements of the Charge Ratio}

Experiments which measure the muon charge ratio roughly fit into two types: above and below ground (underwater or underice fitting into the same category as below ground). Balloon and surface measurements are sensitive to the lower energy muons that are susceptible to geomagnetic and seasonal effects. Above 10 $\mathrm{GeV}$, the charge ratio of cosmic ray muons is expected to be constant regardless of experimental location, method and time of year, but not necessarily constant as a function of the energy being measured.

A few previous measurements are directly relevant to the MINOS measurement. The CAPRICE balloon experiment, the WILLI muon decay spectrometer, and the $\mathrm{L} 3+\mathrm{C}$ and COSMO-ALEPH measurements are the recent experiments examined below.

\subsection{Surface Measurements}

The compilation of the charge ratio data by Naumov is shown in Figure 3.1 and the spread on measurements can be seen to be a few percent approaching $10 \%$ nearer to MINOS's energy range. The systematic errors quoted by particular measurements is typically far below the spread of measurements for a given energy, indicating the presence of unaccounted systematic errors. 


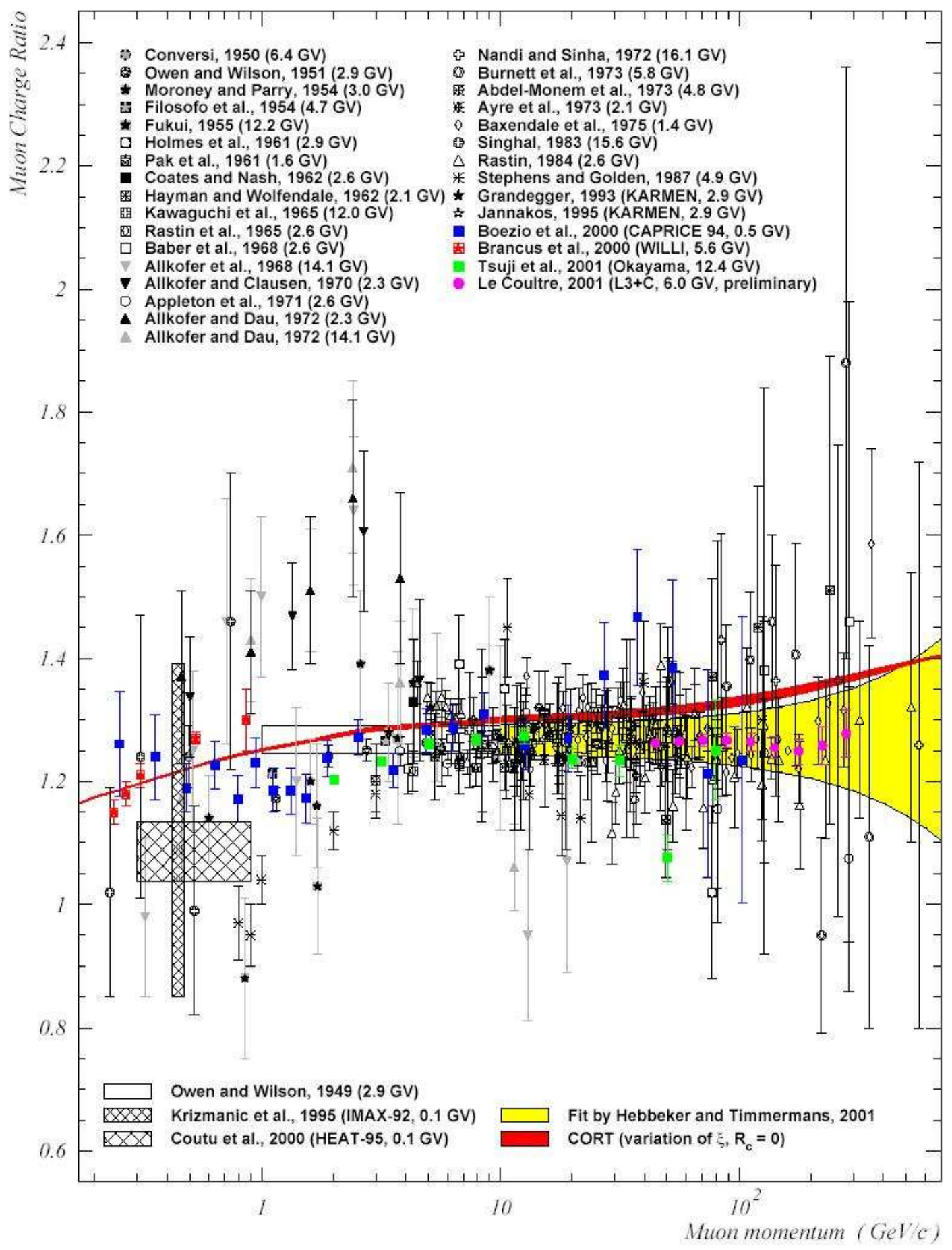

Figure 3.1: Compilation of recent experimental results, with world fit overlain and CORT predictions in red line (from Naumov). 


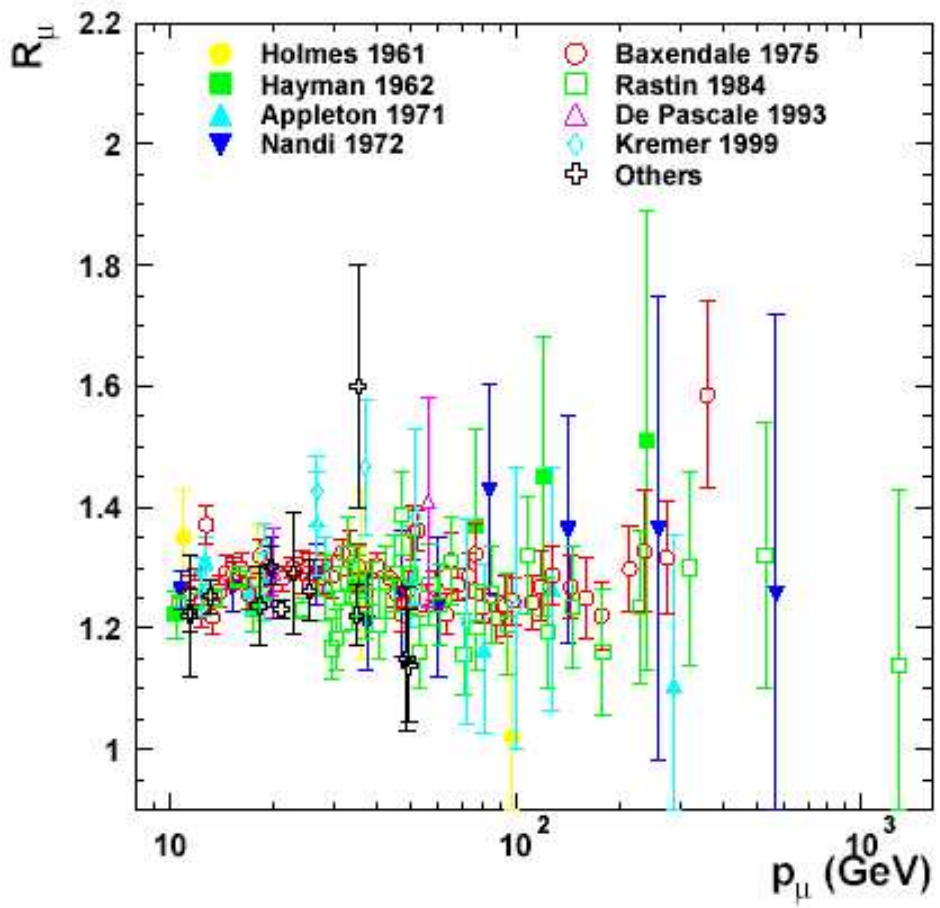

Figure 3.2: Compilation of recent charge ratio measurements, from Hebbeker and Timmermans. 
A "world survey" of previous measurements is shown in Figure 3.2 and is given by Hebbeker and Timmermans [18] as $1.268 \pm 0.008$ (stat.) \pm 0.015 (syst.). This fit assumes a constant charge ratio over the energy range of these measurements, which spans from $10 \mathrm{GeV}$ up to $200 \mathrm{GeV}$. This world survey (which includes data points from Caro et al 1950, Owen et al 1951, Filosofo et al 1954, Pine et al 1959, Holmes et al 1961, Pak et al 1961, Hayman and Wolfendale 1962, Appleton et al 1971, Allkofer and Dau 1972, Nandi et al 1972, Baxendale et al 1975, Rastin 1984, De Pascale et al 1993, and the two CAPRICE sets from Kremer et al 1999) excludes very recent results such as L3+C, WILLI and COSMO-ALEPH. All of the experiments included used curvature in a magnetic field for muon charge sign determination.

\subsubsection{CAPRICE}

The NMSU-WIZARD/CAPRICE magnet spectrometer measured ground level

muon flux and charge ratio at Lynn Lake (Manitoba, Canada, named the CAPRICE94 data set, taken in July 1994) and Fort Sumner (New Mexico, USA, named the CAPRICE97 data set, taken in spring 1997). It was sensitive to the range 200 $\mathrm{MeV} / \mathrm{c}$ to $120 \mathrm{GeV} / \mathrm{c}$, and their results are shown in Figure 3.3 [19].

The result for the low energy range between 0.2 and $0.55 \mathrm{GeV}$ is given for the CAPRICE 94 set as $1.21 \pm 0.03$ and $1.12 \pm 0.02$ for the CAPRICE 97 data set.

\subsubsection{WILLI}

The WILLI device at IFIN-HH at Bucharest, Romania has measured the charge ratio at low momentum (below $1 \mathrm{GeV} / \mathrm{c}$ ) [5]. It uses the observation of the reduced lifetime of negative muons that have stopped in matter for charge identification. Muons stopped in matter behave differently depending on their charge. Positives decay with their usual lifetime, but negative muons are captured in atomic 


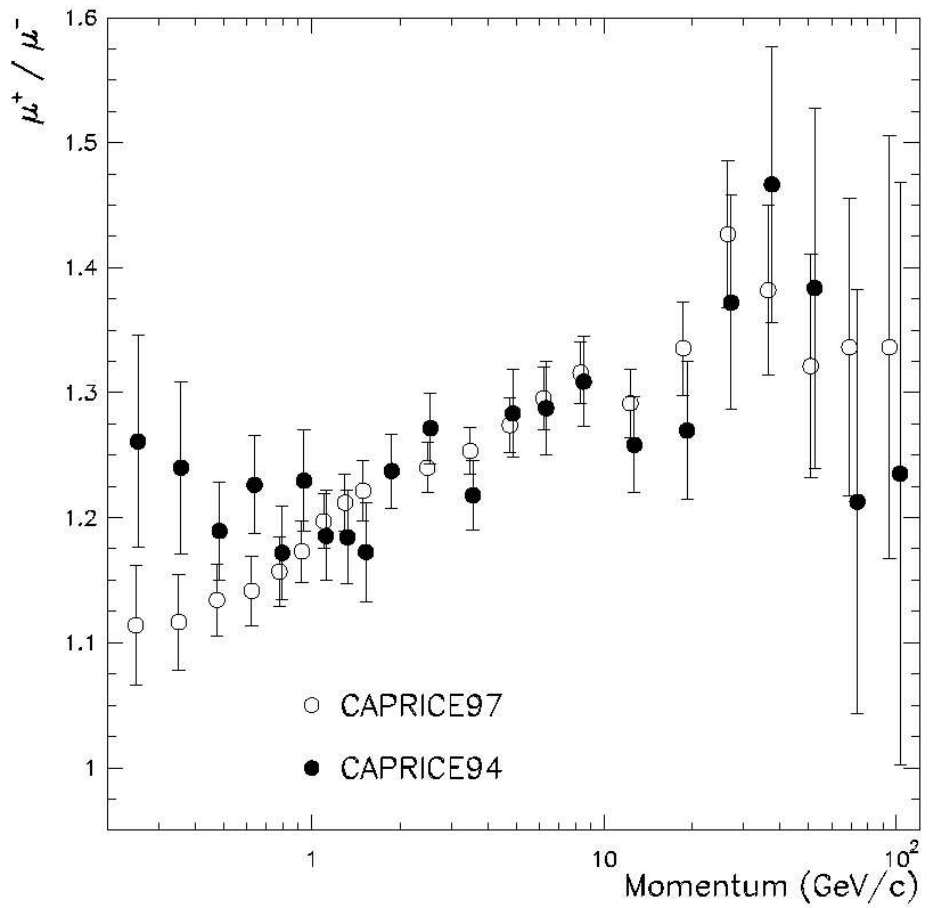

Figure 3.3: Charge Ratio versus momentum for CAPRICE. 
orbits, leading to a shorter lifetime of negative muons in matter, signalled by the timing of the appearance of decay electrons and positrons after the observation of a stopped muon. This method avoids the particle tracking problems with magnetic spectrometers. The detector observed a range from about .2 GeV to $1 \mathrm{GeV}$, with a charge ratio of 1.30 at $0.87 \mathrm{GeV} / \mathrm{c}$ and a ratio of 1.15 at $0.24 \mathrm{GeV} / \mathrm{c}$. Figure 3.4 confirms that the general increase in charge ratio at low energy is observed by both muon decay and magnetic spectrometers.

This comparison can also test systematic effects between two methods. The geomagnetic and other effects for the Fort Sumner (CAPRICE 97 data set) and Bucharest locations should be similar enough to cause the charge ratio measurements to be identical within errors. As will be discussed in Chapter 6, a measurement of a ratio such as this can be sensitive to both bias and background. A bias could take the form of charge dependent acceptance or unknown misalignment of the detector or magnetic field. Background is impurity in the signal typically caused by randomly assigning charge identification to muons that can't be measured. The WILLI measurements gave 10-20\% higher charge ratio than the CAPRICE 97 measurements, indicating there may be some systematic difference between measuring charge ratios with a magnetic spectrometer and a muon decay spectrometer.

\section{$3.2 \quad$ Underground Experiments}

Charge identifying detectors with overburden can sample a much higher energy range, but these are new experiments and were not included in the world survey above. 


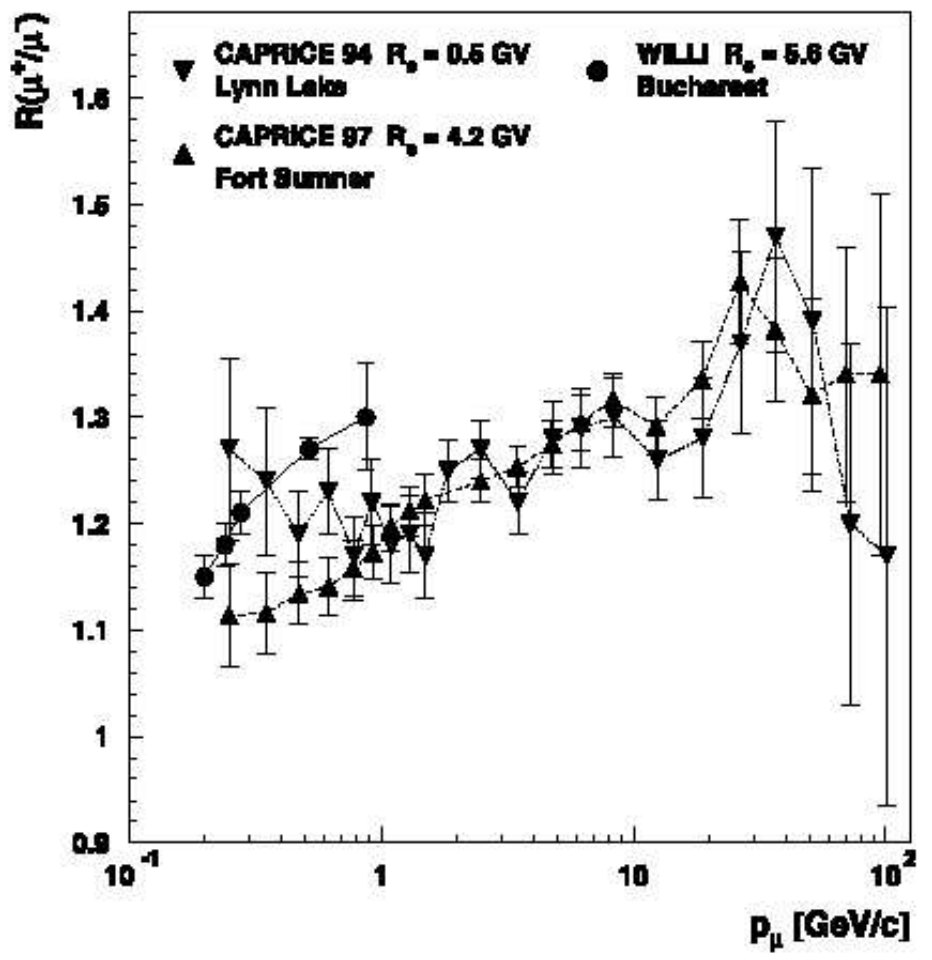

Figure 3.4: Charge Ratio versus momentum for the two experiments WILLI and CAPRICE, esp CAPRICE97 data, from [5]. 


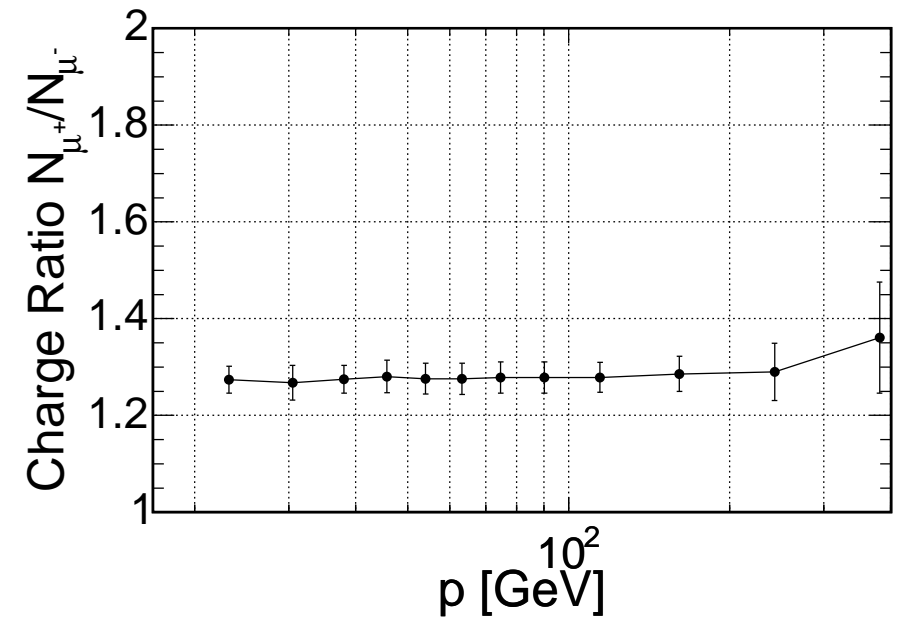

Figure 3.5: Charge Ratio versus momentum, data taken from tables in [6].

\subsubsection{L3 Experiment}

The L3 detector is located at the LEP collider at CERN, near Geneva, Switzerland. L3 used several timing scintillators and drift chambers to measure curvature in a $0.5 \mathrm{~T}$ magnetic field. It was upgraded with a TOF (time of flight) veto shield, called L3+C (L3+Cosmics), to aid in reconstruction of purely cosmic events. The detector lies under 30 meters of overburden, giving an effective surface momentum cutoff of $15 \mathrm{GeV}$. Results have been released by the L3 collaboration for muon charge ratio that covers the range just below the range of MINOS. The L3+C detector has a sensitivity to charge identification in the range of 20 to $500 \mathrm{GeV}$ [6]. This charge ratio is shown in Figure 3.5.

A value of $1.285 \pm 0.003$ (stat) \pm 0.019 (syst) was found, which is not inconsistent with the world average quoted above from [18]. It is interesting to note that L3+C used only $0.1 \%$ of their total data sample after all track quality cuts. 


\begin{tabular}{|l|c|c|c|c|}
\hline Experiment & $\frac{\mu^{+}}{\mu_{-}}$Ratio & Field & Size $m^{3}$ & Depth MWE \\
\hline \hline L3+C & 1.285 & $0.5 \mathrm{~T}$ & 1,000 & 80 \\
Cosmo-ALEPH & 1.278 & $1.5 \mathrm{~T}$ & 140 & 350 \\
MINOS & 1.347 & $1.5 \mathrm{~T}$ & 1,500 & 2000 \\
Caprice & 1.12 & $4 \mathrm{~T}$ & 0.5 & 0 \\
WILLI & 1.3 & Decay & 2 & 2 \\
\hline
\end{tabular}

Table 3.1: A Compilation of Charge Ratio Experiments and their Results and Specifics.

\subsubsection{Cosmo-ALEPH Experiment}

Cosmo-ALEPH uses the ALEPH collider detector at CERN, which is a magnetized hadron calorimeter and TPC scintillator tracker with an overburden of 130 meters, giving a surface momentum cutoff of $70 \mathrm{GeV}$. It has a maximal detectable momentum of $3 \mathrm{TeV}$. CosmoALEPH measured a charge ratio of $1.278 \pm 0.011$ (stat) in the range $80-2500 \mathrm{GeV}[20]$.

The $\mathrm{L} 3+\mathrm{C}$ and COSMO-ALEPH results give a good agreement of $1 \%$ over the energy range of $100 \mathrm{GeV}$ to $500 \mathrm{GeV}$. 


\section{Chapter 4}

\section{The MINOS Far Detector}

The MINOS Far Detector is located in the Soudan Underground Mine near Soudan, MN (47.82 ${ }^{\circ}$ latitude, $-92.24^{\circ}$ longitude). The Laboratory resides on the 27 th level of the mine, 2341 feet below the surface. A cartoon of the detector is shown in Figure 4.1. The detector looks like a 30 meter long cylindric octagon, and consists of alternating octagonal slices of $2.54 \mathrm{~cm}$ thick steel and one $\mathrm{cm}$ thick polystyrene plastic doped with scintillator. The planes are grouped into two "Supermodules" (SM), each a bit under 15 meters long.

One "plane" of the detector refers to two laminations of steel plus a single plane of scintillator, 8 meters in octagonal diameter. The total thickness of 2.54 $\mathrm{cm}$ steel, $1 \mathrm{~cm}$ scintillator, $1 \mathrm{~mm}$ of aluminum skin, and an air gap give an average spacing of $5.94 \mathrm{~cm}$. All 485 planes, except for the first steel only plane in each of two SM, are connected by optical fibers to photomultiplier tubes (PMTs) with readout electronics to measure the light output in the scintillator. A coil is wound through

the $30 \mathrm{~cm}$ diameter hole in the central, cylindric axis of each SM to generate a toroidal magnetic field in the steel. The detector's longitudinal axis, or z-axis, originates at the center of the first steel plane and points 26.554 degrees West of true North. The x-axis is in the local horizontal direction perpendicular to the $\mathrm{z}$ axis pointing generally to the West, while the $y$-axis is in the local vertical direction, 


\section{MINOS (Main Injector Neutrino Oscillation Search)}

\section{Far Detector}

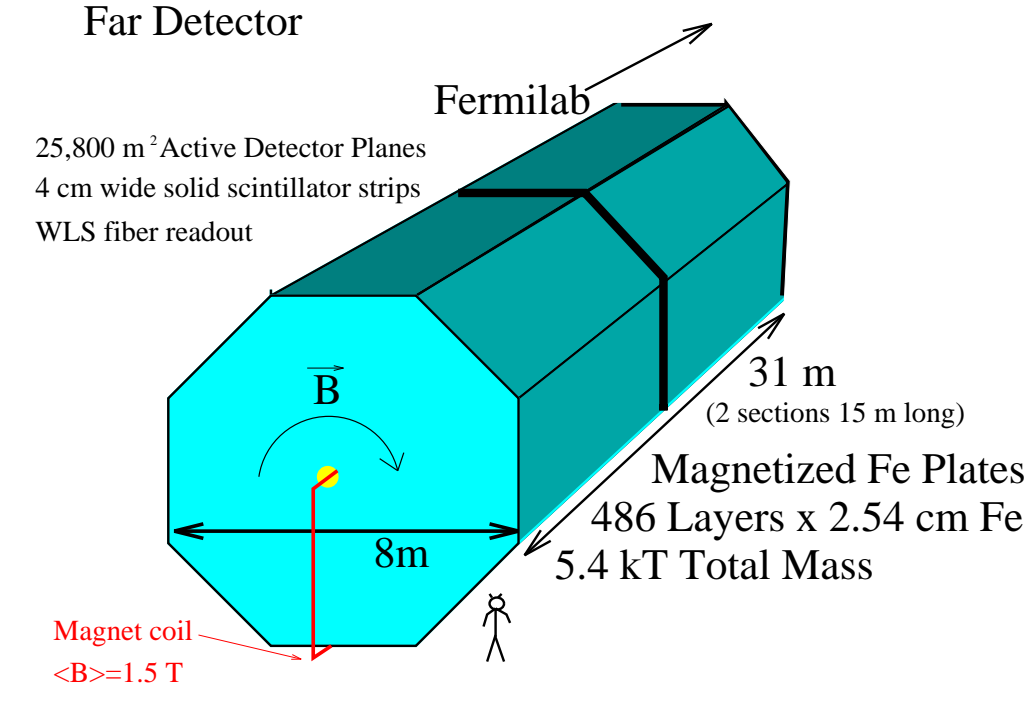

Figure 4.1: The MINOS Far Detector in graphical view. 
making a right handed coordinate system. The local detector origin is centered in $\mathrm{z}$ at the south face of the first steel plane and in $\mathrm{xy}$ at the center of that octagonal plane, in the middle of the coil hole.

\subsection{Construction of the Detector}

The Far Detector was designed around a constraint that all pieces of the detector had to fit in a $2 \mathrm{~m} \mathrm{X} 1 \mathrm{~m}$ X $8 \mathrm{~m}$ shaft and then be assembled underground. Construction of the detector proceeded by assembling steel laminations into an 8 meter octagon on a frame on the floor (this frame is called the "strongback"), welding them together, then placing scintillator modules atop this into a nearly 8 meter octagon and welding these in place. The 9 ton strongback, with the further 11 tons of steel and scintillator, is then lifted to the vertical by a 25 ton capacity crane on rails. The assembled plane is carefully walked down the detector hall into place on a set of detector rails, as shown in the picture near the top left and right sides of a steel sheet (the crane rails are near the top of the picture, above the entire detector on the right and left sides). These ears of the steel sheet are rested on the detector rails to hold the plane up. Each new plane is then bolted to the previous plane, and a $30 \mathrm{~cm}$ central collar is placed into the $33 \mathrm{~cm}$ hole in the center, bolted to the previous plane's collar to set the z positional alignment at the center. Each plane is also surveyed with an optical tracking device for alignment constants and finally connected to the optical readout system.

\subsubsection{Steel}

The steel planes are constructed from eight slices of steel, with four of those slices on each of the two laminations. Two trapezoidal and two rectangular slices are laid on a strongback and pushed together to make an 8 meter octagon. A second 


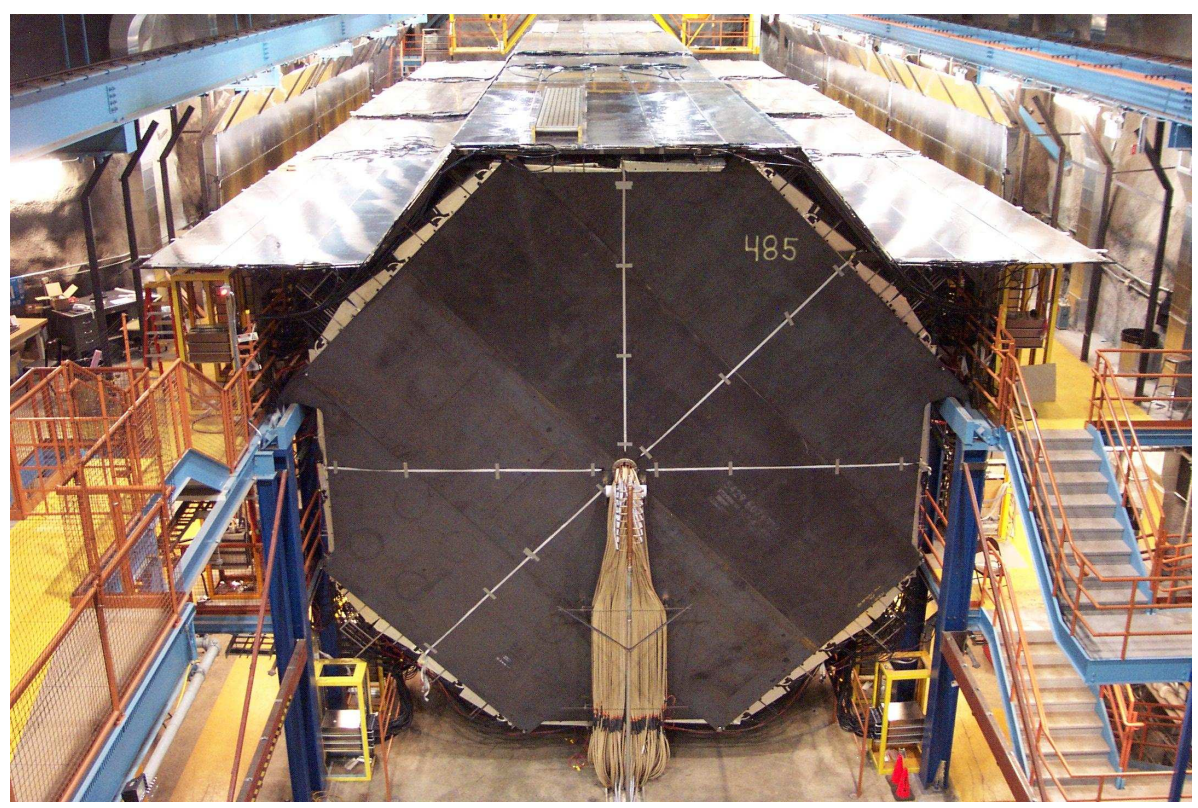

Figure 4.2: The MINOS Far Detector in the cavern hall.

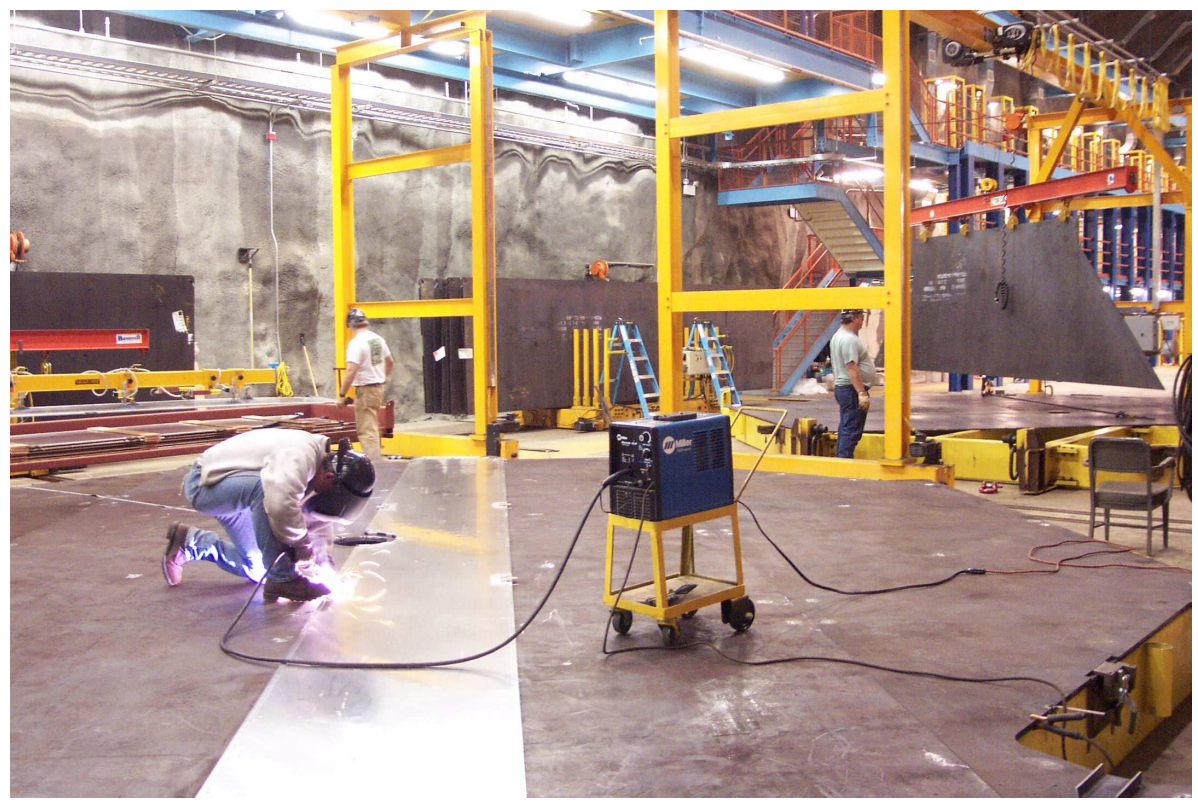

Figure 4.3: Construction of the MINOS Far Detector, two adjacent planes. The Southern plane is in the steel lamination phase, the Northern station has finished its steel lamination and welding phase and has begun to have its first scintillator module welded in place. 
layer of four more similar slices are laid upon this first later, but rotated by $90^{\circ}$ and pushed together and lined up to make a 1 inch thick, 8 meter octagon. The top lamination is plug welded in 72 1-inch holes to the lower lamination as in Figure 4.4 to create a steel plane which can be held up by its "ears". The ears are an extra bit of steel that was left on one side of each of the trapezoidal slices when they were cut. When finished, the plane will hang by the ears, but it will be bolted to previous planes by a steel bolt at each of the eight "axial" corners and will be offset from the previous plane at the central hole by a coil collar.

\subsubsection{Magnetic Field}

A toroidal magnetic field is generated by a $15 \mathrm{kA}$ coil through the central hole in the planes. The generated field goes from $1.7 \mathrm{~T}$ near the center to $1.2 \mathrm{~T}$ at 3.5 meters from the center. Since the field is contained within the steel, there is no simple method for measuring the field at points in the steel plane. Carving a hole in the steel to insert a hall probe simply causes the field lines to move around the hole. Initially, the field must be calculated, and a field map was generated with the ANSYS Finite-Element Analysis program, which is shown in Figure 4.5. This generator uses the basic geometry of the detector, including the coil return, the 8 slices in two laminations with an average gap between slices and the average chemistry of the steel to return an average field map for the entire detector. Different field maps for the air field between steel and for the end planes have also been generated. The field in air is generally a factor of 500 smaller than in the steel, and the end planes

of each SM are affected mostly by proximity with the vertical portion of the return coil at the four SM ends.

Each plane will be slightly different from the model due to gaps between the eight slices being different and from the complication of the chemistries of the 


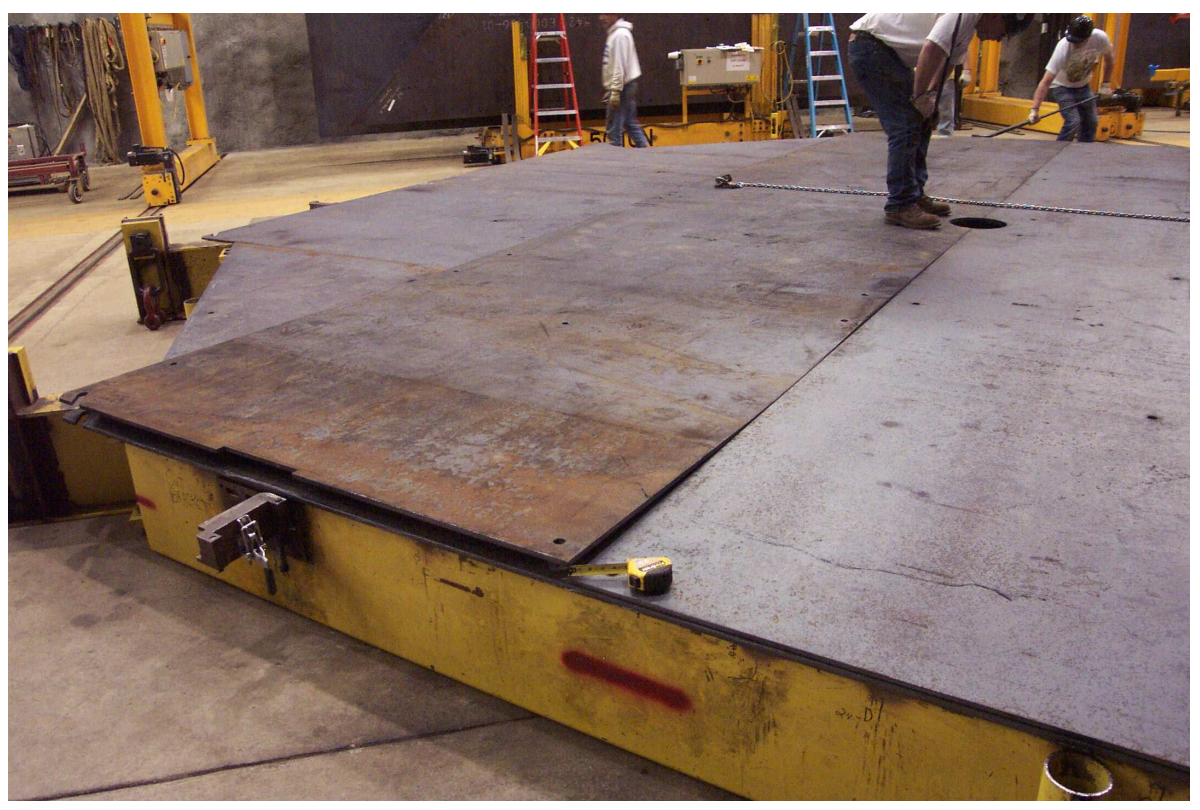

Figure 4.4: Steel Laminations of a steel plane. 
steel. During the construction phase, steel slices were delivered from 59 different "heats". One heat refers to a large crucible containing large quantities of iron and other material melted together and poured into forms and sold to many different customers. The heats all vary slightly in chemical composition, which can affect the magnetic properties of the steel. In particular, the B-H hysteresis curve gives the saturation of the steel, which for the chemistries used in MINOS can vary by several percent. A further complication is that steel from up to eight different heats were used in a single plane, with the average number of heats per plane being between three and four. To account for all the variations within the construction of a plane, an induction coil was wound around every steel plane, to be read out by the BDOT system during coil ramp up and cycling. The chemistry of each heat is measured by cutting a small ring of steel out of each plane and measuring induction to get a B-H curve. A toroid is wrapped around the ring and ramped up in current, while a second sense coil is wrapped around the same ring and reads out the induced current. This data is intended to be used for overall normalization of the magnetic field in each plane, but requires further study.

\subsubsection{Scintillator}

The scintillator is made of adjacent $4.1 \mathrm{~cm}$ wide, $1 \mathrm{~cm}$ thick strips that are up to eight meters long of plastic polystyrene doped with PPO and POPOP organic fluors. Each strip is co-extruded with a thin layer of Titanium Dioxide, to internally reflect any light. The extrusion process included a thin groove along the front of one wide side of the strip. This groove is the only part of a strip that is not encased in $\mathrm{TiO}_{2}$ because it contains a wavelength shifting fiber (WLS) to collect and reemit the UV light from scintillation as green light. The bialkali photocathode of the PMT's has a decreasing quantum efficiency as wavelength increases above $550 \mathrm{~nm}$ 


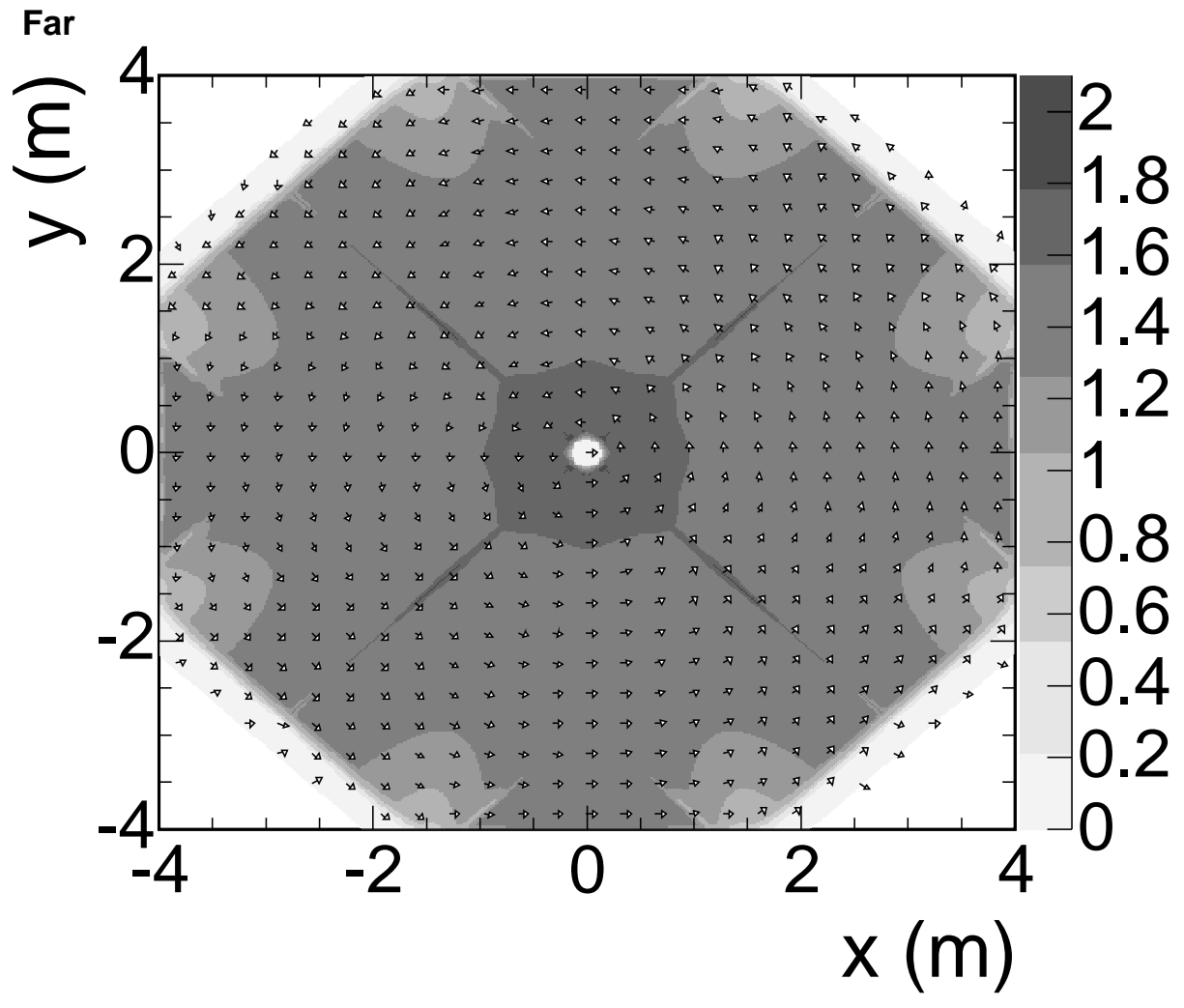

Figure 4.5: Magnetic Field in a steel plane. 
and essentially zero efficiency for ultraviolet light. The WLS fiber has an attenuation length of 5 meters which is acceptable considering the maximum distance travelled by photons in this material is 8 meters and the average distance travelled is closer to 4 meters. At the ends of the scintillator modules, the light output is routed to the PMTs by clear fiber which has a greater attenuation length of 11 meters.

The $1.2 \mathrm{~mm}$ diameter WLS fiber is placed in the groove and runs the length of a scintillator strip, which is between 3.3 and 8 meters long, depending on the location of the strip within the plane. The fiber groove is light sealed with metallic tape, and the strips are glued to an aluminum base and the groups of 20 or 28 strips then have an aluminum top crimped on. The fibers at both ends of the module are collected in a light tight housing, terminated with a polished flat connection junction, to be mated with a clear fiber connection and some optical grease. The clear fiber connected to the strip is read out by connection with a PMT.

One scintillator plane is made up of 192 such strips, each spanning the distance across a plane. Each scintillator plane is either a U-view or V-view plane, which refers to the axis that plane measures charged particle tracks with respect to. These axes are nominally $45^{\circ}$ to the $\mathrm{XY}$ axes, in particular the U-axis is between $\mathrm{X}$ and $\mathrm{Y}$ axes and the $\mathrm{V}$-axis is between the $\mathrm{Y}$ and the $-\mathrm{X}$ axes.

To mechanically contain the strips, they are grouped in either 20 or 28 strips into a scintillator "module". There are 6 different types of module in a plane: $\mathrm{A}, \mathrm{B}, \mathrm{C}, \mathrm{D}, \mathrm{E}$, and $\mathrm{F}$, constructed in the pattern $\mathrm{A}, \mathrm{B}, \mathrm{C}$ or $\mathrm{E}, \mathrm{D}$ or $\mathrm{F}, \mathrm{D}$ or $\mathrm{F}, \mathrm{C}$ or $\mathrm{E}$, B, A. U-planes were built with $\mathrm{E}$ and F modules; V-planes were built with $\mathrm{C}$ and D modules. The structure is diagrammed in Figure 4.8. The A and B modules are the outer two on top and outer two on bottom and contain 28 strips each, and it can be seen that these must be trapezoidal and progressively narrower to more fully cover the octagon shape of the steel plane. These two module types were constructed 


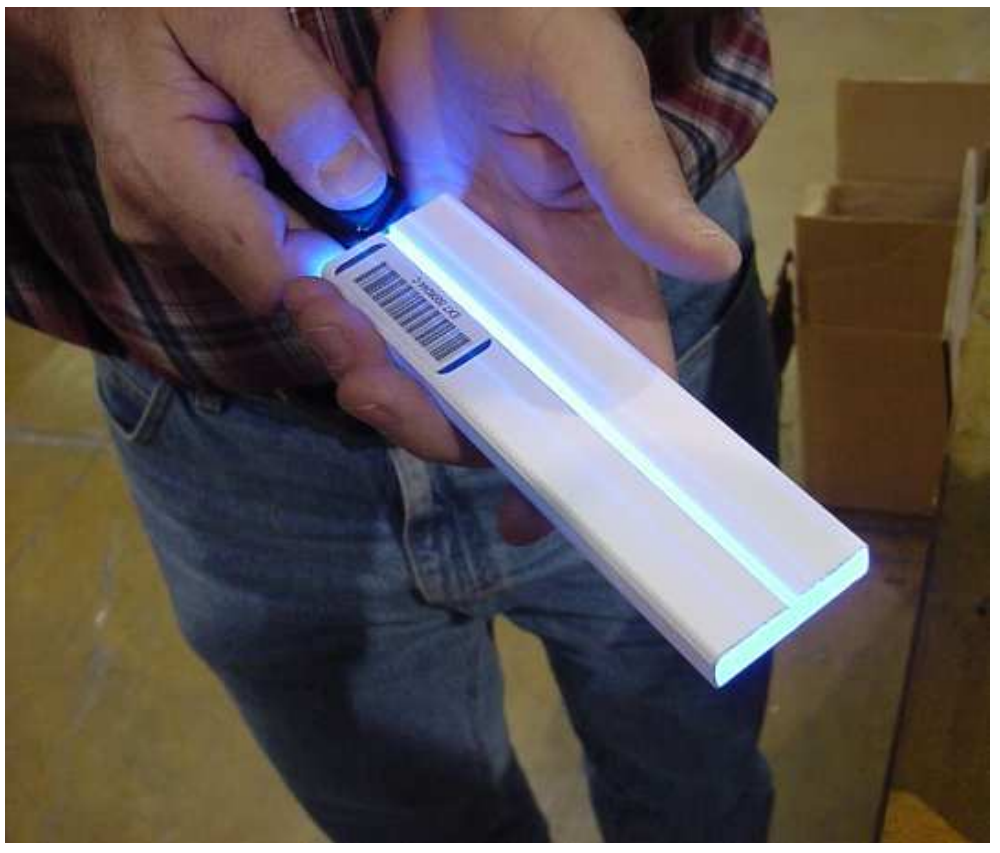

Figure 4.6: Scintillator strips with the WLS fiber. 

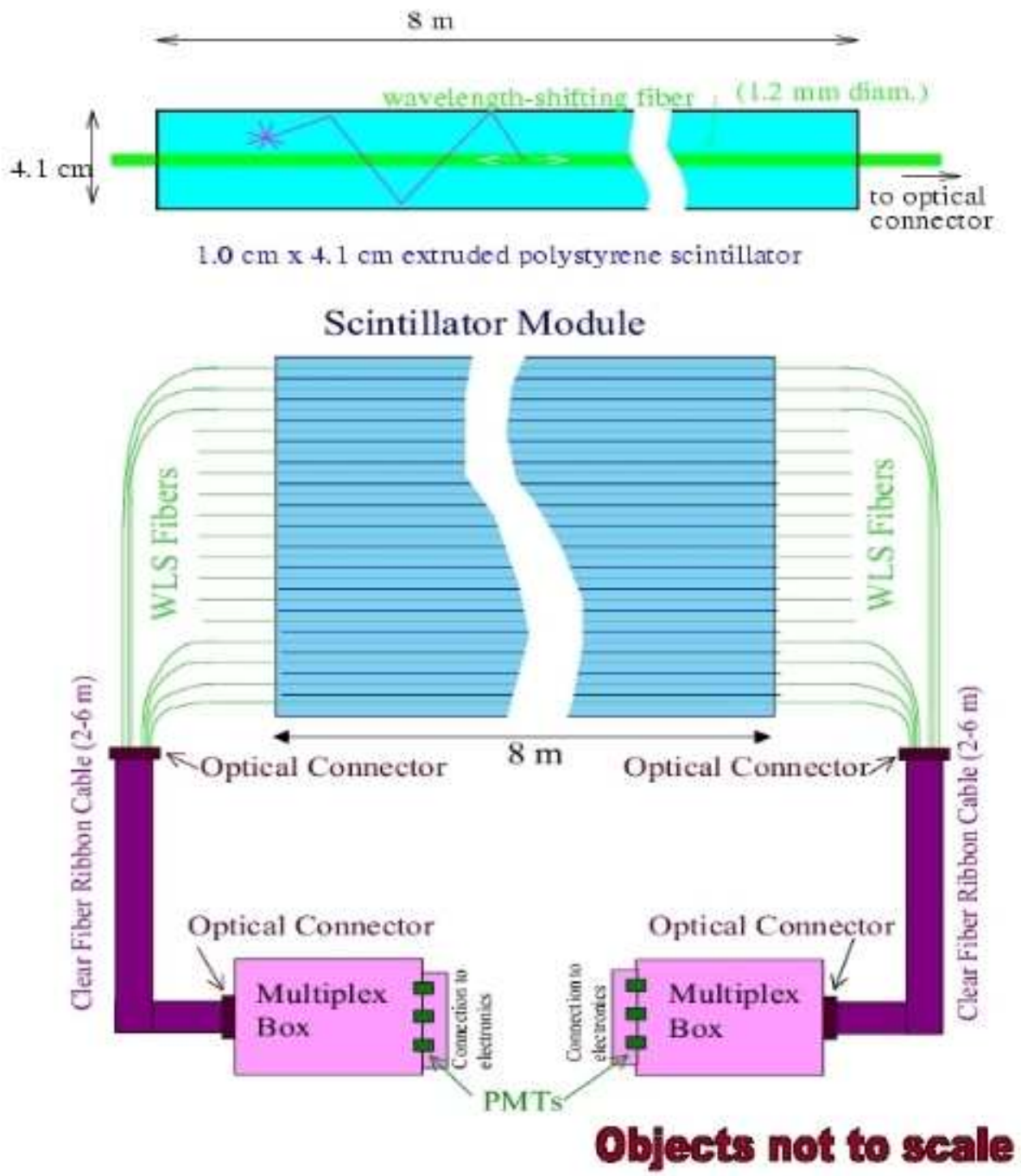

Figure 4.7: Scintillator strips in a module. 
at the University of Minnesota Module Factory and "mapped" at that factory for light output and other strip characteristics. C,D,E, and F contain 20 strips each and are the central four modules. These modules were constructed at the California Institute of Technology Module Factory and were also mapped at that factory. The inner two contain a hole between them, for the coil to run through, and this hole required a bypass for the WLS fiber for the strips affected. Some number of the modules were also mapped at the mine, to ensure the module was not affected by the journey. The mapping process consists of illuminating the scintillator in the module with a radioactive source moved above the module with precisely controlled stepper motors. The light output is used to calibrate for light output level, attenuation and position of the strips.

The scintillator sheets consist of 8 modules arranged on a steel plane after the steel plane is welded together as shown in Figure 4.9, where the skin of the module can be seen. As each scintillator module is placed, it is welded at the corners with steel clips to hold them in place. Clear fiber connectors are attached to the WLS terminations at this point and bagged in preparation for the plane raising. When finished, a scintillator sheet contains 192 strips in the eight module groups of 20 and 28 , and the module edge strips are separated from adjacent module edge strips by 5-7 millimeters. The direction of the scintillator strips changes by 90 degrees about the transverse axis for every other plane to give spatial resolution. Scintillator is considered to be in a $\mathrm{u}, \mathrm{v}$ axis rather than an $\mathrm{x}, \mathrm{y}$ axis, which is merely rotated -45 degrees from the $\mathrm{x}-\mathrm{y}$ axis. If scintillator strips are perpendicular to the $\mathrm{u}$-axis, the entire plane is called a U-plane, and for a scintillator plane perpendicular to the v-axis, it is called a V-plane. Even planes in SM1 are U-planes and odd planes are V-planes. In SM2, it is reversed to even planes being V's and odds being U's due to the one steel only plane at the face of SM2. 


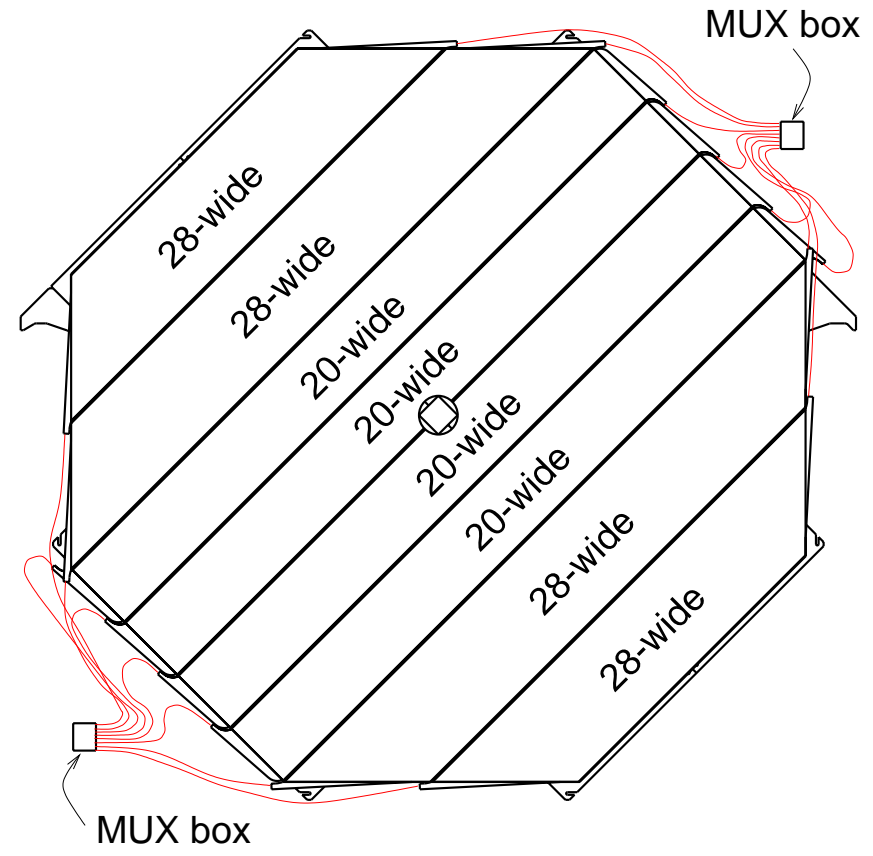

Figure 4.8: Modules in a plane.

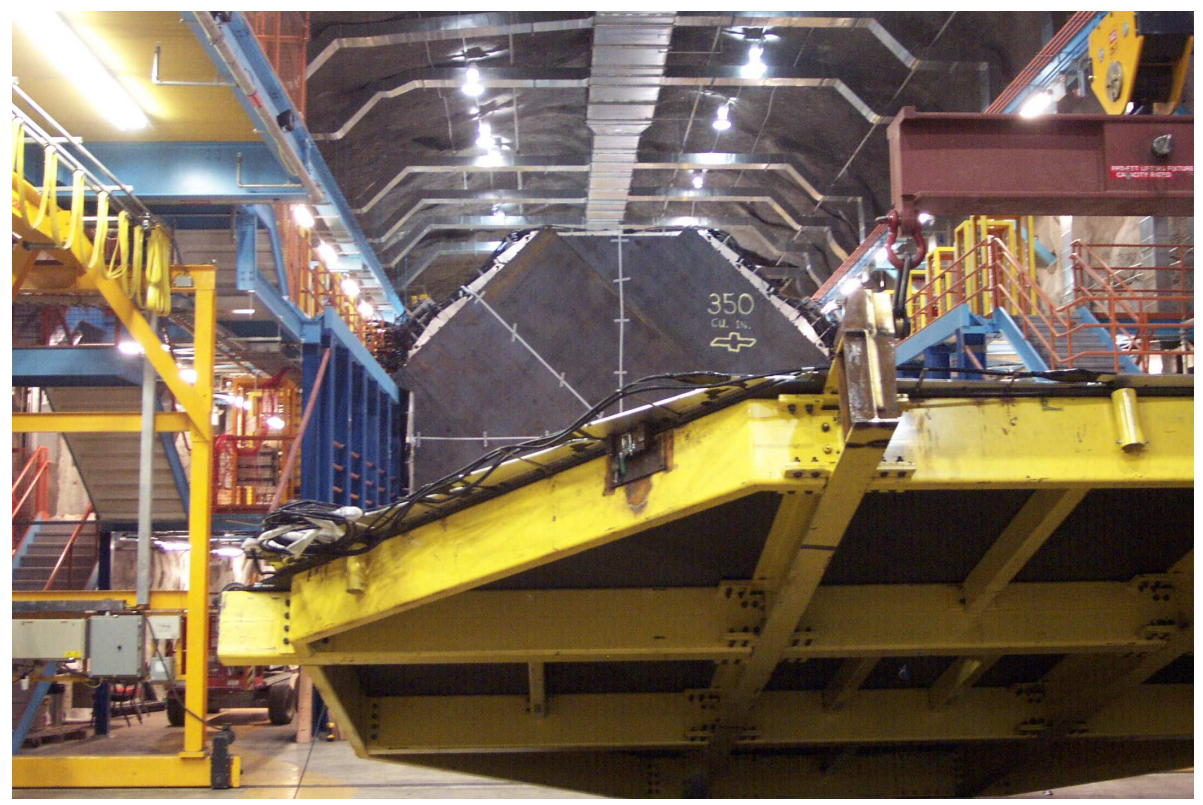

Figure 4.9: Lifting a finished plane. 
The clear fibers carrying the light from a scintillator module were mated onto a Hamamatsu R5900U-00-M16 (M16) phototube, a multi-anode PMT with 16 pixels, each of size $4 \mathrm{~mm} \mathrm{X} 4 \mathrm{~mm}$ in area. To reduce costs, the light output from 8 particular fibers was summed at one pixel so that for 192 fibers at each end of a plane, only 3 PMTs (1.5 at each end) are needed to read out the entire plane. At the two ends of a plane, the fibers were routed into a multiplexing box (MUX box) containing 3 PMTs. This MUX box can service two planes, and due to the alternating order of U-View versus V-View planes, each MUX box was connected to planes in the same view separated by one plane.

The multiplexing scheme treats the two ends slightly differently. For one end, non-adjacent strips separated in space by 15 strips were ganged together to one pixel. So all 16 pixels of one PMT plus 8 of another PMT are needed. At the other end, a different ordering was used to gang together fibers [21].

\subsubsection{Completion of Supermodules}

When each plane was finished, it was clipped to the strongback frame and the frame was then lifted from the north end, or top end. A completed plane weighs approximately 11 tons, the frame is a further 9 tons, so care was taken in moving it down the hall with the 25 ton capacity crane.

After 248 planes were lifted and hung in this manner, the coil was installed. First, a copper jacketed tube containing copper water cooling pipes was inserted in the coil hole. The coil hole is defined by the coil collars that are installed at the center of each plane in the central .15 meter radius hole during construction. It has a .17 meter outer radius and .13 meter inner radius as in Figure 4.12, while the copper cooling tube (.125 meter radius) must fit inside this hole made by jointing these 249

collars for SM1 (planes 0 through 248) or 237 collars for SM2 (planes 249 through 


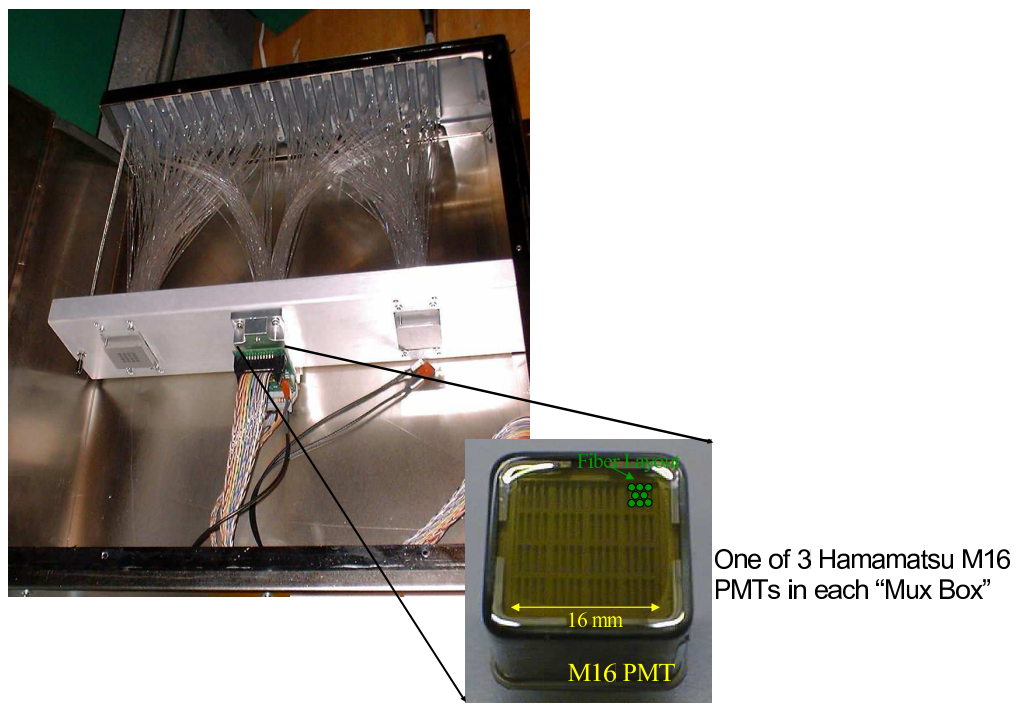

Figure 4.10: A MUX box which typically contains three PMTs.

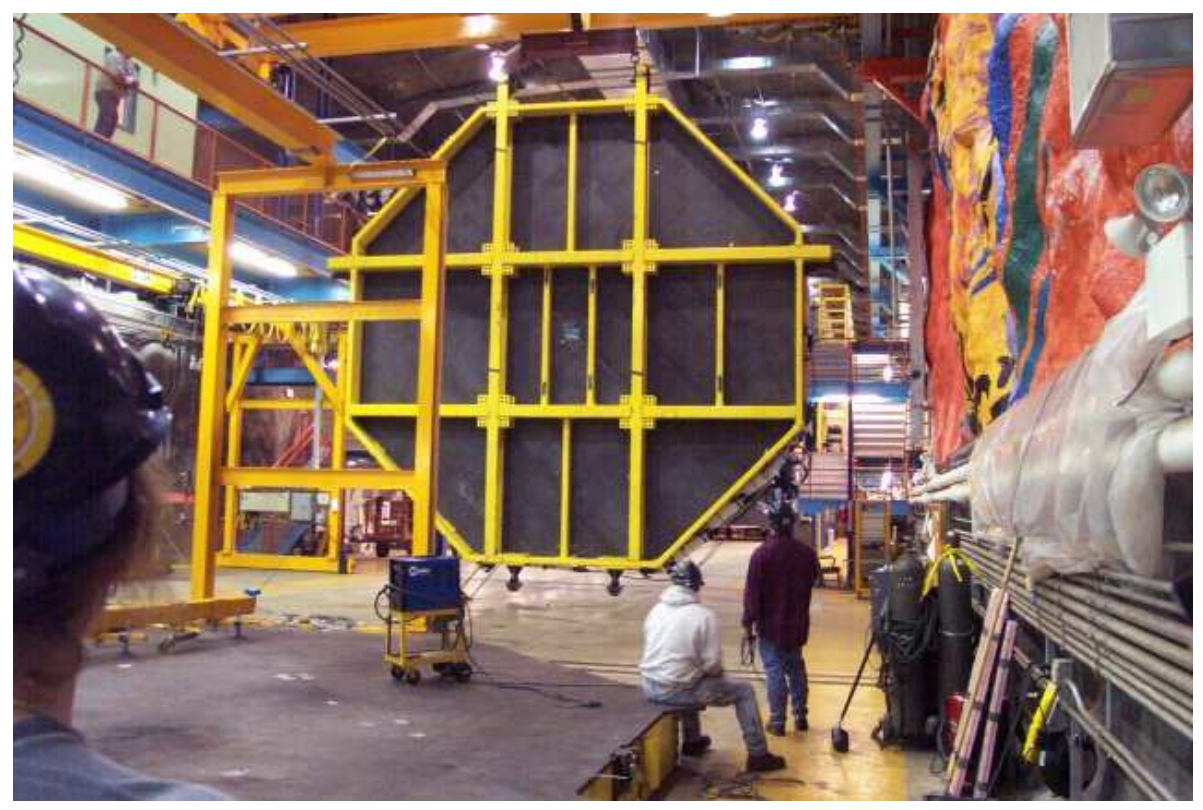

Figure 4.11: A strongback carrying a completed plane. 
485) together. Once this was in place, 1 inch diameter copper conductor wires were drawn down this tube until 190 wires were in place. These wires were spliced to make a 190 turn coil that went through the central axis, down from the center at one face of the SuperModule (SM), underneath the detector through a trench (also water cooled) kept at a distance of 1.5 meters, and up to the center at the opposing face of the SM to meet with the next turn. The first and last wires were linked into a constant current 80 Ampere power supply to provide a $15.2 \mathrm{kA}$-Turn dipole loop. The second SM was constructed in the same manner, including its coil and its first steel only plane. The first SM has 248 scintillator planes while the second SM has 236 scintillator planes.

\subsection{Detector Readout and DAQ}

The electronics readout of the Far Detector was designed based on the low rate of events expected. The signal is dominated by dark noise from the PMTs, which is less than $1 \mathrm{kHz}$ per PMT on average, so a single high-speed digitizer can read out many PMTs and still achieve low dead time. The first step of the electronics readout is the VA chip series from IDE Corp. (Oslo, Norway). The chip is made up of 32 channels of charge sensitive preamp, shaper, and a sample/hold circuit. Finally, the output signals go through an analog output multiplexer. One VA reads out a single PMT and is triggered based on the common dynode signal of the PMT [22]. Since the Hamamatsu M16 PMT consists of 16 anode channels, half the VA chip's channels were not needed. Seventeen channels were used; one extra channel was connected to a PIN (photo-injection) diode for monitoring of the light injection (LI) calibration system. Three such chips per VA Front-end Board (VFB) monitor the MUX box connected to one end of either two even or two odd planes.

The front end crates are installed on the lower and upper levels on each side 


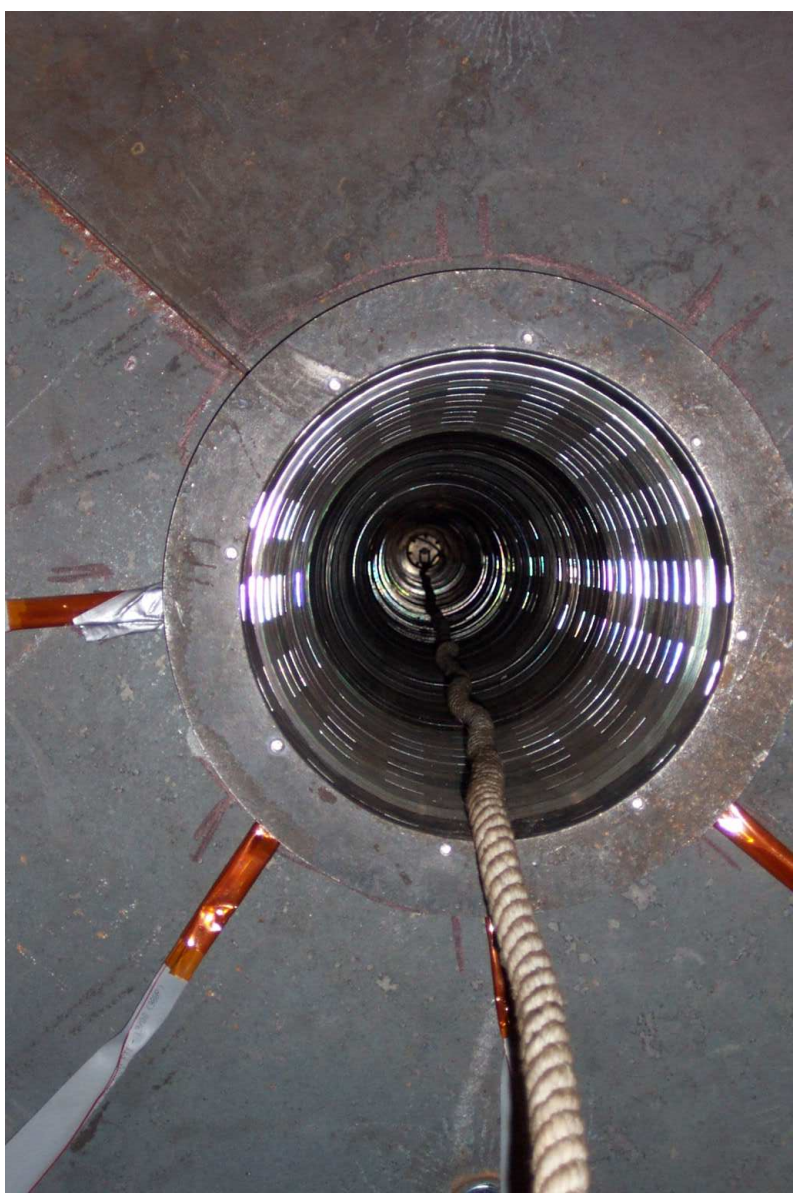

Figure 4.12: The Coil Collars making up the Coil Hole. 


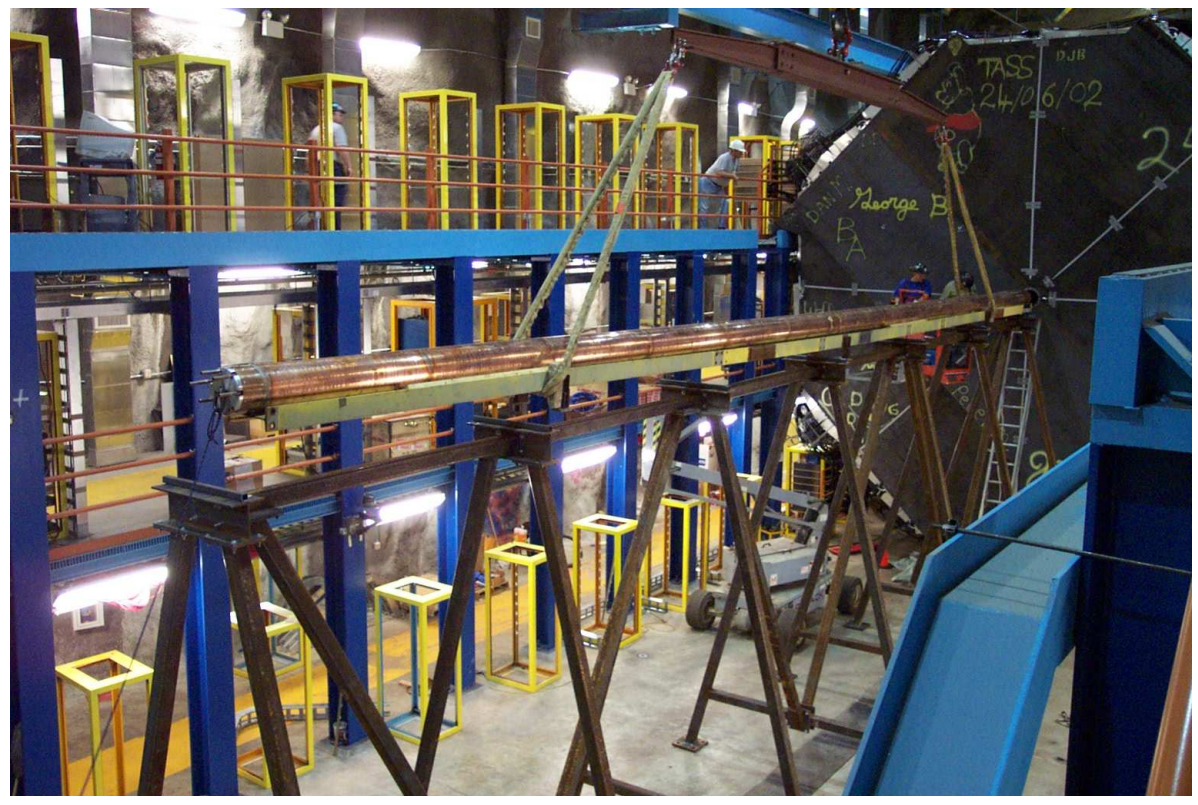

Figure 4.13: Insertion of the magnetic coil tube through a Supermodule.

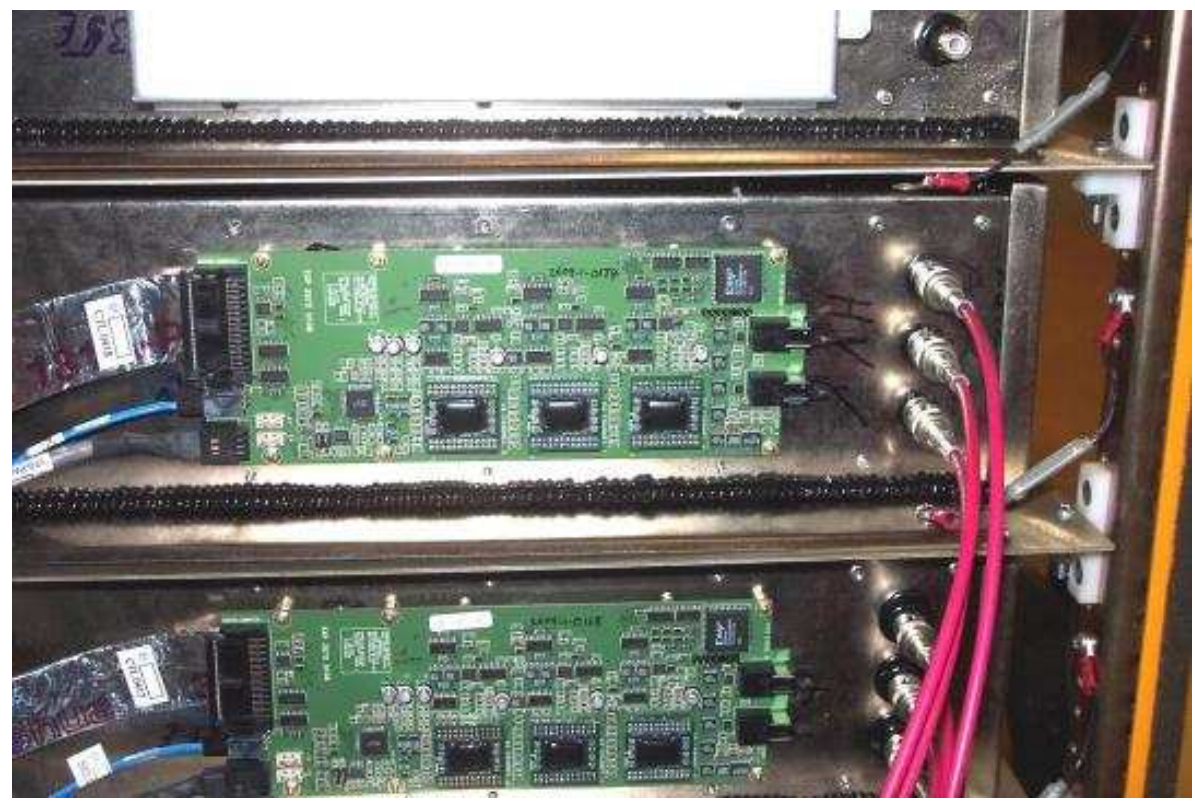

Figure 4.14: A Far Detector Front End Electronics (FEE) board. 
of the MINOS hall to be close to the optical output of the scintillator planes. The front end crates are read out by the DAQ (Data AcQuisition) crates in the middle level. Each front end crate contains eight MUX boxes and the eight associated VFBs. Each VFB is controlled and readout by a VARC (VA Readout Card), so the control cables, power supply, and analog output cables must be routed to the second level, to a DAQ crate, which hold up to three VARCs. Each VARC can monitor 12 VFBs, or 36 VA chips or 36 PMTs, by use of one 14-bit ADC (Analog to Digital Converter) for every 2 VFBs. Since 1.5 PMTs readout one side of a plane, a single VARC is connected to one side of at most 24 contiguous planes. The readout chain is shown in Figure 4.15 from VA chip to DAQ data stream at the final collection point. 3 PMTs on both sides readout two non-adjacent planes, 3 PMTs per FEE board with one VA chip for each PMT, 2 FEE boards readout per ADC, 6 ADCs per VARC, 3 VARCs per crate, and 16 crates for the whole detector. In Figure 4.15, the PMTs are at the top, connected to VFBs. The VFBs feed into VARCs and the data which passes a VARC-level trigger discussed in the next section is fed into the trigger processor (TP, which makes higher level triggering decisions) through the branch readout processor $(\mathrm{BRP})$. This process is shown with a more vertical view of just one channel in Figure 4.16, with the output of a scintillator going through a PMT to a VFB to a VARC, to the BRP and TP to the final data collection point DAQ.

Each DAQ crate contains a timing card (TRC in Figures 4.15-4.16) connected by measured lengths of fiber to the GPS (Global Positioning System) crate which provides nanosecond level timing (1.6 nanosecond from timing calibration) to the crates, the three VARCs, a ROP (ReadOut Processor) board, and is daisy-chained through an electrical PVIC (PCI to PCI Vertical transparent InterConnection) cable to up to 5 adjacent DAQ crates (grouped together as a "branch"). Each DAQ crate's 


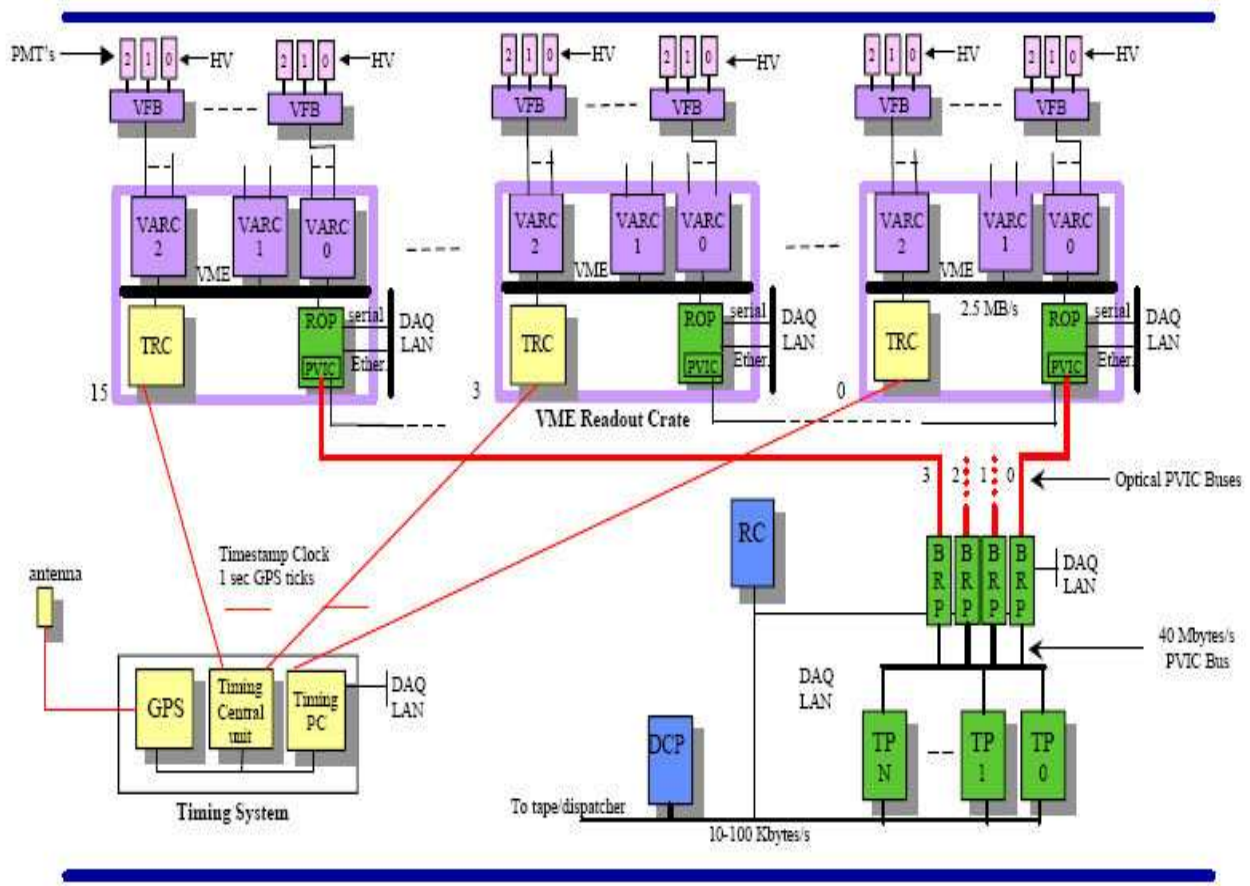

Figure 4.15: The Far Detector DAQ readout chain. 


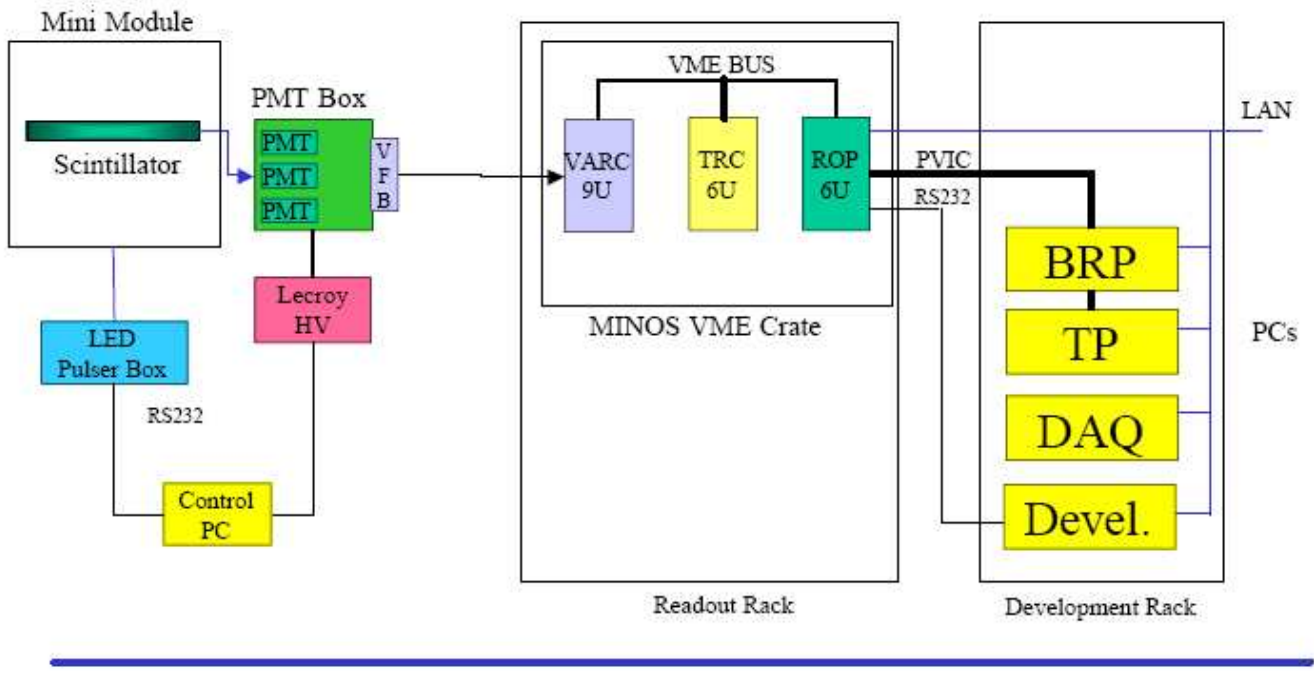

Figure 4.16: A more vertical view of the Far Detector DAQ readout chain. 
ROP assembles the timestamped data into single second-long timeframes and passes the data along the PVIC chain to the final DAQ crate. The output of the head node of a branch is sent by optical PVIC fiber to the branch's BRP. The BRPs communicate with the trigger farm (TP) to get all the data for the detector sorted in one place. The output of the TP is sent on to the DAQ data collection processor (DCP) depending on how the data passes any defined triggers. This output is stored in root files as binary data storing the logical electronics channel, timestamp and pulseheight collected on that channel.

\subsubsection{DAQ Triggers}

The DAQ triggers consist of a two hardware triggers, the dynode and the VARC triggers, and an upstream high-level software trigger called the 4/5 trigger. The dynode trigger is based on the common dynode signal of a single PMT. This signal is watched by the VFB, and once the preset level is reached, the VFB notifies the VARC that data is waiting for readout and also signals the three VA chips to begin a sample and hold on every channel in parallel. The time between initial signal at the PMT and the sample and hold command being issued is approximately 200 ns. The VARC then reads out each of the 16 channels on whichever of the $3 \mathrm{VA}$ chips have registered a dynode trigger one by one through the 14-bit ADC. This readout takes $5 \mu \mathrm{s}$ for one VA chip, and since as many as 6 VA chips on two VFBs may pass the dynode trigger, a deadtime of at most $30 \mu$ s exists between the sample and hold process beginning and the full readout. The VARC uses a $640 \mathrm{MHz}$ clock for time-stamping, and so there is a 0.45 ns time resolution on each dynode trigger and must therefore be the same timestamp for all channels on a particular VA chip.

The dynode trigger data is passed to the VARC Sparsifier, which does pedestal and common noise subtraction and most importantly, removes all channel readings 
below the threshold of $1 / 5$ th of a photoelectron [23]. Due to higher noise rates than expected, a further VARC level trigger was implemented. For data from a VARC to be readout, any two out of as many as 36 total VA chips on the same VARC must be triggered within 500 ns of each other.

After the hardware triggers are passed, the data is fed together to the TP, and the data is ordered by time and by crate. The data stream is divided up into chunks of hits separated by at least 100 clock cycles (156 ns). These chunks are termed candidate events and are examined for whether or not any four out of five contiguous planes in the detector contain hits. Candidates passing this final trigger are called "snarls" and passed along to the DCP to be stored as the final raw DAQ data to be processed in the offline by the reconstruction.

\subsection{Reconstruction}

At the Far Detector, reconstruction begins by associating electronics "digits" with physical scintillator strips correlated in time and space [24]. A CandDigit corresponds to the timing and pulseheight observed on one pixel of a PMT, and refers to any signals within $75 \mathrm{~ns}$ of the trigger being summed together, followed by $5-30 \mu \mathrm{s}$ of readout deadtime. Due to the optical multiplexing at the scintillator to PMT level, CandDigits can be associated with any one of eight strips, and demultiplexing must be performed before associating the digits with the correct strips. Demultiplexing for cosmic ray muons (each type of event requires different reconstruction) proceeds by associating CandDigits with planes, finding the likelihood that the digits are crosstalk and depending on whether two CandDigits can be associated with a single strip (if one channel out of the eightfold ambiguity on one end matches with a possibility from the other side, then the two CandDigits are certainly opposite ends

of the same strip), either simply associates them or passes their information into a 
sliding window algorithm if only one end was hit[21]. Once this is done, the one or two associated CandDigits are referred to as a CandStrip, as one physical scintillator strip in space. An example of demuxing can be seen in Figure 4.17.

\subsubsection{Calibration}

Before the CandStrips are used, the known response of each strip is applied to the CandStrips to end up with a linearized detector. These calibrations include the pedestal subtraction of the front end electronics, pulse height linearization, gain calibration, attenuation constants and timing. However, these are not as important for cosmic muons since their behavior is much simpler than other particles MINOS was designed to examine, such as hadronic and electron showers and so it is not discussed here, see [25].

\subsubsection{Track Finding}

The Hough transform is used to find tracks. It takes the event strip hit list after demultiplexing (all strips with charge, including noise and crosstalk hits) and returns a track strip hit list. It transforms MINOS events in U,Z or $\mathrm{V}, \mathrm{Z}$ space into Hough space. If these transformed points in U,Z space are contiguous and form a straight line, the lines in Hough space converge to a peak as in Figure 4.18 and it becomes straightforward to winnow hit strips in a track from noise hits. The performance and details of the inner workings are listed in "Appendix A: The Hough Transform" of David Petyt's thesis [26]. Real curvature in the track, whether from magnetic bending or from multiple scattering, broadens the peak in Hough space. From D. Petyt's conclusions, the efficiency of the algorithm for finding straight tracks and removing noise hits increases as the number of bins in the Hough space is increased, although efficiency for finding tracks containing curvature favors 


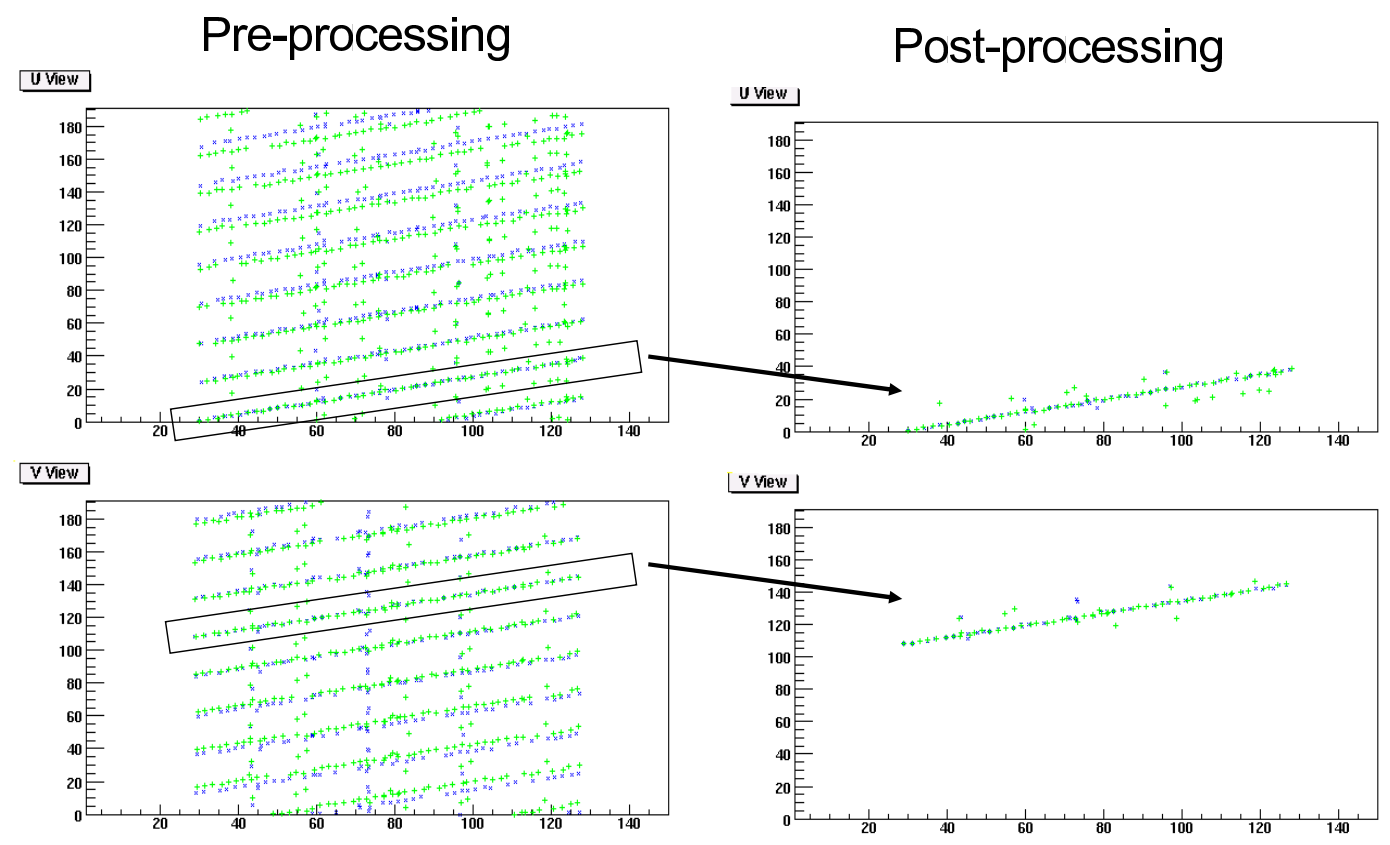

Figure 4.17: The demultiplexing concept: on the left is possible strip combinations versus $\mathrm{Z}$ ( $\mathrm{U}$ view on top, $\mathrm{V}$ view on bottom). Note that each possible track has two colors, light for East, dark for West and these don't necessarily match up. On the right, only the matched up East and West possibilities are plotted as the correct demultiplexed track. 
decreasing the number of bins. Efficiency versus event length reaches maximum very sharply but depends on the bin size used. For the cosmic muon reconstruction, a bin size of 40x40 was chosen, which reached maximal efficiency near when a track had only 7 or more planes as in Figure 4.19, so track finding is quite efficient even with few planes.

The end result is the list of transverse positions of the centers of hit strips that are associated with a track. Since the track went through an unknown part of a strip, the track must be fit to determine the likely trajectory.

\subsubsection{Track Fitting}

The track fitter takes the track strip hit list and produces a fit to these hit positions using a Kalman filter. The Kalman filter requires a model for the data, an error estimate, and the "noise" representing energy loss and multiple scattering in material. An infinite negative momentum is input to the iterative CDHS least squares algorithm, essentially a Kalman filter, which converges very quickly on a physical solution. It is described in detail in [27] and the performance of this algorithm is studied in [25].

The fractional error on the muon momentum measurement is limited by multiple coulomb scattering and it decreases to $10 \%$ as tracklength goes to 10 meters [28]. The output of the fitter is a $\mathrm{q} / \mathrm{p}$ measurement at each plane, as well as the direction cosines of the track at each plane. The initial momentum of the track is then $1 /(q / p)$ at the vertex plane and the fractional error on $q / p$ is given by the track

fitter. This fractional error is used to determine the confidence of $q / p$ identification (QPID). 
Track in X,Z space

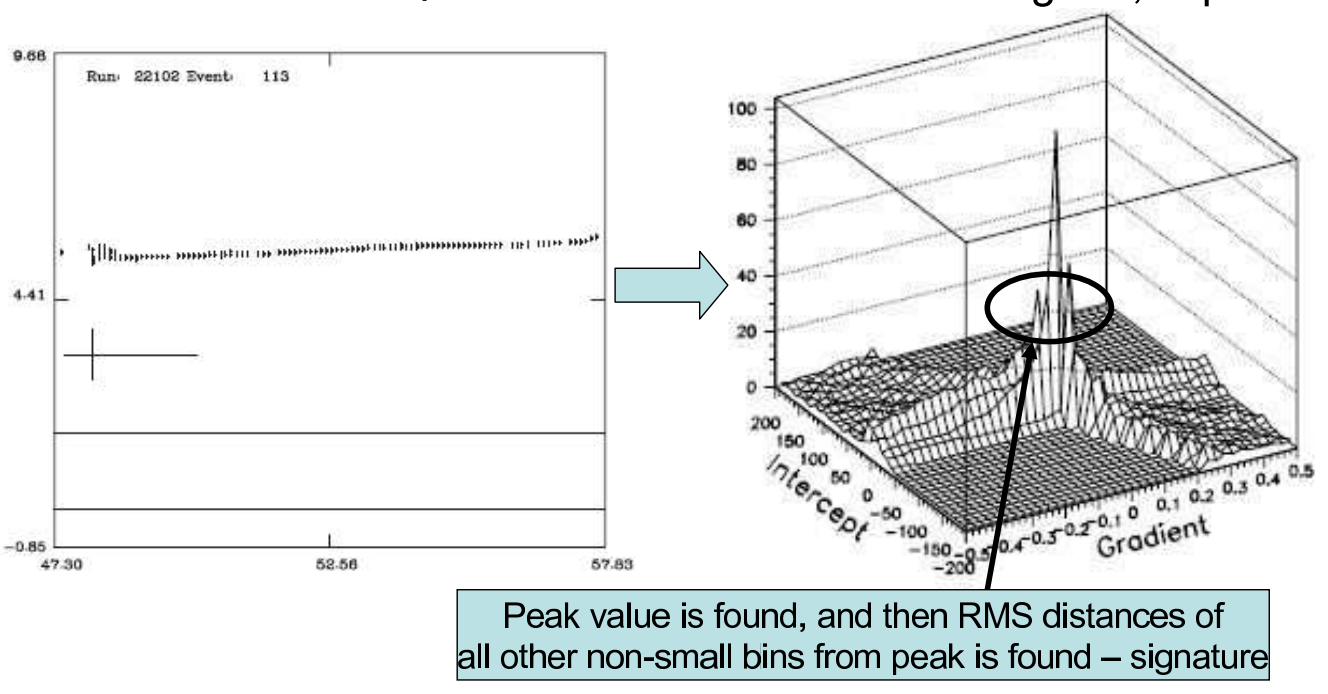

Figure 4.18: A track in x,y space translated into Hough space (D. Petyt thesis). 


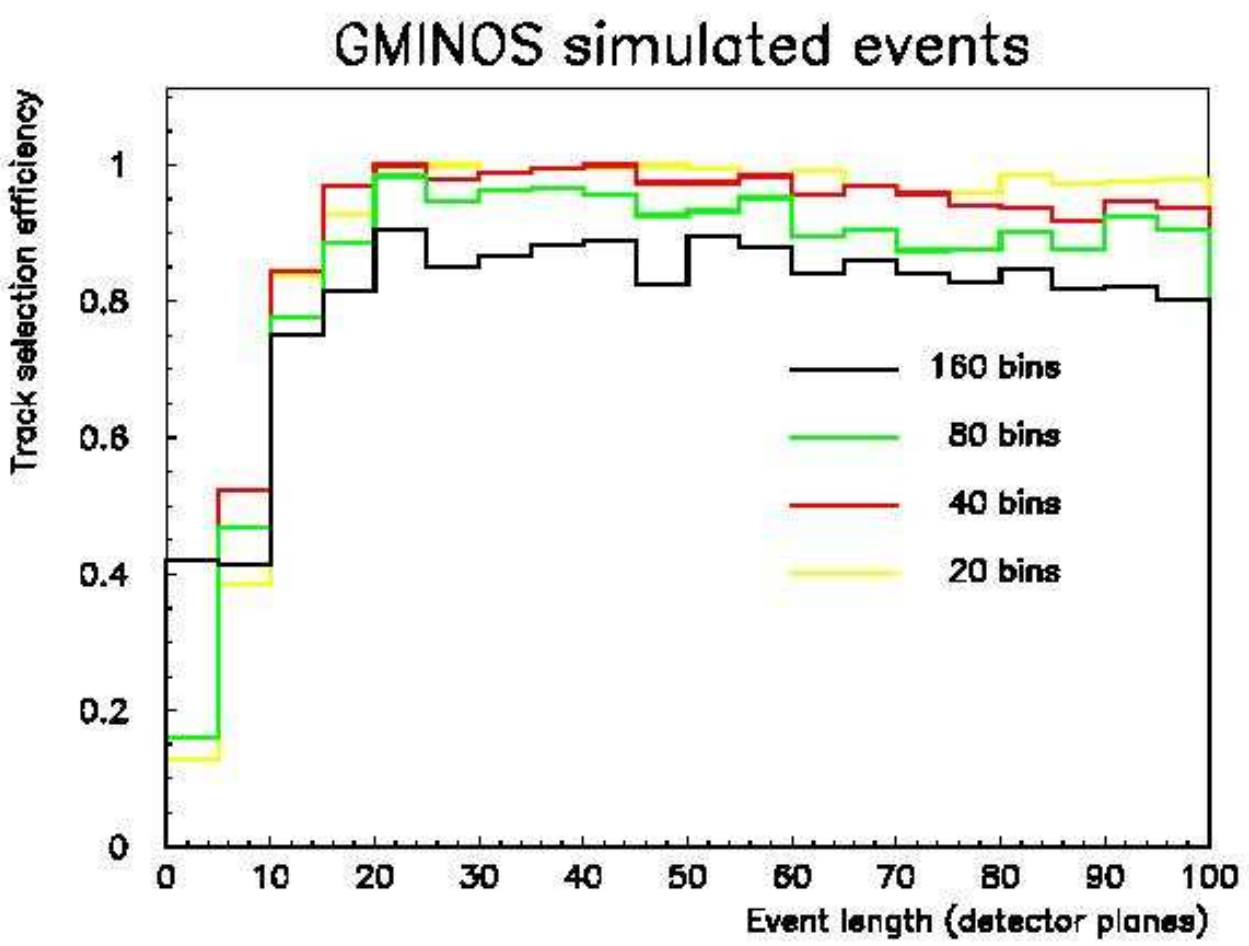

Figure 4.19: The track finding efficiency versus event length for MC for several different Hough space bin sizes (D. Petyt thesis). 


\subsection{Coordinate Systems}

The software package UgliGeometry (Unified Geometry Layered Interface) builds the positions of each strip on each plane up by starting from steel plane, adding offsets of the scintillator plane, the scintillator module and finally the strip. It provides a means to convert from logical electronics channel to geometrical position in space. The reconstruction uses UgliGeometry, and it is intended that the Monte Carlo would as well, but so far the fortran based simulations instead use the nominal, or "perfect" geometry, with all planes identical and all strip centers exactly $4.1 \mathrm{~cm}$ apart from each other. UgliGeometry by default comprises the local coordinate system.

- Ideal Geometry - that used in the original specifications and in the Monte Carlo. Currently it is much like the real detector, but with no gaps between modules or strips; ie they are perfectly made and placed.

- The Used UgliGeometry - the software package and the alignment constants of the offsets of strip and module transverse offsets. The $\mathrm{Z}$ positions of the planes are based on measurements of the total lengths of each SM of the real detector, divided by the number of planes in each SM; leading to one number for the pitch of the planes in $\mathrm{Z}$ for each SM.

- The Unused UgliGeometry - the software has the ability to also handle rotations of a strip in both the transverse (XY) and longitudinal (Z) planes. An average number for the rotation of the scintillator planes in each SM in each view has been added to the database, but the reconstruction software as of release R1.18 does not currently use this.

- The Real Geometry - there are possible misalignments in a typical strip in 
the $\mathrm{z}$ position and possible strip and module curvature which are have not been implemented.

The MINOS Far Detector uses a local coordinate system which is surveyed and linked to a global coordinate system to allow determination of the detector's alignment with the NuMI beam. The local system is aligned with survey monuments that were placed and measured in positions about the detector hall by surveyors from the Fermilab Alignment and Metrology Group and referenced by them to a global system on the surface. Distance to surface is needed to know the overburden and the alignment study gave a value of 710.25 meters for the elevation to the surface at the head frame parking lot from the floor of the MINOS cavern. The local coordinate system is shown in Figure 4.20, wherein the floor of the 27th level of the mine was linked through inertial survey to the CORS national network to provide NGS (National Geodetic Survey) high accuracy geodetic coordinates [29] of the underground hall. The global coordinates then are the longitude, latitude and altitude of the detector hall, and the local coordinates are the $\mathrm{x}, \mathrm{y}$ and $\mathrm{z}$ axes centered within the detector itself.

As shown in the Figure 4.20, the x-axis points generally West and is centered midway between the rails at the center of the coil hole. The y-axis is the local vertical and is centered a bit below the detector support rails also at the center of the scintillator/coil hole, and the z-axis points longitudinally along the detector at 26.5548 degrees West of North, centered at the south face of plane 0. The definitions of the direction cosines as well as local azimuth and zenith are defined in Figure 4.21 to be the typical astronomical coordinates referring to the origin of a track. In later chapters, terms such as direction sine and direction cotangent will be used, which is merely the sine and cotangent respectively of the inverse cosine of the direction 


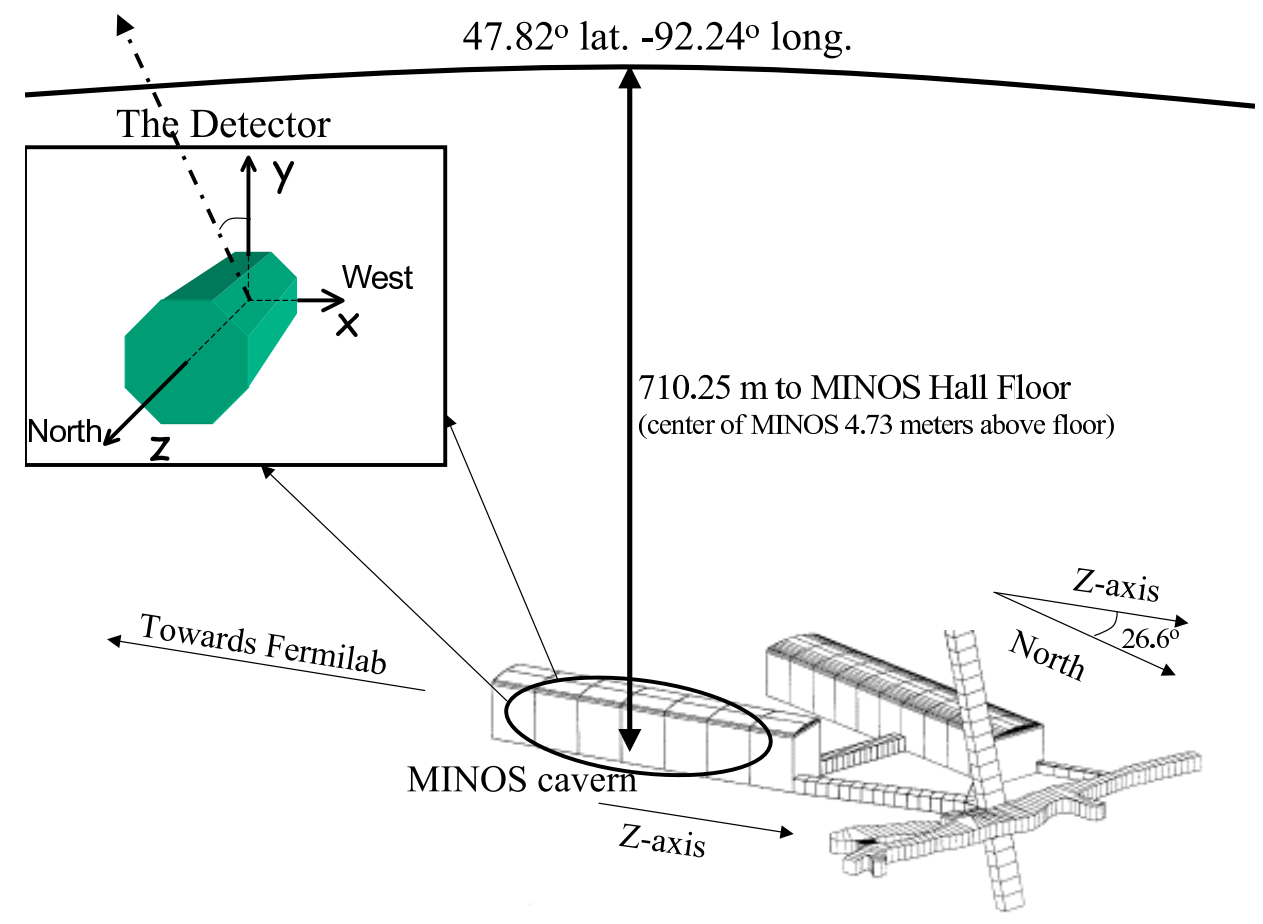

Figure 4.20: The Far Detector Hall, referenced to the surface. Inset is rotated about y-axis to show face as seen from Northern hall entrance. 


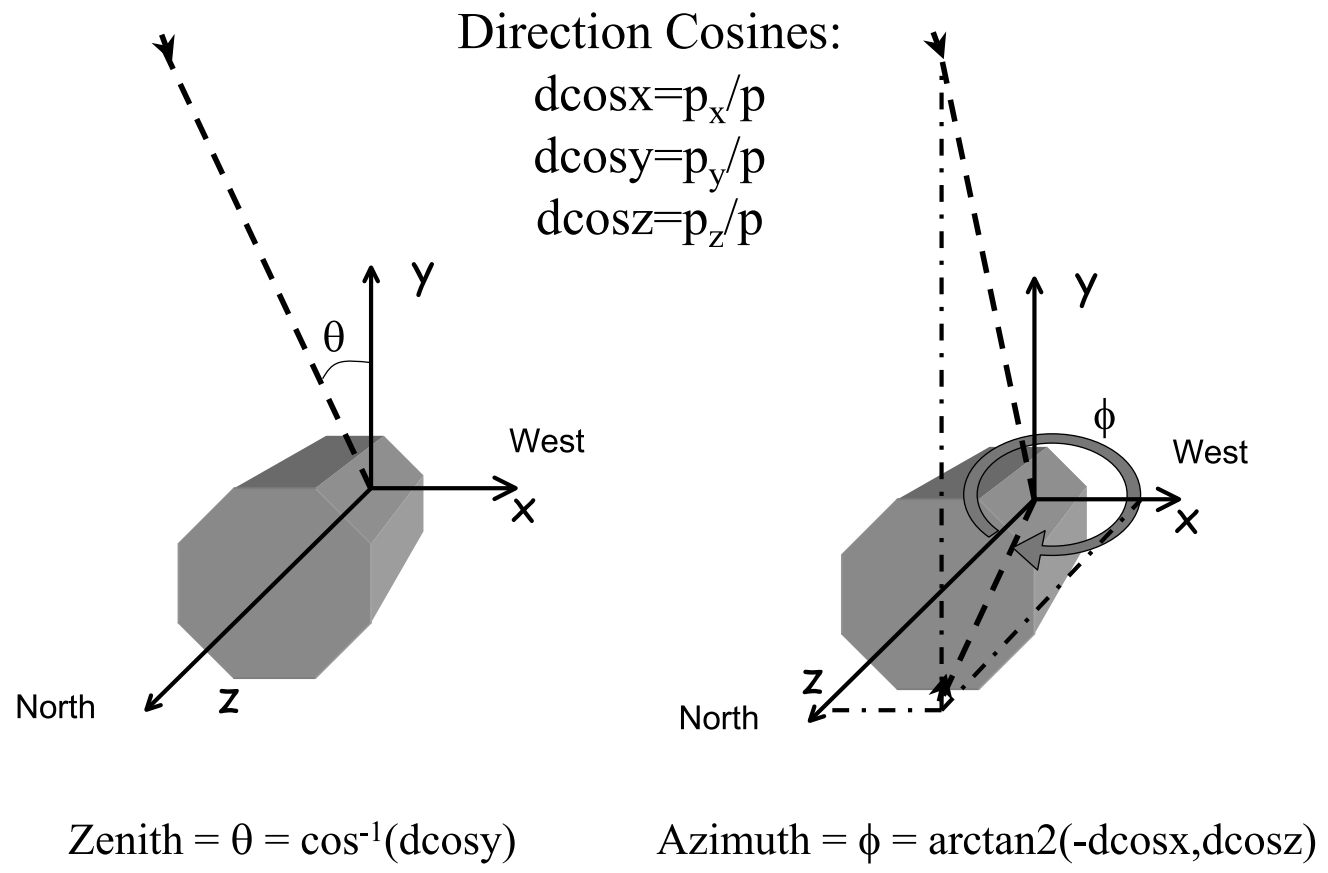

Figure 4.21: The Far Detector azimuth and zenith coordinates, as defined by the direction cosines of the track. Direction cosines are the components of the track's initial vector in the three axes. 


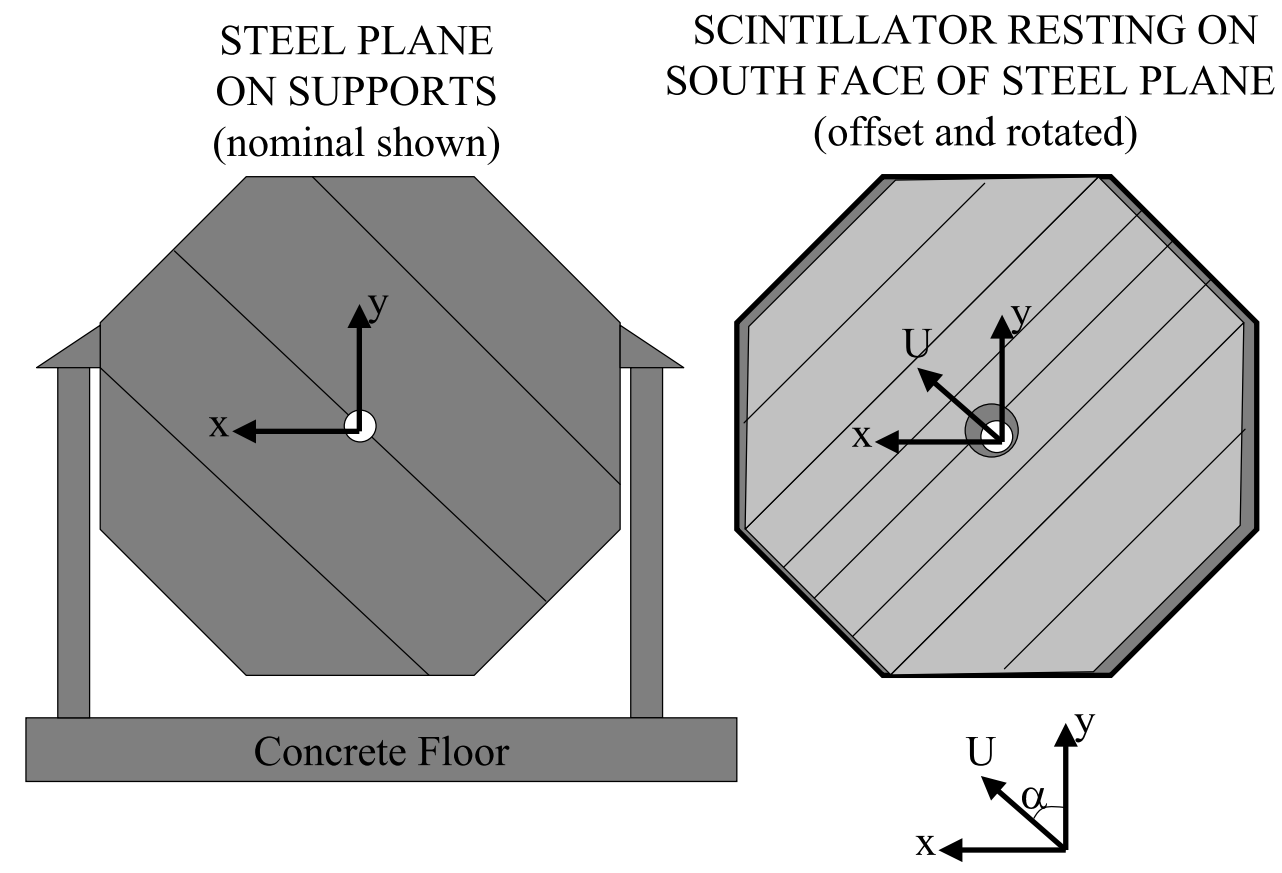

Figure 4.22: The left image is that of steel resting on supports, the center of plane is nominally set to the center of the xy-axes. The right image is that of scintillator modules affixed to a steel plane, the center of which is nominally set to the same center as the steel plane. Ultimately, the scintillator plane may be rotated and/or offset from the center of the xy-axes. 


\section{Each Module has Transverse} Position from Center in U/V axes:

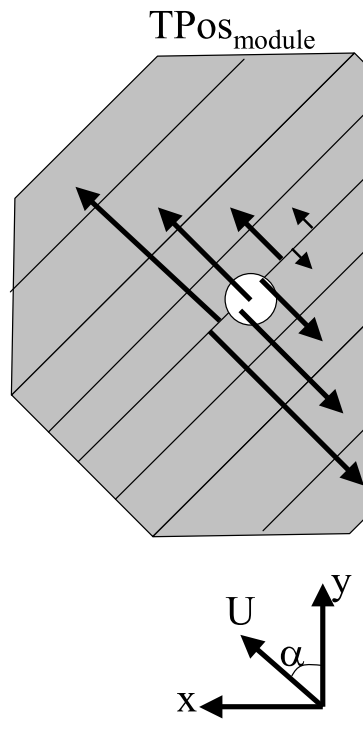

Each Strip has Transverse Position from Center of its module: TPos $_{\text {strip }}$

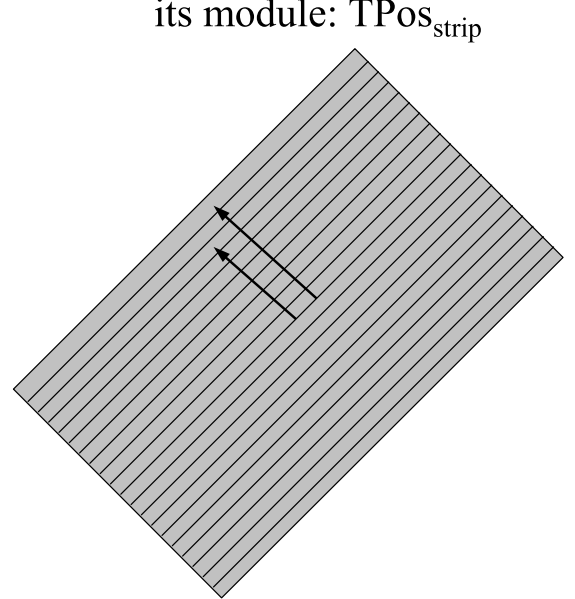

By adding $\mathrm{TPos}_{\text {strip }}$ to $\mathrm{TPos}_{\text {module }}$ to offset of scintillator plane to offset of steel plane, "UgliGeometry" of U,V positions is built.

Figure 4.23: The left image is of scintillator modules on a plane, and the right is of scintillator strips within a module, each of which has a transverse position in the database to the strip's center. 
cosine referred to. These components will all begin with "d" for direction, and not differential, component such as dcosz for the direction cosine meaning the track's component in the z-axis.

In Figure 4.22, the position of a steel and scintillator plane is shown with respect to the local coordinate system, and in Figure 4.23, the positions of each of the eight modules w.r.t. a steel plane, and also the positions of each strip within one of the modules w.r.t. the module's center position is shown. The coordinate system used by the reconstruction software refres to $\mathrm{U}$ and $\mathrm{V}$ axes, which are at $45^{\circ}$ to the XY-axes. As in Figure 4.23 , the position of a strip in $\mathrm{U}$ or $\mathrm{V}$ is built up in the MINOS geometry by adding the database values of the following together: the strips transverse position from the center of a module, the module's transverse position from the center of the scintillator plane, the scintillator plane's offset from the center of the steel plane, and the steel plane's offset from the center of the coordinate system. In the MINOS database, the scintillator plane and the steel plane's transverse offsets are set to zero, and only the module's and strips transverse positions have moved from nominal. The strips positions within modules are set from the "mapper" data, which is comprised of data taken from running a radioactive source accurately with stepper motors over a face of the modules and recording the light output. The module transverse positions are taken from the muon alignment study, which uses residuals of straight line fits to muon tracks in the detector to set module positions as a groups of strips [30].

\subsection{Typical Tracks}

Note the sizes of the sagitta in each case. Very similar sagitta are calculated in Table 5.5 ignoring multiple scattering and energy loss. Charge determination is a function of dcosz and impact parameter, as will be seen in the next chapter. 

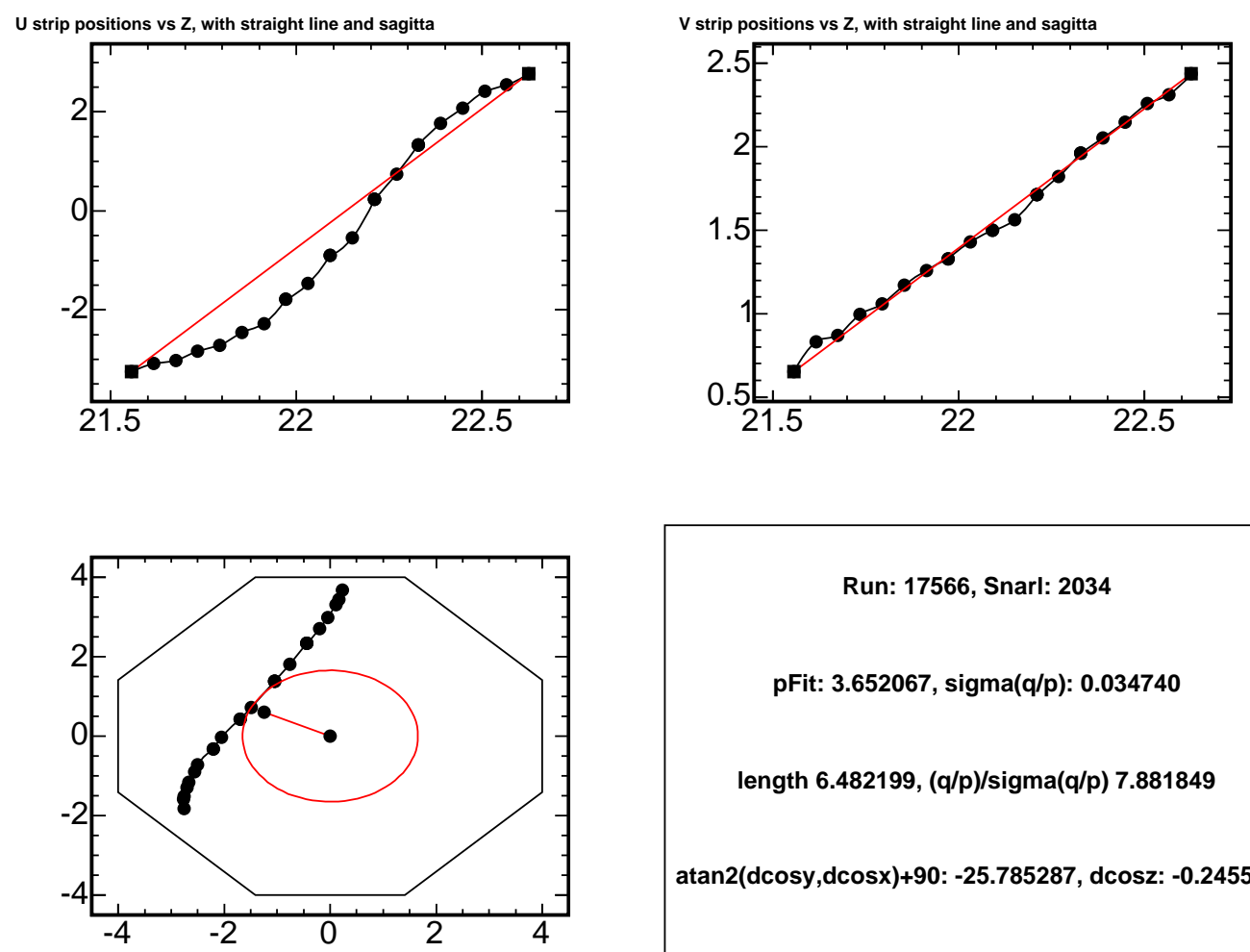

Run: 17566, Snarl: 2034

pFit: $3.652067, \operatorname{sigma(q/p):~} 0.034740$

length 6.482199, (q/p)/sigma(q/p) 7.881849

atan2(dcosy,dcos $x)+90:-25.785287, d \cos z:-0.245580$

U sagitta: $0.181317, \mathrm{~V}$ sagitta: 0.041564

Figure 4.24: A low energy cosmic muon track with impact parameter of 2 meters in the MINOS Far Detector. 

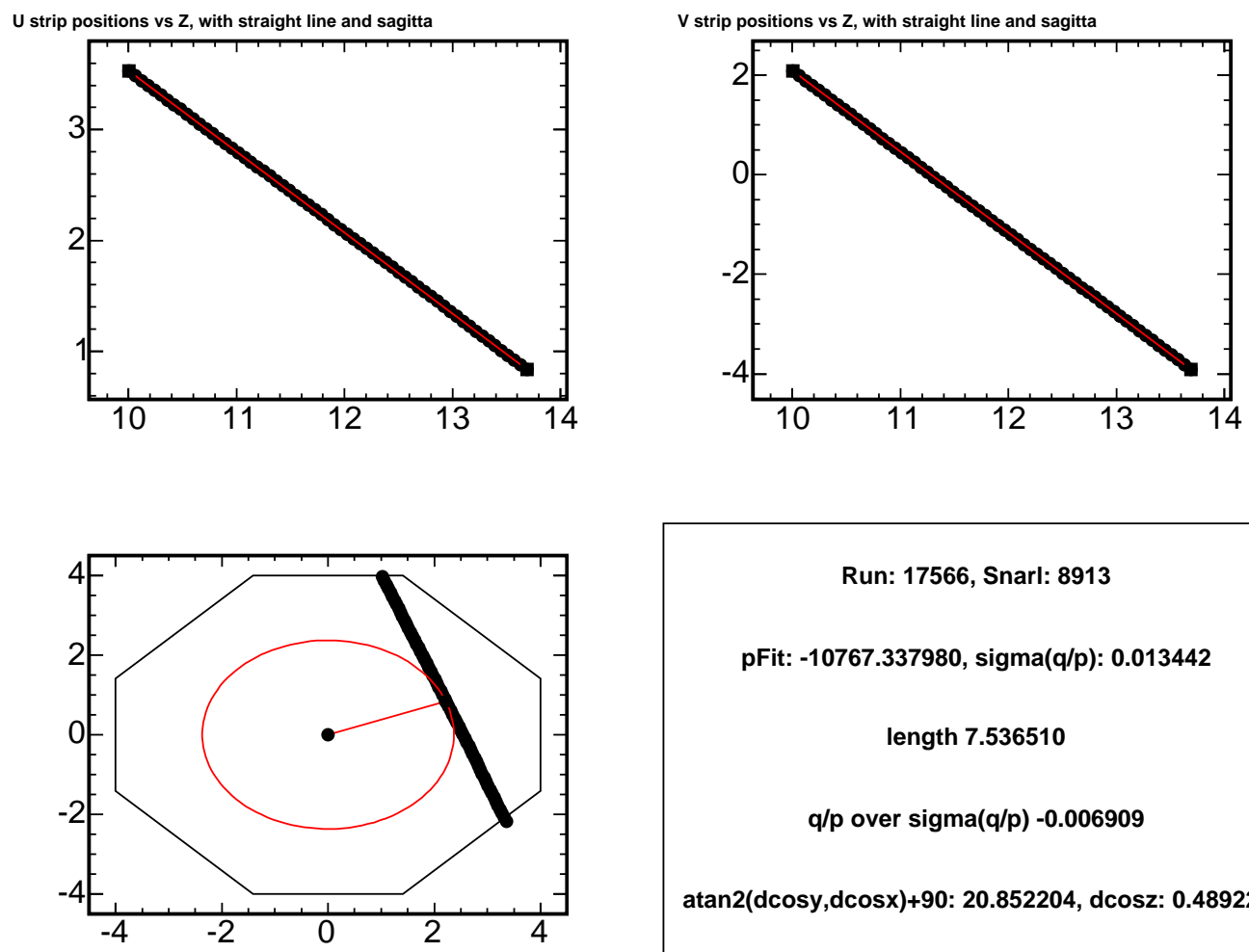

Run: 17566, Snarl: 8913

pFit: -10767.337980, sigma(q/p): 0.013442

length 7.536510

$q / p$ over sigma(q/p) -0.006909

atan2(dcosy,dcos $x)+90: 20.852204, d \operatorname{cosz}: 0.489222$

U sagitta: $0.008143, \mathrm{~V}$ sagitta: 0.005559

Figure 4.25: A high energy, straight muon track in the MINOS Far Detector. 

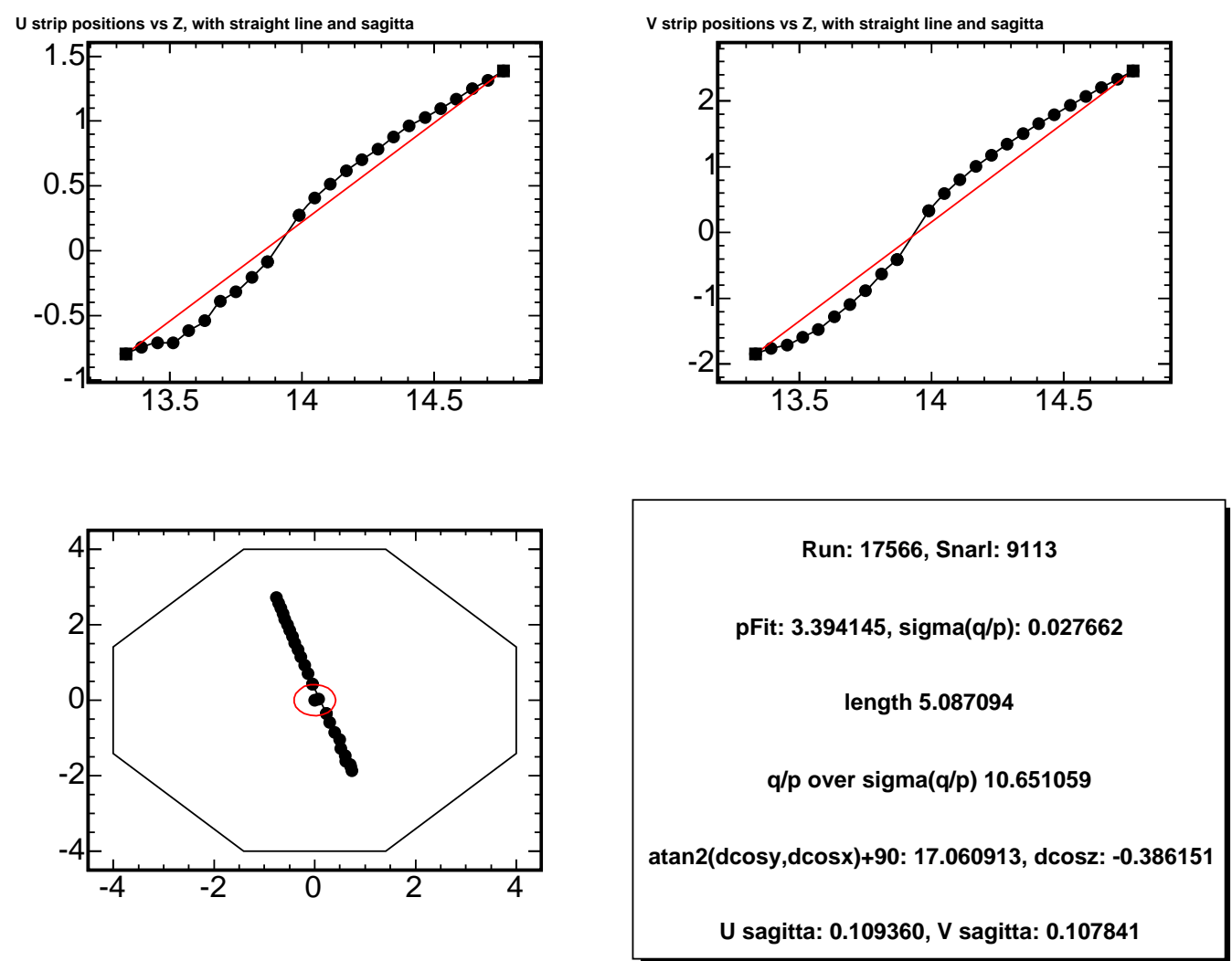

Figure 4.26: A low energy muon track in the MINOS Far Detector traversing the coil hole. 

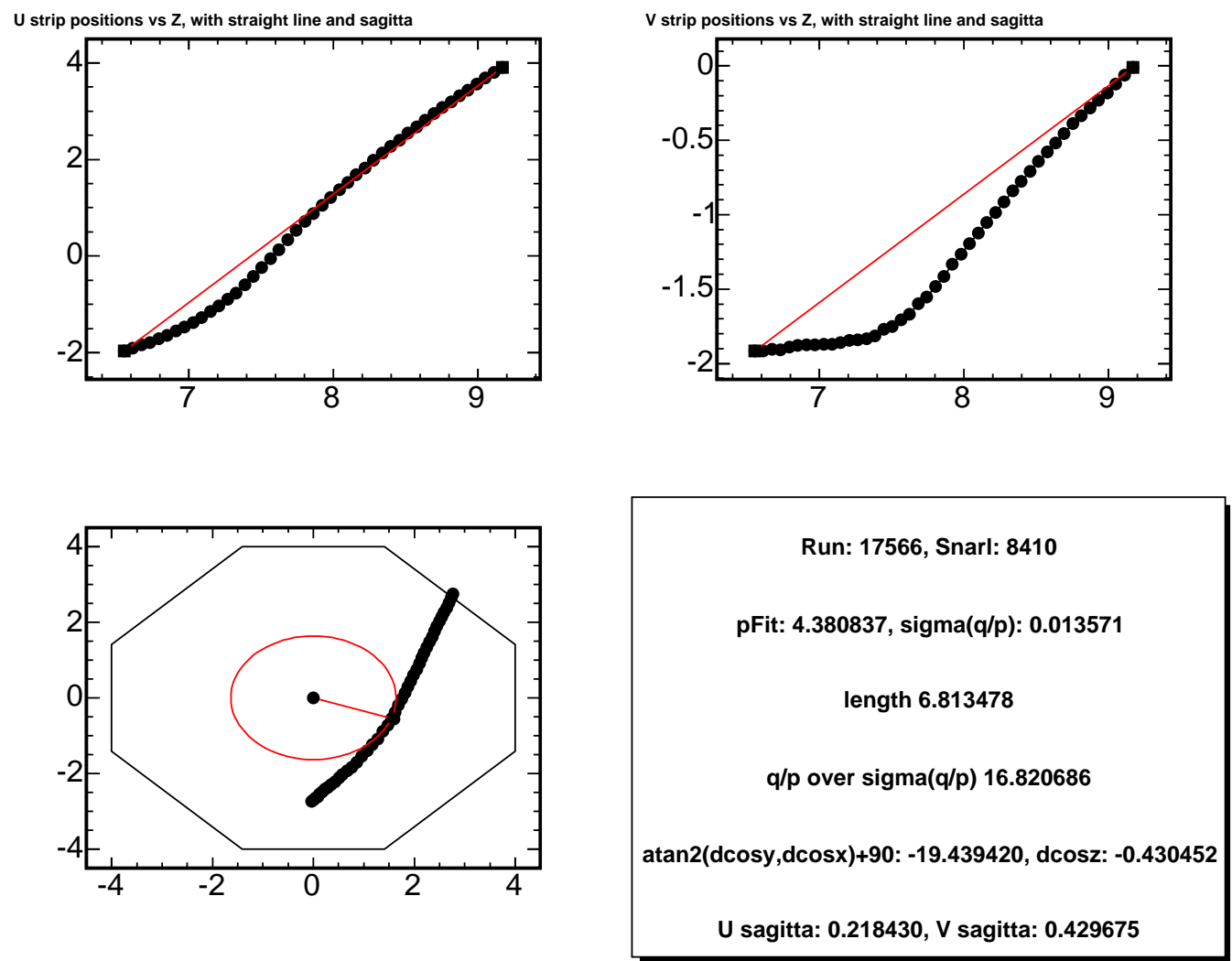

Figure 4.27: A low energy, stopping muon track in the MINOS Far Detector. 


\section{Chapter 5}

\section{Cosmic Ray Muons in MINOS}

\subsection{Data and Monte Carlo Cuts}

The cuts used in this analysis proceeded from work done by Brian Rebel [7]. There are fiducial cuts, track quality cuts, and a curvature resolution cut. The effect of the cuts on each of the two data samples is summarized in Table 5.1 for the Forward Field Data sample, and in Table 5.2 for the Reverse Field Data sample. The effect on the Monte Carlo has been listed for both Forward and Reverse Field samples in Table 5.3.

The cuts with the largest effects on the samples are the 20 plane cut, the reduced $\chi^{2}$ cut, the UV asymmetry cut, and the QPID cut. There is also one cut considered apart from the other cuts which removes entire DAQ runs that had a rate that deviated more than $3 \sigma$ from the average rate determined for the month that the run was taken from. The effect of this cut, termed the run rate cut, is mainly to remove false data from the light injection calibration process that made it into the data stream despite the design to keep this calibration data separate, a problem which has since been fixed in the DAQ software. In the data plots, a peak at an azimuth of 153.5 degrees is visible before this cut is made, indicating light injected into the scintillator manifolds is being reconstructed as tracks coming directly from Fermilab. Due to electronics deadtime being a greater issue when all the PMTs in a 


\begin{tabular}{|l|c|c|}
\hline Data Cut & Number of Events & Percent Remaining \\
\hline \hline None & $16,161,396$ & 128.57 \\
\hline$\Delta_{\text {Run Rate }}<3 \sigma$ & $12,570,419$ & 100.00 \\
\hline 20 Planes in Track & $9,260,333$ & 73.67 \\
\hline 2 Meter Track Length & $9,208,456$ & 73.25 \\
\hline Reduced $\chi^{2}<2.5$ & $8,617,295$ & 68.55 \\
\hline Passes Track Fitter & $8,596,681$ & 68.39 \\
\hline Passes Demux & $8,590,358$ & 68.34 \\
\hline Non-Contained Vertex & $8,583,165$ & 68.28 \\
\hline Nonphysical Vertex/End & $8,552,061$ & 68.03 \\
\hline Downgoing Direction & $8,551,329$ & 68.03 \\
\hline U/V Plane Asymmetry & $8,123,871$ & 64.63 \\
\hline Plane and Pulse Height Usage & $8,114,992$ & 64.56 \\
\hline QPID $\frac{\sigma(q / p)}{q / p}<40 \%$ & $2,254,494$ & 17.93 \\
\hline
\end{tabular}

Table 5.1: Effect of Incremental Cuts on Forward Field Data Sample.

small region are being illuminated, these fake tracks do not necessarily reconstruct as coming directly directly to or from Fermilab, but only show a distinct tendency to do so. The run rate cut is very effective at removing these events since they were also localized in time.

\subsection{Detector Acceptance and Efficiency}

The detector response to incident muons as a function of incident angles must be understood from Monte Carlo simulations. The response of the detector is examined in the local azimuth and zenith incident muon angles. Acceptance for a bin of azimuth, zenith is the efficiency of that same bin times projected area and solid angle for that bin of azimuth, zenith. Efficiency for a bin of azimuth, zenith is defined as the proportion of Monte Carlo events passing some trigger and quality cuts out of the total number of events generated in that bin. A GEANT-based Monte Carlo simulation was developed for the MINOS detectors that included many effects including the rock above the detector, energy loss and multiple scattering in 


\begin{tabular}{|l|c|c|}
\hline Data Cut & Number of Events & Percent Remaining \\
\hline \hline None & $8,717,439$ & 119.55 \\
\hline$\Delta_{\text {Run Rate }}<3 \sigma$ & $7,534,356$ & 100.00 \\
\hline 20 Planes in Track & $5,366,993$ & 73.60 \\
\hline 2 Meter Track Length & $5,336,865$ & 73.19 \\
\hline Reduced $\chi^{2}<2.5$ & $4,996,171$ & 68.52 \\
\hline Passes Track Fitter & $4,984,624$ & 68.36 \\
\hline Passes Demux & $4,980,997$ & 68.31 \\
\hline Non-Contained Vertex & $4,977,080$ & 68.26 \\
\hline Nonphysical Vertex/End & $4,959,179$ & 68.01 \\
\hline Downgoing Direction & $4,958,845$ & 68.01 \\
\hline U/V Plane Asymmetry & $4,713,601$ & 64.64 \\
\hline Plane and Pulse Height Usage & $4,708,470$ & 64.57 \\
\hline QPID $\frac{\sigma(q / p)}{q / p}<40 \%$ & $1,290,566$ & 17.70 \\
\hline
\end{tabular}

Table 5.2: Effect of Incremental Cuts on Reverse Field Data Sample.

\begin{tabular}{|l|c|c|c|c|}
\hline MC Cut & \# Reverse & \% Left & \# Forward & \% Left \\
\hline \hline None & $1,018,328$ & 100.00 & $1,015,668$ & 100.00 \\
\hline 20 Planes in Track & 771,761 & 75.79 & 769,897 & 75.80 \\
\hline 2 Meter Track Length & 766,723 & 75.29 & 764,901 & 75.31 \\
\hline Reduced $\chi^{2}<2.5$ & 735,891 & 72.26 & 733,678 & 72.24 \\
\hline Passes Track Fitter & 735,433 & 72.22 & 733,226 & 72.19 \\
\hline Passes Demux & 735,397 & 72.22 & 733,182 & 72.19 \\
\hline Non-Contained Vertex & 734,984 & 72.18 & 732,758 & 72.15 \\
\hline Nonphysical Vertex/End & 734,120 & 72.09 & 731,949 & 72.07 \\
\hline Downgoing Direction & 734,107 & 72.09 & 731,941 & 72.07 \\
\hline U/V Plane Asymmetry & 718,681 & 70.57 & 716,489 & 70.54 \\
\hline Plane and Pulse Height Usage & 718,338 & 70.54 & 716,113 & 70.50 \\
\hline QPID $\frac{\sigma(q / p)}{q / p}<40 \%$ & 232,240 & 22.81 & 230,908 & 22.73 \\
\hline
\end{tabular}

Table 5.3: Effect of Incremental Cuts on Monte Carlo Sample. 
the detector, optical crosstalk, PMT response, bremsstrahlung (not tuned), light transport from hit to PMT, DAQ triggers, the magnetic field map and the same reconstruction chain as the data. The required cuts for a track were all the cuts used in the data analysis cuts except for the run rate cut and the $(q / p) / \sigma(q / p)$ cut.

$$
\begin{gathered}
\epsilon_{\phi, \theta}=\frac{N_{\phi, \theta}(\text { passing cuts })}{N_{\phi, \theta}(\text { no cuts })} \\
\text { Acceptance }_{\phi, \theta}=\epsilon_{\phi, \theta} \times \text { Area }_{\phi, \theta} \times \Omega
\end{gathered}
$$

\subsubsection{Monte Carlo Generation}

Monte Carlo events were generated in the following numbers: 1 million muon events were propagated through the detector in the Forward field configuration and 1 million events were propagated with a Reversed field. These were then summed together. Using Monte Carlo and Data with the Forward field case only is explored in Rebel's thesis [7]. The muons used to illuminate the detector follow a distribution generated to give an intensity that matches that expected based on the Soudan 2 overburden rock map, to be discussed later.

In Figure 5.1, the MINOS detector's efficiency is plotted as a function of azimuth and zenith. Efficiency was calculated by making all the aforementioned cuts except the QPID cut (to be discussed later). The projected area is shown in Figure 5.3, and the product of these and the solid angle is shown as the acceptance in Figure 5.4. The projected area has dips of roughly tens of percent depending on the azimuth and zenith, while the efficiency and hence the acceptance drop completely to zero for certain regions of azimuth and zenith. In particular, no cosmic muon events were expected to be up-going, so none were simulated, and so for dcosy $<0.2$ no generated events translates to no calculated efficiency and zero acceptance. Finally, 
the azimuth angles that project a track perpendicular to the detector's z-axis also have small acceptance, because efficiency in making a good track is dependent upon passing the 20 plane cut, so tracks perpendicular to the z-axis are unlikely to cross 20 planes.

For a particular field configuration, there are regions where the efficiency depends strongly on muon charge. This charge-dependent efficiency is shown most clearly in Figures 5.6 and 5.7. It is finally removed through summing the Forward and Reverse Monte Carlo sets in Figure 5.8.

\subsection{The Data}

The Far Detector DAQ system was set to record the hits inside the detector over "Runs", which lasted between several minutes and 24 hours. Occasionally conditions in the detector change significantly during a Run, for example, high voltage on a series of PMTs may drop out or more importantly one of the two magnet coils may trip off, creating an unknown magnetic field in the detector. Each Run was examined by the RunSummary software by Ben Speakman to ensure there were no high voltage trips, and verified that the magnetic field was in stable configuration for the duration of the run. The raw data for each Run was collected and processed on the portion of the Fermilab batch processing system reserved for MINOS and the output was stored on tape as ntuples containing information about reconstructed tracks. The data Runs examined are listed in Table 5.4, with their respective numbers of events and field configurations detailed. Events from SM1 and SM2 Only Field Off sets were confined to those Runs with no field in their respective Supermodules and events refer to snarls with at least one track found and fit.

The data was taken in three different configurations: 


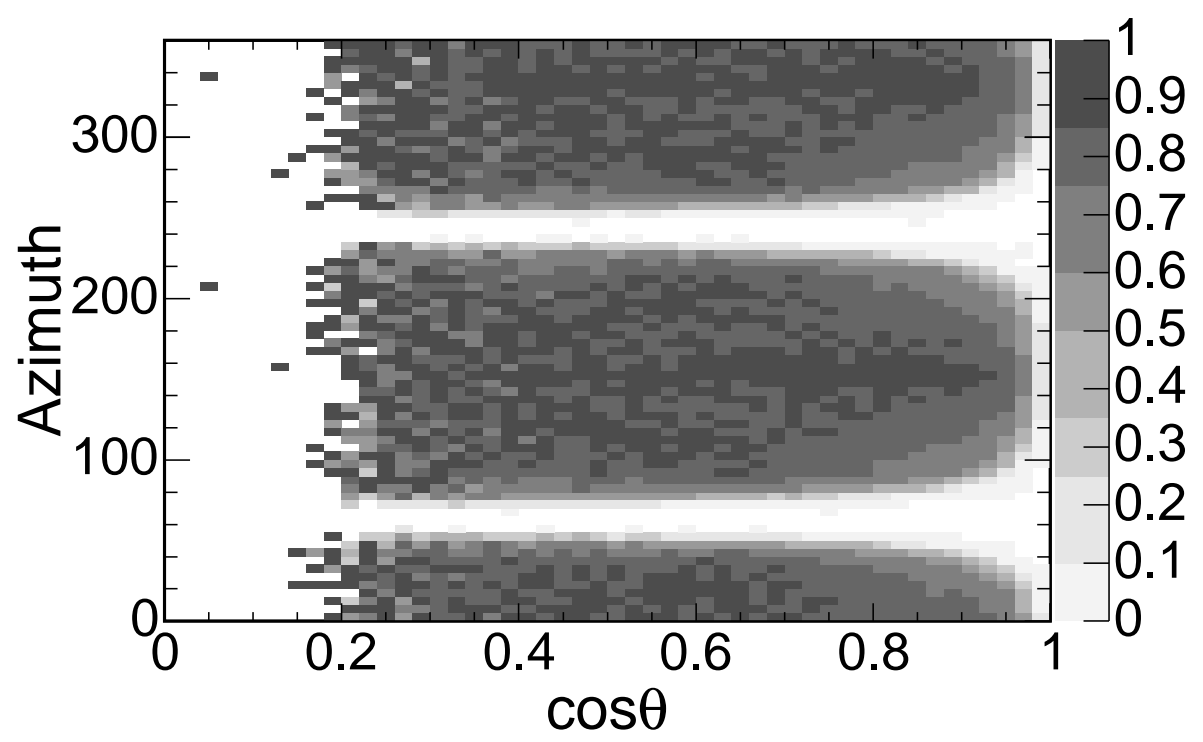

Figure 5.1: The efficiency $\epsilon$ of the analysis cuts minus the QPID cut for the MINOS Far Detector as a function of azimuth and zenith.

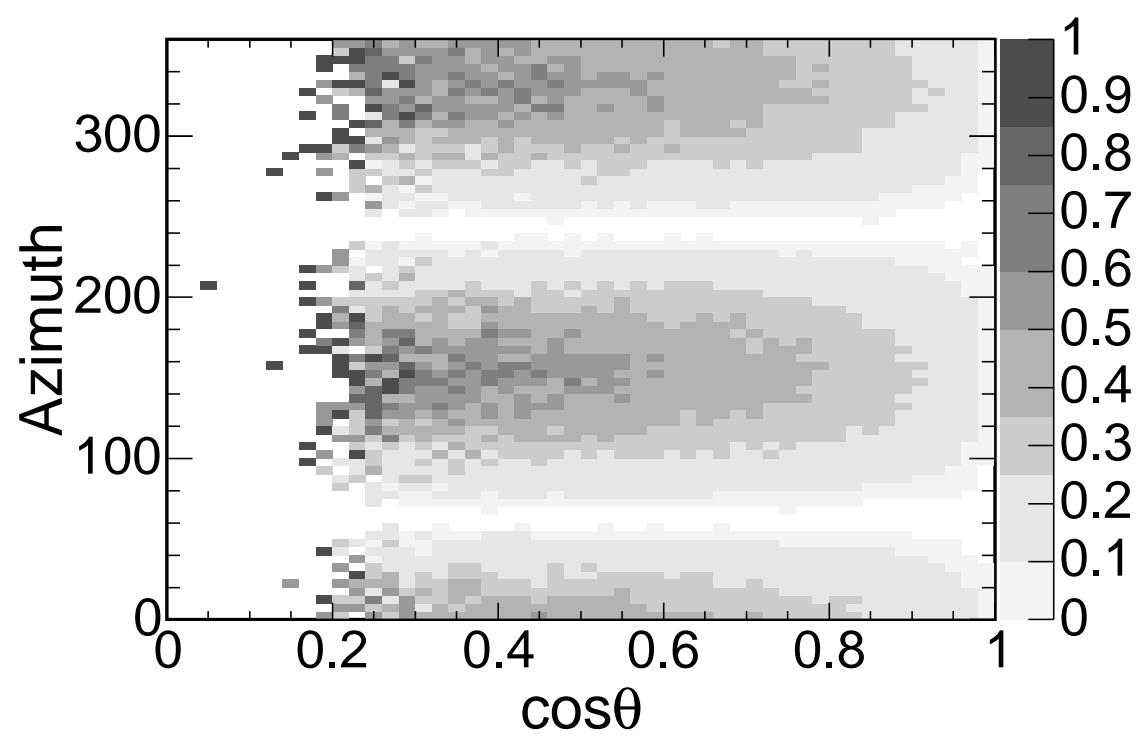

Figure 5.2: The efficiency $\epsilon$ of the analysis cuts plus the QPID cut for the MINOS Far Detector as a function of azimuth and zenith. 


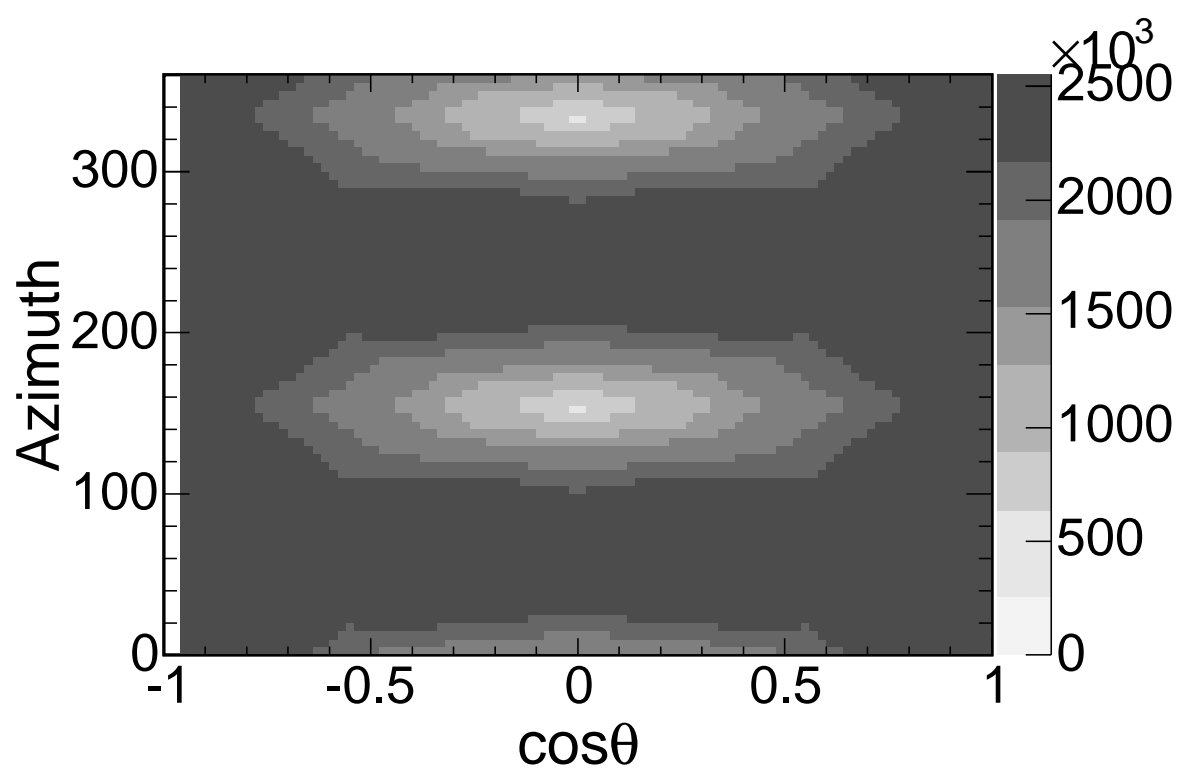

Figure 5.3: The calculated projected area of the MINOS Far Detector in $\mathrm{cm}^{2}$ as a function of azimuth and zenith.

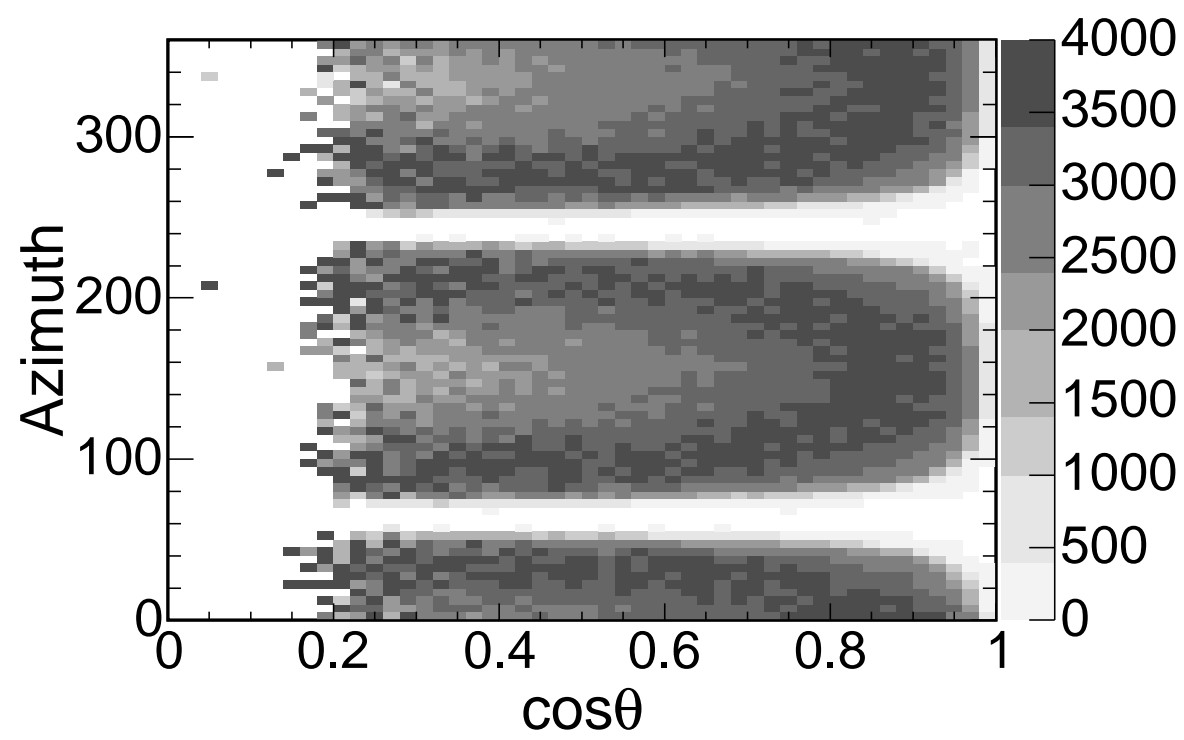

Figure 5.4: The MINOS Far Detector's Monte Carlo acceptance in $\mathrm{cm}^{2} s r$ of muons passing all cuts except the QPID cut as a function of azimuth and zenith. 


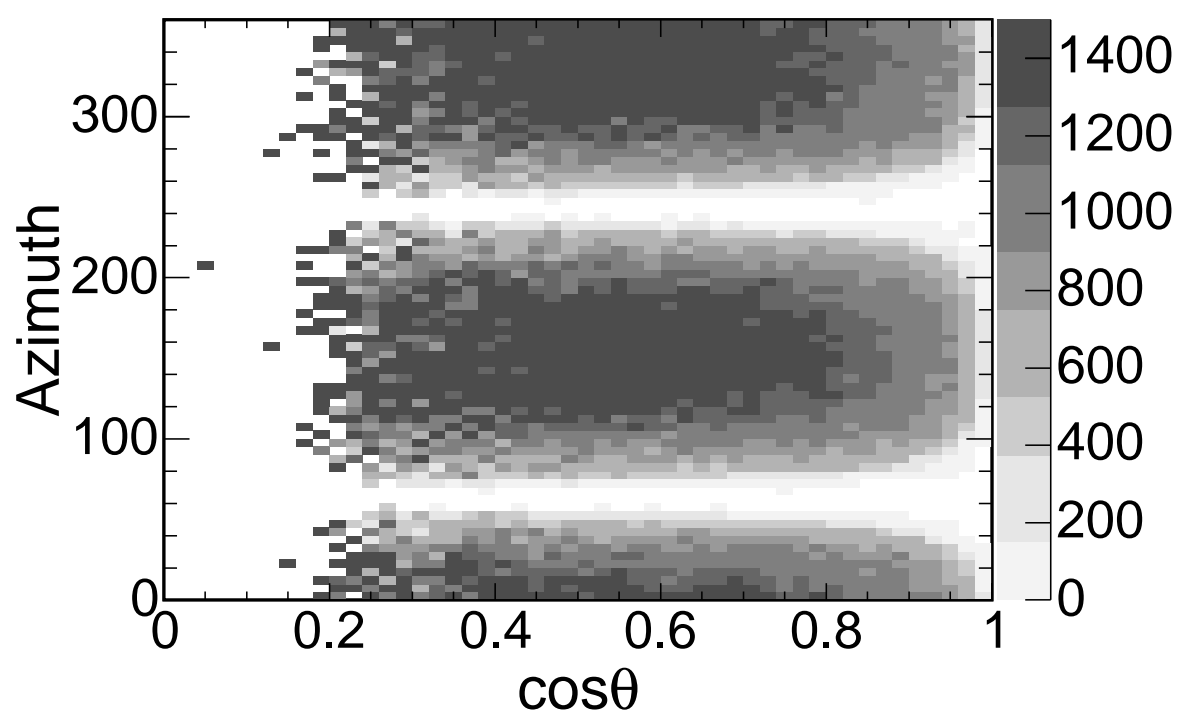

Figure 5.5: The MINOS Far Detector's Monte Carlo acceptance in $\mathrm{cm}^{2} s r$ of muons passing all analysis cuts including the QPID cut as a function of azimuth and zenith.

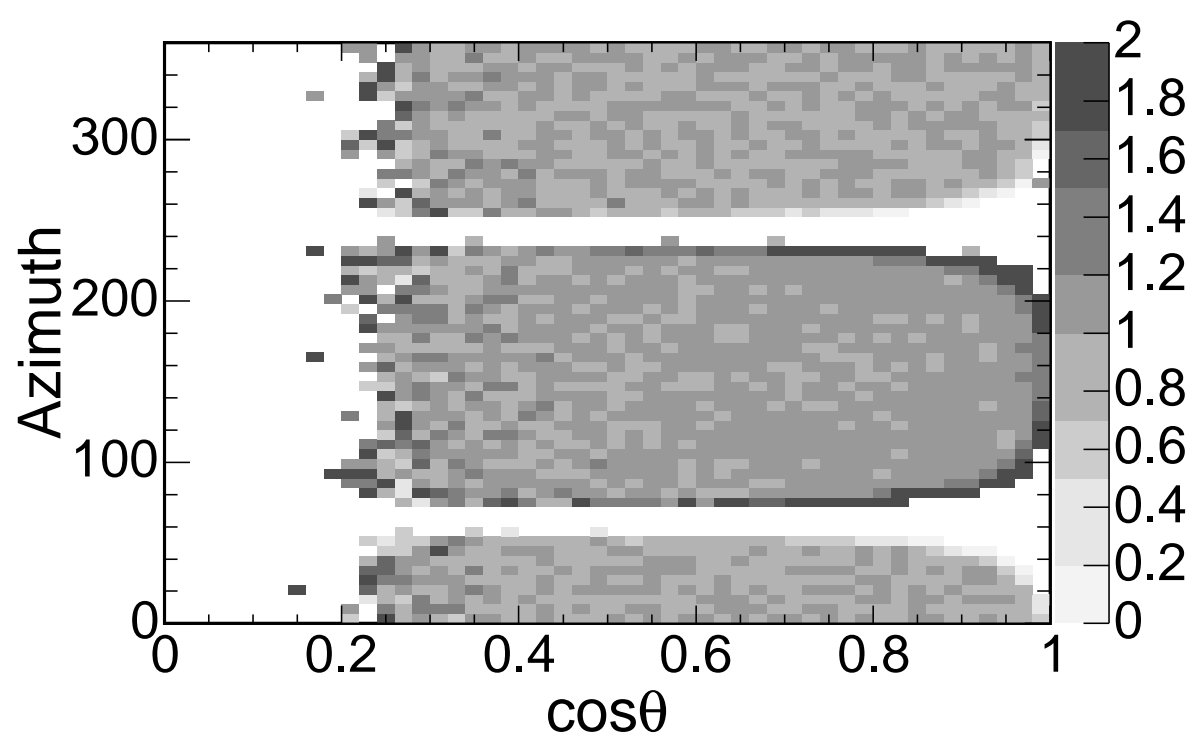

Figure 5.6: The ratio of the efficiency of positive over negative muon reconstruction in the Forward Field MC, as a function of azimuth and zenith. Efficiency at angles close to perpendicular to z-axis prefers positives for North-going muons and negatives for South-going muons. 


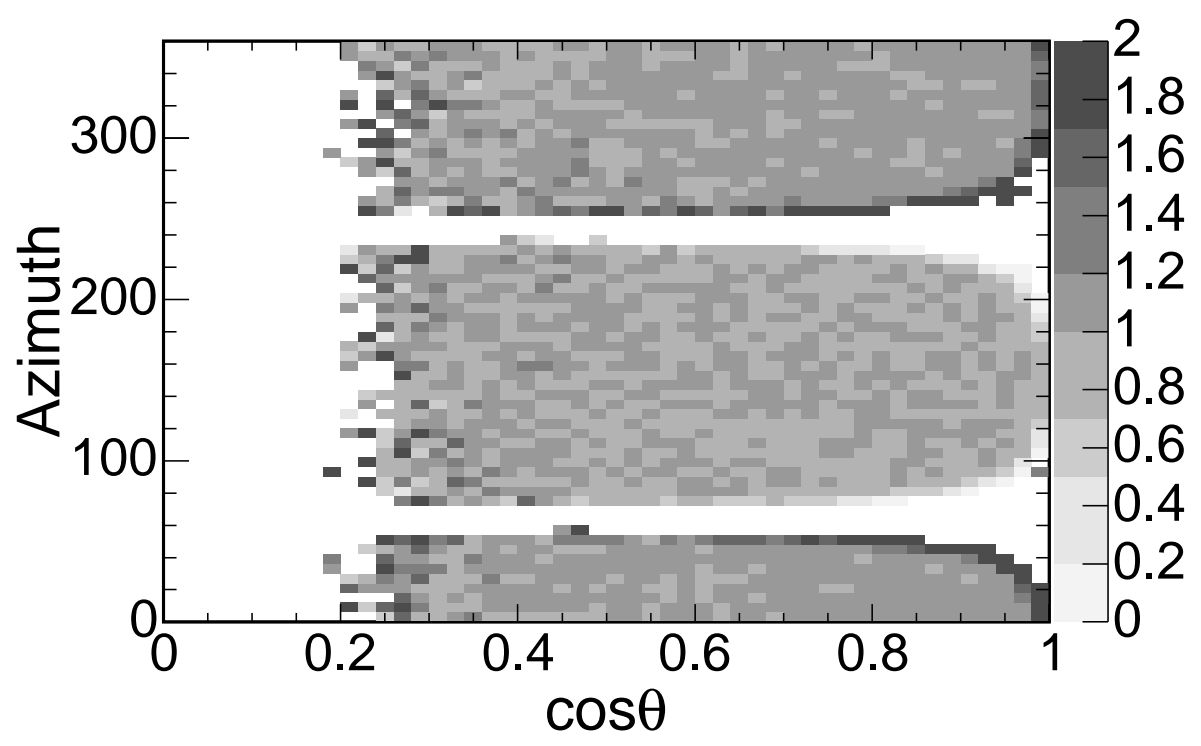

Figure 5.7: The ratio of the efficiency of positive over negative muon reconstruction for Reverse Field MC, as a function of azimuth and zenith. Efficiency at angles close to perpendicular to z-axis prefers negatives for North-going muons and positives for South-going muons.

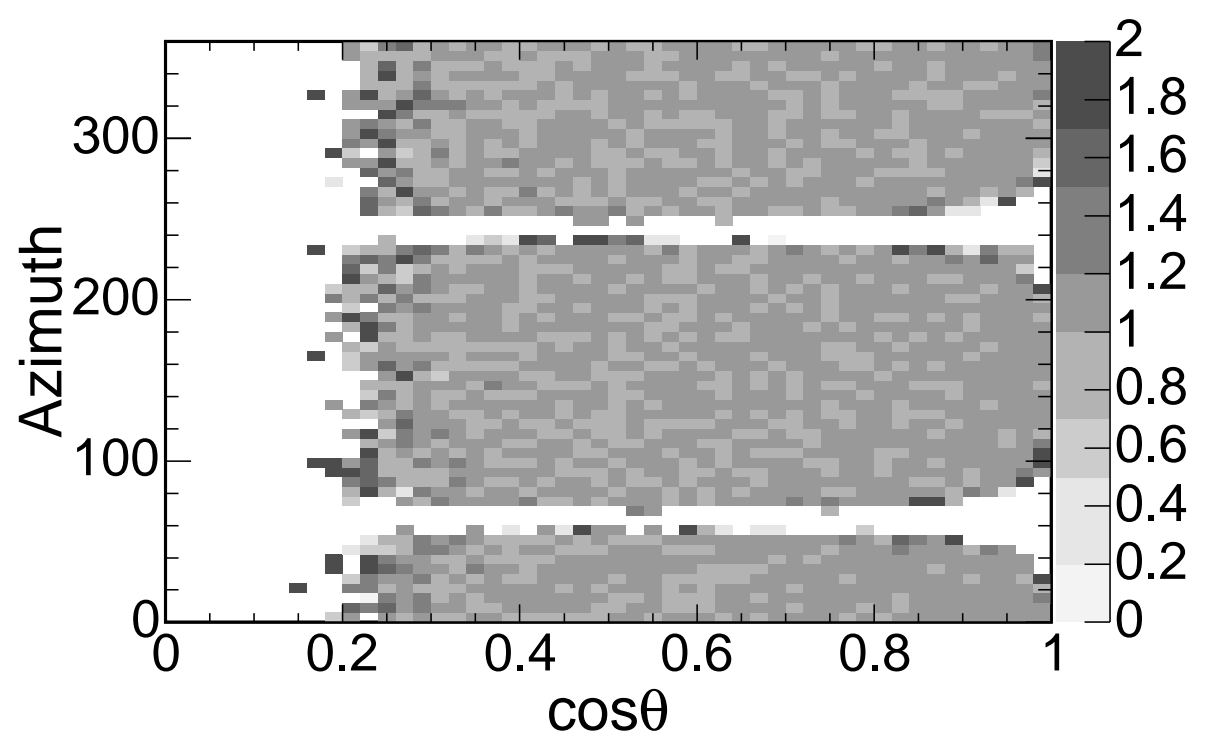

Figure 5.8: The ratio of efficiency of positive over negative muon reconstruction, after summing forward field and reverse field MC sets, as a function of azimuth and zenith. Efficiency is no longer biased to either charge due to the summing procedure. 
(1) a field off data set for each SM was taken before the magnetic coil was installed and energized for alignment purposes,

(2) the nominal field polarity was set, and Forward field data was taken,

(3) the coil current polarity was reversed (following a degaussing procedure) and Reverse field data was taken,

\begin{tabular}{|l|c|c|c|}
\hline Run Numbers & Dates & Events & Field Config \\
\hline \hline $5,963-6,497$ & June 28 - July 22, 2002 & 84,953 & SM1 Field Off \\
\hline $16,475-16,613$ & June 11-18, 2003 & 120,653 & SM2 Field Off \\
\hline $17,566-25,764$ & July 17, 2003 - June 21, 2004 & $12,772,566$ & Forward \\
\hline $25,767-29,090$ & June 21, 2004 - February 1, 2005 & $8,714,191$ & Reversed \\
\hline $29,096-31,356$ & February 1 - April 30, 2005 - & $3,392,078$ & Forward \\
\hline
\end{tabular}

Table 5.4: Data Runs and events analyzed, dates. Note that Run Number Ranges are not consecutive and may have large gaps.

The observed muon data as a function of azimuth and zenith is shown in Figure 5.9. Using Eqn 5.3 and the Monte Carlo determined acceptance from above, the intensity is plotted in Figure 5.10. Note that this intensity uses the acceptance calculated without the QPID cut, because doing so otherwise would not return the true intensity of muons underground but instead just the low energy subset that reconstructs with confidence in MINOS.

$$
I_{\mu}(\phi, \theta)=\frac{N_{\mu}(\phi, \theta)}{\Delta T \times \operatorname{Acceptance}(\phi, \theta)}
$$

The dips in azimuth are due to the 20 plane requirement, as discussed above. Next, the charge ratio as a function of azimuth is plotted in 5.11, which shows very interesting and originally unexpected structure. The muons reaching the Soudan cavern were created by primaries with energies far too high to be affected by earth's magnetic field, so the roughly $10 \%$ bias at various azimuths was very surprising. 


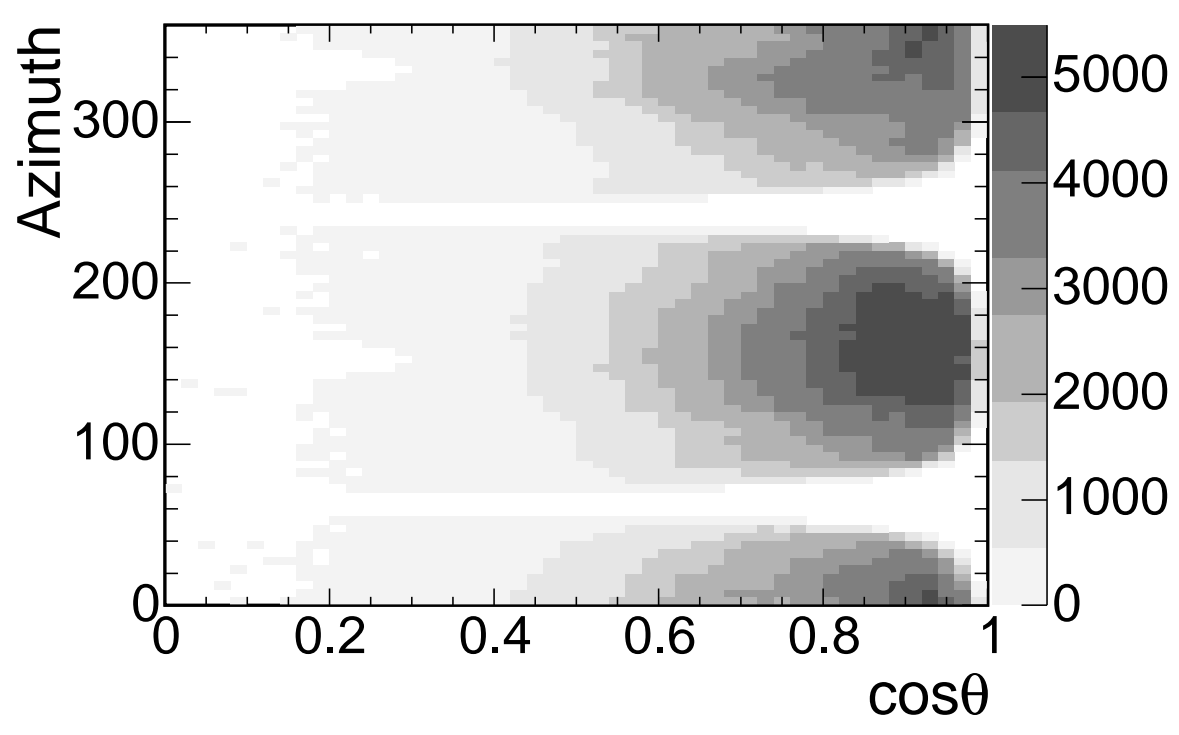

Figure 5.9: The muon data sample from July 2003 through April 2005 as a function of azimuth and zenith.

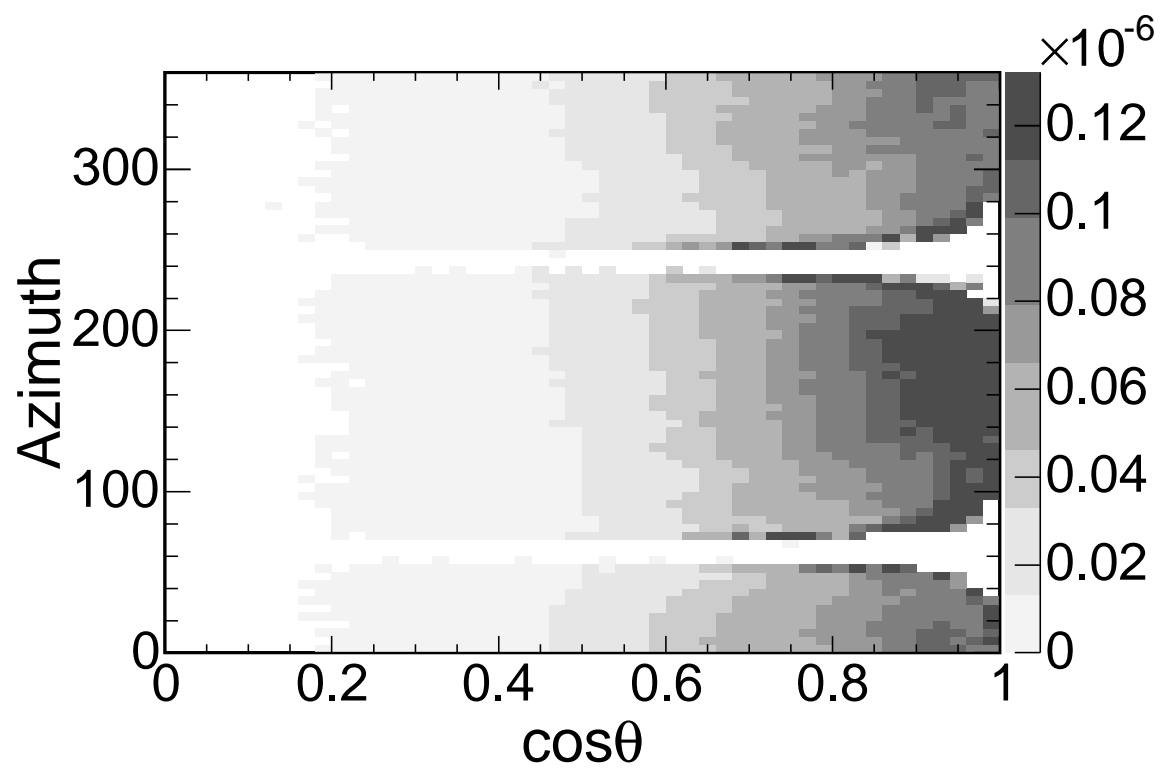

Figure 5.10: The intensity of incident (calculated without QPID cut) muons at the MINOS Far Detector as a function of azimuth and zenith. See Figure 2.7 to compare intensity near vertical $\approx 1.2 \times 10^{-7} \mathrm{~cm}^{-2} \mathrm{~s}^{-1} \mathrm{sr}^{-1}$. 
It is important to distinguish between the structure that is understood and that which is not. From the Monte Carlo plots, there are two behaviors which were initially surprising. The detector was designed to focus $\mu^{-}$from the South, since the beam would be $\nu_{\mu}$ and not $\bar{\nu}_{\mu}$. For this reason, muons from the South would be preferentially accepted as negatives. Negatives see a field which curves them towards the center of the detector, while a positive is curved outwards out of the detector, so a negative is focussed and a positive defocussed. From the North, positives would be preferentially accepted, due to this same focussing effect operating in an inverse way upon the charged muons (and so for this direction and field configuration the positive is a focussing muon, while negatives are defocussing). In the Reverse field configuration, the opposite would be true and was observed in Monte Carlo after the detector's field had been reversed. Since the muon intensity is higher from the South by approximately $10 \%$, the observed charge ratio would be affected.

The second behavior is that of the "ears" in the azimuth plot. The gaps in Figure 5.11 are due to angles of $\mathbf{d c o s z}$ for which a straight track does not cross 20 planes. For muons parallel with the z-axis, at trajectories near small dcosz, the number of planes a defocussed muon traverses increases because the magnetic field bends the particle in the z-direction, and this defocussing at this angle leads to a greater number of planes crossed and hence a greater acceptance.

Charge ratio data taken with a "reversed" field is shown as a function of azimuth in Figure 5.11, wherein the polarity of the current flowing through the coil was flipped, which is called Reverse field data. The biases are seen to have reversed, showing that this is indeed a bias inherent to the detector or reconstruction software and not an effect present in the cosmic muons. This will be discussed further in Chapter 6 . 


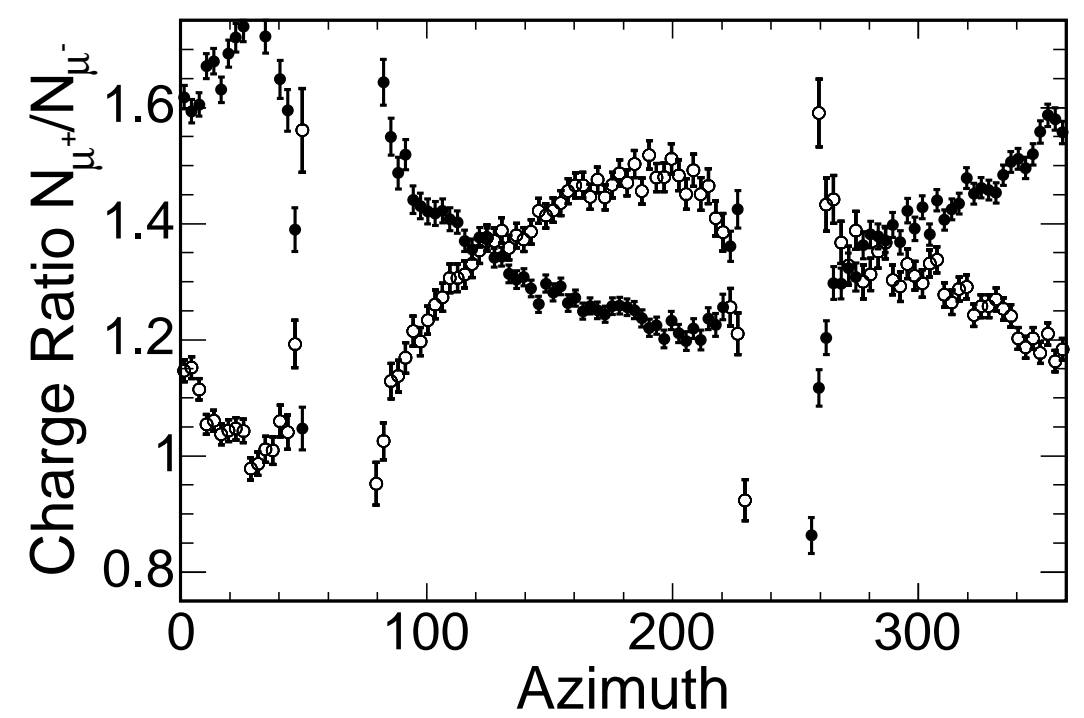

Figure 5.11: The charge ratio of muon tracks passing cuts as a function of azimuth, Forward field data is solid circles, Reverse field data is open circles.

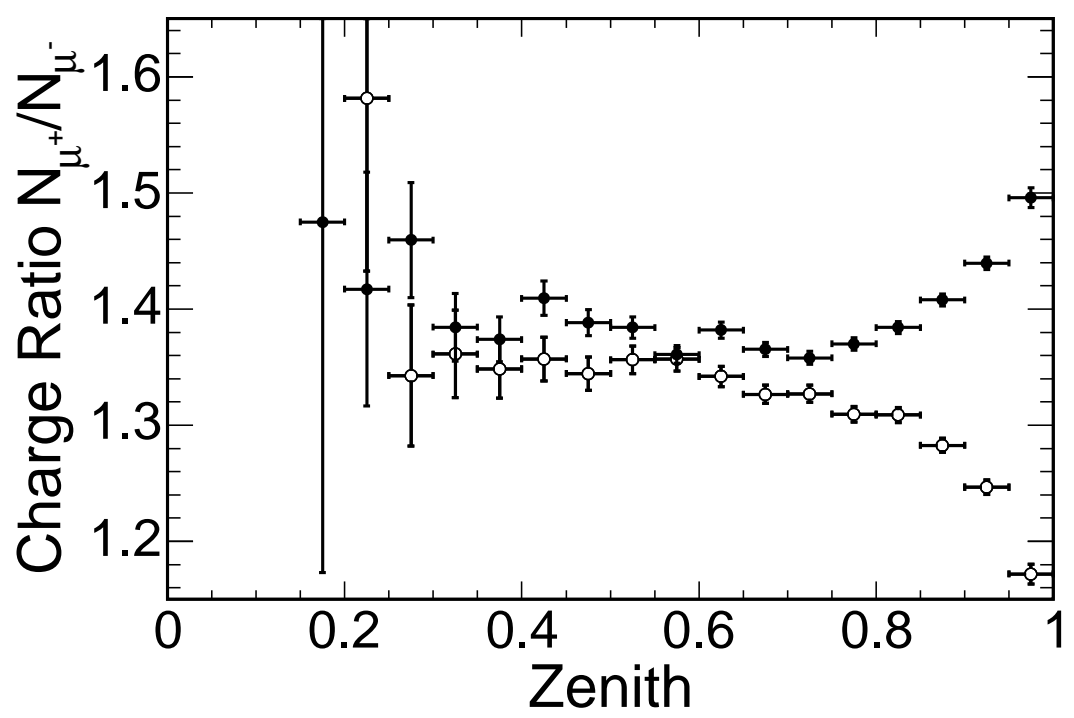

Figure 5.12: The charge ratio of muon tracks passing cuts as a function of zenith. 


\subsubsection{Detector Axis-Centered Coordinates}

The data that is plotted in Figures 5.11 and 5.12 show non-intuitive behavior due to the rapidly changing acceptance as a function of azimuth. By plotting instead the projection of a muon track to the plane containing the $\mathrm{x}$ - and $\mathrm{y}$-axes, insight may be gained. We call this " detector azimuth". Plotting the charge ratio as a function of detector azimuth in Figure 5.13 shows a clear East-West effect that remains regardless of cuts attempted. This effect has not been observed in the Monte Carlo. The shape of the effect also looks like a systematic error, especially considering the effect is very nearly the same in both supermodules. An explanation of this effect is not yet apparent.

\subsubsection{Data Purity Cut}

In Figure 6.5, the charge ratio is plotted versus the reconstruction tracking parameter $(q / p) / \sigma(q / p)$, which is a measure of the confidence of the charge assignment from the track fitter. Consider a sample of muons where the error of the curvature measurement exceeds the curvature measurement. This sample has poor confidence in their charge sign determination. It would then be expected that much of this sample would report a randomly chosen charge sign. This randomization would send the charge ratio to 1.0 and can be referred to as the randomization background, or the impurity of the charge determination. Conversely, by cutting out tracks that have a low-confidence in their charge determination, the purity of the charge determination is increased. As the level of confidence is increased, the purity increases but the efficiency decreases and hence the statistical error increases. In Figure 6.5, the charge ratio becomes nearly constant when $(q / p) / \sigma(q / p)$ is greater than 2.5 , or the percentage of the measurement that is error is less than $40 \%$. Increasing this cut 


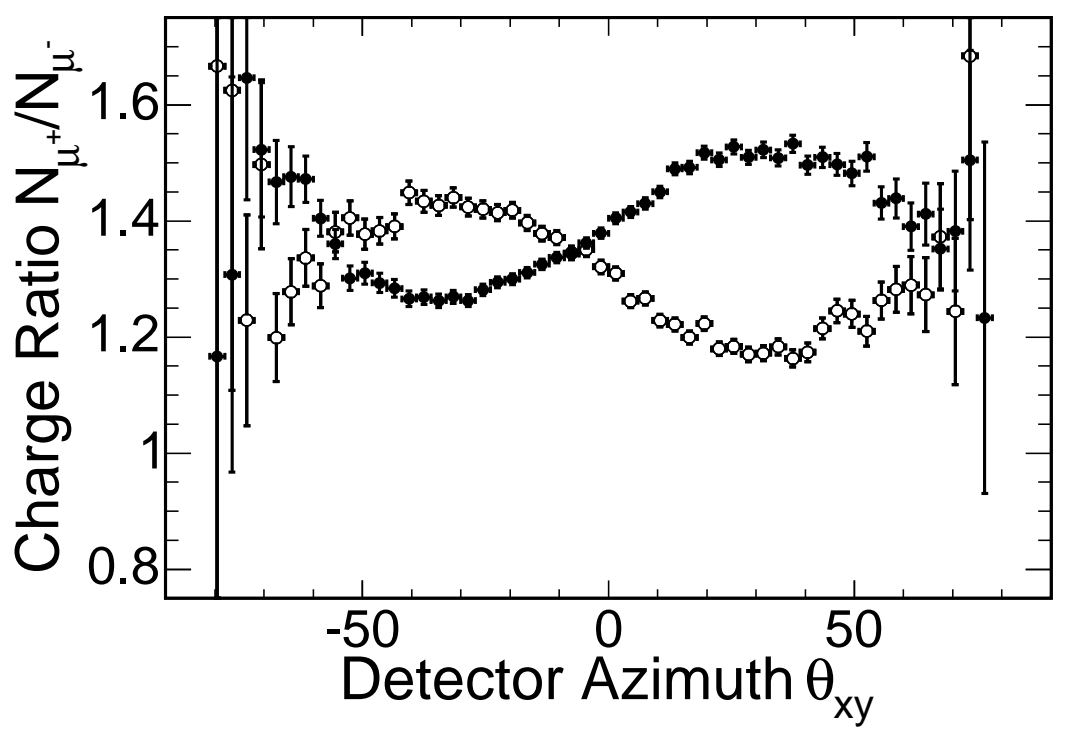

Figure 5.13: The charge ratio as a function of "detector azimuth" for Forward field data (solid) and Reverse field data (dashed). 
does not affect the charge ratio significantly, indicating that impurity from poorly determined momentum is removed.

\subsection{MINOS Cavern Rock Map}

Using the intensity determined from the Monte Carlo acceptance and the data, a "map" of the rock above the detector can be made in terms of slant depth and density of material that would be traversed by muons in a particular bin. The adjacent hall was constructed for the Soudan 2 experiment, which was approximately 60 meters from the MINOS location, and a rock map was produced using cosmic muons by S. Kasahara [3]. The Soudan 2 rock map is shown in Figure 5.14. It was produced by first determining the distance to surface from the detector using a Digital Elevation Map (DEM), provided by Geophysical Surveys. The elevation above the detector is shown in Figure 5.15. For this distance, the density was determined iteratively by first calculating the vertical intensity. Then, using the Crouch parameterization [17], the rock depth in standard rock was determined; density is this depth divided by the distance. The vertical intensity is dependent upon the rock density, providing a correction factor to the intensity used to find the depth in standard rock, so an iterative process was needed to find the actual density. The resulting density map is shown in Figure 5.16. The Soudan 2 detector has large acceptance gaps in particular in the zenith from $0-15^{\circ}$ and from azimuthal ranges of $80-100^{\circ}, 350-10^{\circ}, 170-190^{\circ}$ and $260-280^{\circ}$. These gaps in the resultant density map were filled with averages taken from nearest available bins. The original MINOS muon analysis [7] used the Soudan 2 rock map. Here I investigate corrections that may be needed.

The MINOS location is roughly 60 meters South and East of the Soudan site, so one bin in Azimuth, Zenith of the Soudan 2 rock map will point to a column of 
rock that is roughly 60 meters away and parallel to the correct column that was used in the Soudan 2 analysis. Density changes by about one percent when shifting by one or two bins, but the elevation above the detector shifts by as much as 80 meters when examining areas 60 meters apart on the surface, especially down the hill. Since the total distance to surface at high zenith angles is around 700 meters, the slant depth could change by $4 \%$ by using this incorrect elevation model. From an examination of Crouch parameterization, a change in slant depth of $4 \%$ anywhere in the range relevant to the Soudan lab would result in differences in intensity of as

much as $20 \%$. Initial surface muon energies would be modified by $4-5 \%$ as well. For these reasons, a new map using the Soudan 2 density and the corrected elevation model was created, which is shown in Figure 5.17.

\subsection{Examination of Muon Behavior in the Far Detector}

\subsubsection{High Energy, or Straight, Muons}

The MINOS detector's response to high energy muons can be modeled very simply by approximating the geometry as that of a uniform cylinder and all acceptances will be a function varying only as impact parameter "b" and dcosz. Such a study was undertaken to try to identify detector geometry effects separately from any reconstruction effects [31]. Approximately 4/5ths of the muons observed in MINOS will have energies above the resolution capability; ie, they are "straight", or hard, muons that will not curve significantly in the magnetic field. This is the sample that will be cut upon application of the QPID cut. A cylinder 30 meters long, with radius of 4 meters was used. The simulation addressed the following questions:

(1) for a given d dcosz and $\mathbf{b}$, what is the track length and number of planes that this muon is likely to see? 


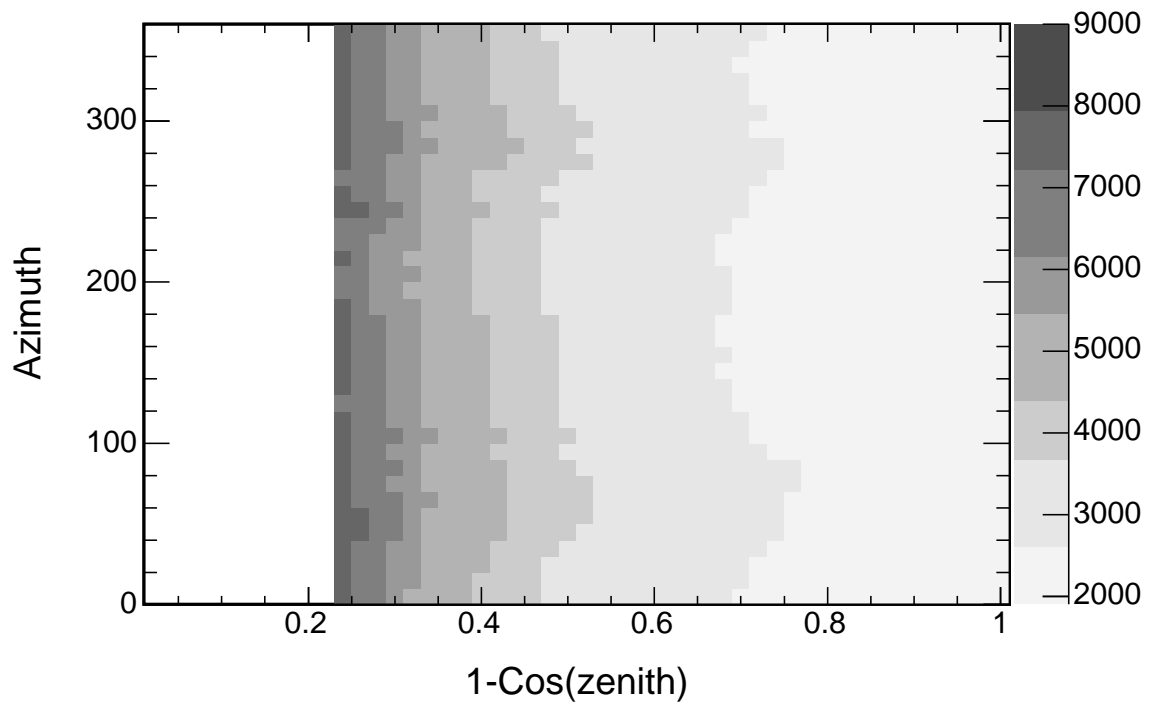

Figure 5.14: Map of rock traversed by muons in bins of azimuth and zenith, in M.W.E. as viewed from the center of the Hall floor underneath the Soudan 2 detector.

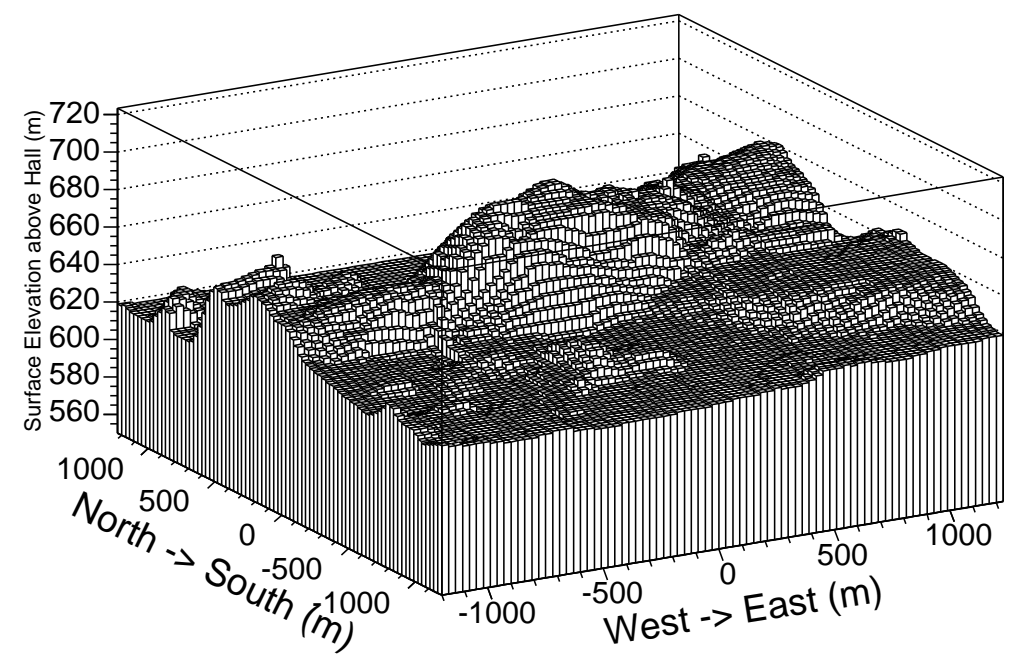

Figure 5.15: Surface elevation above the Soudan 2 detector, centered about the detector's coordinates. 


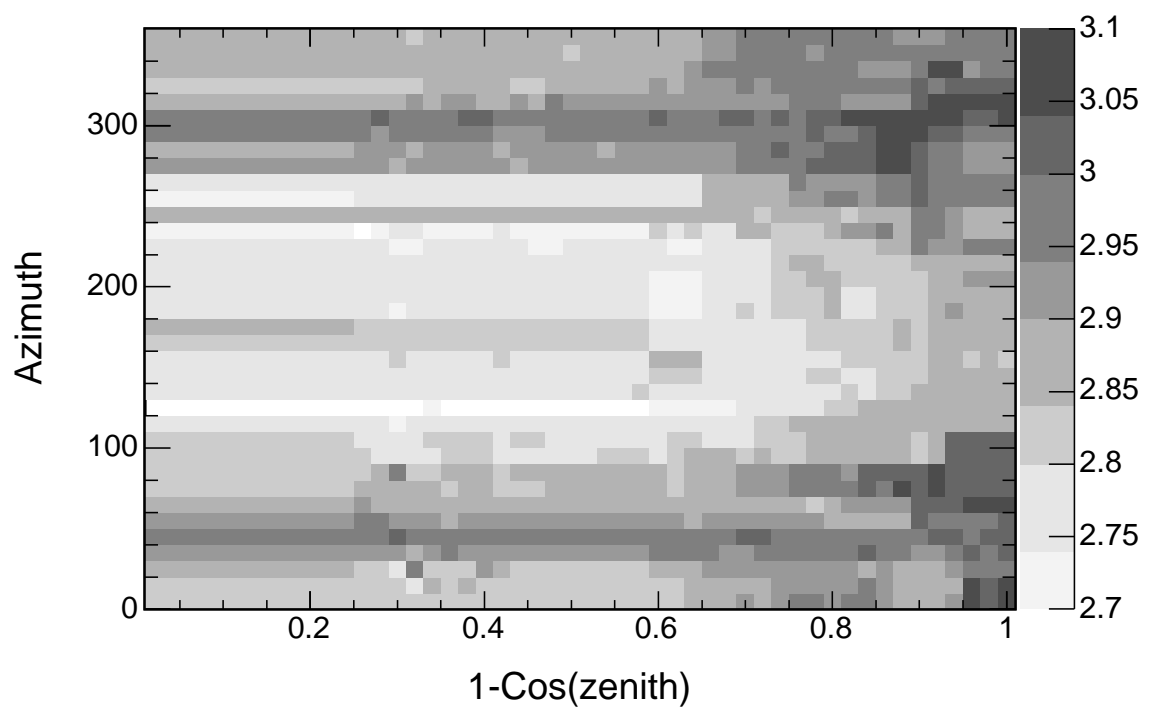

Figure 5.16: Map of rock density in bins of azimuth and zenith, in M.W.E. as viewed from the center of the Soudan 2 Hall floor.

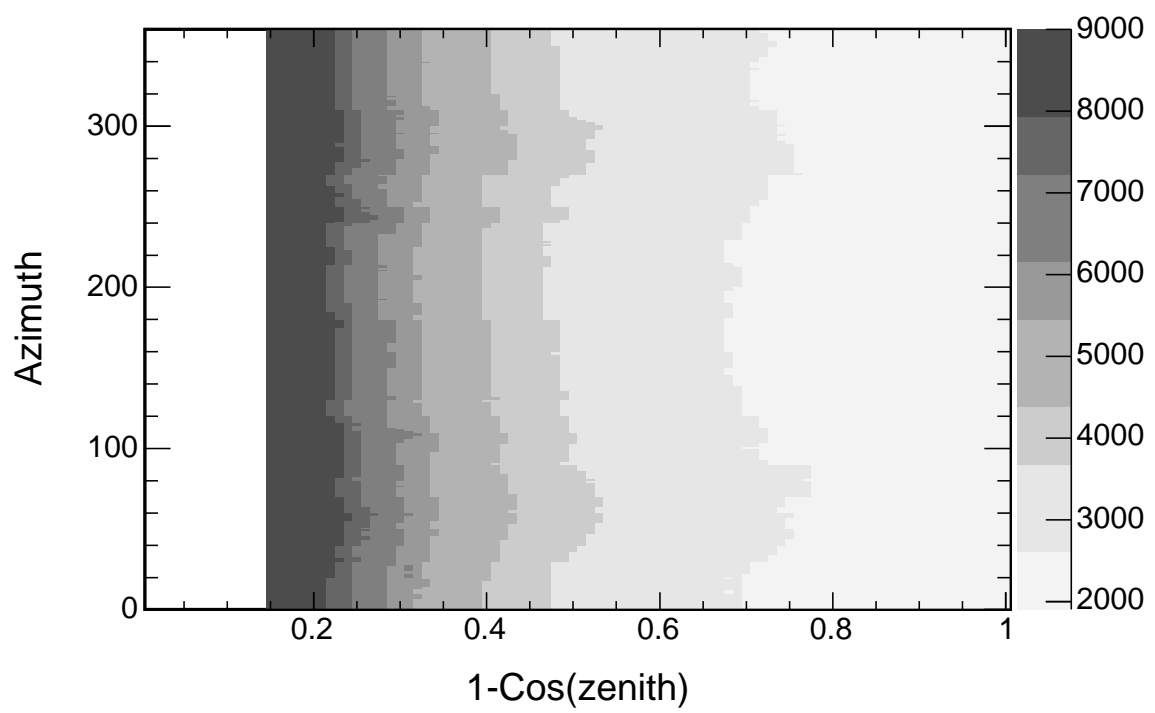

Figure 5.17: Map of rock traversed by muons in bins of azimuth and zenith, in M.W.E. as viewed from the center of the MINOS detector. 
(2) What proportion of muons at this dcosz will go through the outer ends of the two supermodules?

(3) What is the minimum momentum from range that would be observed?

Here simple equations are derived to answer these questions for straight tracks alone.

The perpendicular distance traversed is simply the chord of a 4 meter radius circle at impact parameter $\mathbf{b}$, or $2 \times \sqrt{4^{2}-b^{2}}$. The length of a straight track at this impact parameter is this distance divided by dsinz, which is the sine of the track's direction from the z-axis. Similarly, by taking the distance between planes as $5.94 \mathrm{~cm}$, the number of planes is then $\mathbf{d} \operatorname{cosz} \times L_{\text {track }}=33.67 \times \sqrt{4^{2}-b^{2}} \times d \cot z$, where $\mathbf{d} \operatorname{cotz}$ is the cotangent of the track's direction from the z-axis. Finally, the momentum range expected is the number of planes in the track times $0.04 \mathrm{GeV}$ divided by $\mathbf{d c o s z}$, or $p_{\text {range }}=$ planes $\times 0.04 G e V / d \cos z=1.347 G e V \times \sqrt{4^{2}-b^{2}} / d \sin z$.

The contamination of the data sample with tracks that either enter or exit either the South face or the North face for straight tracks can be approximated by dividing the length contaminated by end effects by the total length seen by muons. The amount of distance likely travelled in $\mathrm{z}$ by the simple approximations above is $z_{\text {track }}=$ planes $\times 0.0594 m=2 \times \sqrt{4^{2}-b^{2}} \times d$ cotz. The portion of sample contaminated by end effects is $2 \times z_{\text {track }}$, while total cross-sectional length of detector in $\mathrm{z}$ is $30 m+z_{\text {track }}$, so contamination fraction is $\frac{2 \times z_{\text {track }}}{30 m+z_{\text {track }}}$. This is more simply

written as $\frac{1}{0.5+\frac{d t a n z}{\sqrt{1+(b / 4)^{2}}}}$, which has the behavior of increasing slowly as dcosz is increased from 0 to 1 .

\subsubsection{Low Energy Muons, or Curvature in the Far Detector}

The charge of a particle tracked in the MINOS Far Detector is assigned by examining the curvature of that track. What follows is a calculation of the expected 
curvature with several simplifying assumptions. The results are given in Table 5.5 which show the strong dependence on dcosz and impact parameter.

\begin{tabular}{|l|c|c|c|}
\hline Momentum (GeV) & $|\mathbf{d c o s z}|$ & b $(\mathrm{m})$ & "sagitta" $(\mathrm{m})$ \\
\hline \hline 1.0 & 1.0 & any from 0 to 3.5 & 2 \\
1.0 & 0.0 & 0 & 0.667 \\
1.0 & 0.0 & 3 & 0.167 \\
10.0 & 1.0 & any from 0 to 3.5 & 0.2 \\
10.0 & 0.0 & 0 & 0.067 \\
10.0 & 0.0 & 3 & 0.017 \\
100.0 & 1.0 & any from 0 to 3.5 & 0.02 \\
100.0 & 0.0 & 0 & 0.007 \\
100.0 & 0.0 & 3 & 0.002 \\
\hline
\end{tabular}

Table 5.5: The expected curvature based on simplified detector and a track length of 8 meters: parallel to $\mathrm{z}$-axis is $\mathbf{d} \cos \mathbf{z}=1.0$, perpendicular is 0.0. For comparison, a strip's dimensions are 0.01 by 0.041 meters.

Assumptions: energy loss is negligible to first order for tracks of energies greater than $10 \mathrm{GeV}$, magnetic field is uniformly 1.5 Tesla and the particle's trajectory will have either an S-shape or a C-shape, depending on whether it passes a plane where the orientation of the field reverses.

\subsubsection{Two kinds/components of Tracks}

If a particle is going perpendicular to detector's z-axis, then its trajectory will be S-shaped since it experiences a field in one direction at first, then in the opposite direction once it crosses through the central axis. If a particle is parallel with z-axis, then its trajectory will most likely only be C-shaped in the simplest and by far most common case. Parallel with z-axis is simplest to treat, since it sees the full magnetic field vector at any place in the detector. Perpendicular to z-axis is a little more difficult, since the amount of magnetic field vector that is perpendicular to its trajectory at any point depends on impact parameter since field 
is cylindric. A particle with any angle in between the perpendicular and parallel will have components from each of these and can not be visualized effectively even to first order due to the complexity of the trajectories involved. So only the parallel and perpendicular (with impact parameter of 0) will be treated here.

\subsubsection{Calculation for parallel case}

Curvature of a charged particle in a magnetic field is given by Equation 5.4:

$$
R=\frac{p}{0.3 B} \approx 2 p
$$

Momentum $\mathbf{p}$ is in $\mathrm{GeV} / \mathrm{c}$, magnetic field $\mathbf{B}$ is in Tesla and $\mathbf{R}$ is in meters. All distances are in meters unless specified otherwise. For a track length $\beta$, the sagitta $\alpha$ is the largest distance of closest approach of this track to a straight line between the track's endpoints. Assuming no energy loss or multiple scattering, and that the field is uniform and always perpendicular to the track's velocity, then sagitta of a track with distance between endpoints given by $\mathbf{L}$ can be approximated in Equation 5.5:

$$
\alpha=R-R \sqrt{1-\frac{L^{2}}{4 R^{2}}}=2 p\left(1-\sqrt{1-\frac{L^{2}}{16 p^{2}}}\right)
$$

Using Equation 5.6 for series expansion of the square root term, and with $x=L^{2} /\left(16 p^{2}\right)$ which is always less than 1 (for a $1 \mathrm{GeV}$ muon, track length cannot be as much as 4 meters) to get the result of Equation 5.7:

$$
\begin{gathered}
\sqrt{1-x}=1-\frac{x}{2}-\frac{x^{2}}{8}-\frac{x^{3}}{16}-\ldots \\
\alpha \approx \frac{L^{2}}{8 R} \approx \frac{L^{2}}{16 p}
\end{gathered}
$$


This can also be parameterized in terms of number of additional strips crossed away from straight line (parallel to z-axis, strip width is $4.1 \mathrm{~cm}$ ) in Equation 5.8:

$$
N_{s} \approx \frac{L^{2}}{0.64 p}
$$

This calculation assumes the field is constant in magnitude everywhere. Between the steel sheets, the field is negligibly small, so the field only fills about $43 \%$ of the space. This can be approximated as reducing the field overall so that $\mathrm{R} \approx 4$ p. This propagates through to give Equation 5.9:

$$
N_{s} \approx \frac{L^{2}}{1.28 p}
$$

With L as 8 meters, $N_{s} \approx 50 / p$. For a muon with $10 \mathrm{GeV}$ energy and 8 meters between endpoints ( $\mathrm{L} \approx \beta$ to first order and most of the time to second order), $N_{s} \approx 5$. For $50 \mathrm{GeV}, N_{s} \approx 1$.

\subsubsection{For perpendicular case (impact parameter 0)}

In this case, the muon will be curved in the z-direction, rather than the radial direction. And its curvature will flip when the muon passes the midpoint of the detector. So this decreases the distance between endpoints that sagitta can be measured from by factor of 2. Additionally, the distance between strips is now increased to $5.94 \mathrm{~cm}$ when viewed this way, since this is the spacing between planes.

So $N_{s} \approx L^{2} /(1.9 p)$, with $\mathrm{L}$ as the distance between endpoints of one half of the S-shape.

With L equal to 4 meters, $N_{s} \approx 8.4 / p$. For $10 \mathrm{GeV}, N_{s}$ is close to 1 . Therefore, the momentum/QPID capabilities are reduced by a factor of 5 when looking at muons that traverse perpendicular to the z-axis versus muons that traverse parallel 
to z-axis of the detector.

\subsubsection{Curvature at non-zero Impact Parameter}

Impact parameter only affects the curvature of the muon by the muon's perpendicular component of velocity. For muons parallel to the z-axis, impact parameter doesn't matter as much. But for the perpendicular component, as impact parameter $b$ increases from zero, the initial magnetic field component perpendicular

to the incoming track goes as $\sin \left(\cos ^{-1} \frac{b}{4}\right)$, where $b$ is in meters. Now as the muon traverses the detector, the amount of magnetic field perpendicular to its travel now goes as $\sin \left(\cos ^{-1} \frac{b}{r}\right)$, where $\mathrm{r}$ is the radius from the center of the detector, which is shown in Figure 5.18.

This has the result of modifying the relation between momentum and radius of curvature like $\mathrm{R} \approx 2 p / \sin \left(\cos ^{-1}(b / 4)\right)$. As impact parameter increases beyond 3 meters, $10 \mathrm{GeV}$ becomes the maximum detectable momentum for tracks with dcosz near 0 .

\subsubsection{Curvature in Earth's Magnetic Field}

Referring to equation 1.1 with $\alpha \approx \frac{L^{2}}{8 R}$, and for the angular deviation using $\sin \theta \approx \theta=\frac{\alpha}{60,000 \text { meters }}$, the Earth's magnetic field of 0.5 Gauss gives $R \approx$ $66,000 \frac{m}{G e V} p$, with $\mathrm{p}$ in $\mathrm{GeV}$. The minimal average energy of a muon travelling to the detector is $250 \mathrm{GeV}$, and the maximal average energy that can be charge separated is about $3 \mathrm{TeV}$. This gives a minimal deviation of 0.04 milliradians and a maximal deviation of 0.55 milliradians. 


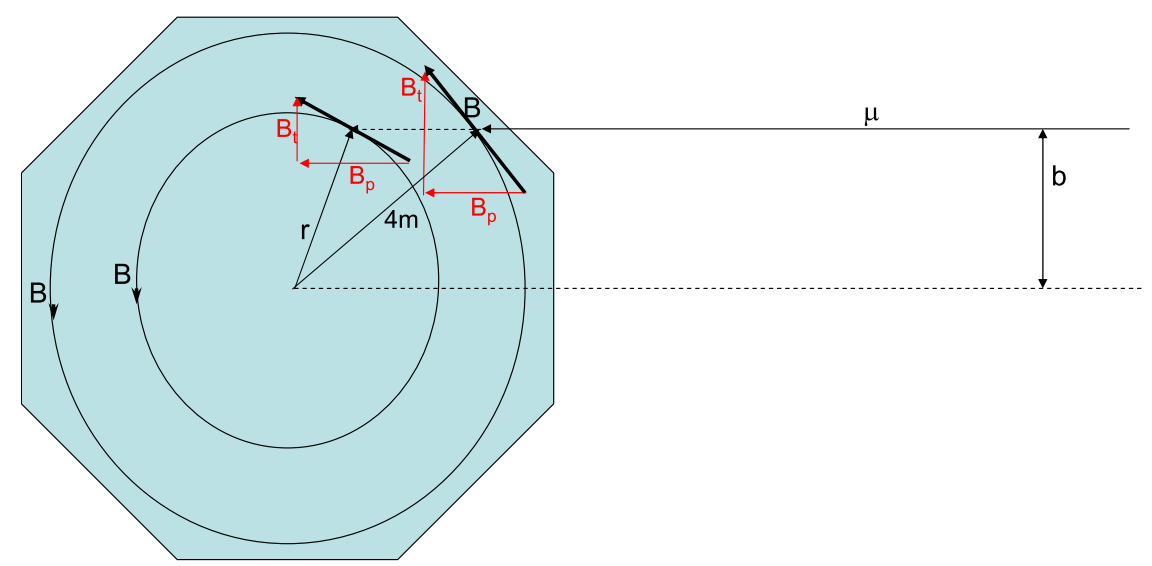

Figure 5.18: The Magnetic Field seen by a muon's perpendicular component (in the xy-plane) as it traverses the detector for impact parameter $\mathbf{b}$. 


\section{Chapter 6}

\section{Charge Determination in MINOS}

\subsection{Summary of the Problem}

Within a year of magnetized data taking in 2002, unexpected asymmetries in the charge ratio had been reported in many distributions, particularly in the azimuth as defined in Chapter 4. Several plots from Brian Rebel's analysis with Forward field data can be seen in Figures 6.1-6.3 [7]. This problem was originally observed as primarily a North/South effect with some East/West structure, neither of which were noticed in the Monte Carlo. The charge ratio is plotted as a function of azimuth for single tracks that traversed at least 20 planes and 2 meters of track length in Figure 6.4. Previous work on this problem is summarized in Brian Rebel's thesis [7]. The azimuth of a muon is the direction that the muon comes from, determined from its vertex direction cosines. So an azimuth near $0^{\circ}$ means the muon is coming from the North, and so it is "South-going".

In January of 2004, the charge ratio seen was increased by using a track charge identification quality cut, or the "QPID cut". Plotting the charge ratio versus error

of the curvature determination divided by the curvature, or $\frac{\sigma(q / p)}{q / p}$, the charge ratio level moves further away from 1.0 and levels out once $\sigma(q / p)$ is less than $40 \%$ of $\mathrm{q} / \mathrm{p}$.

This cut removes all events where the track fitter returned a poorly determined 

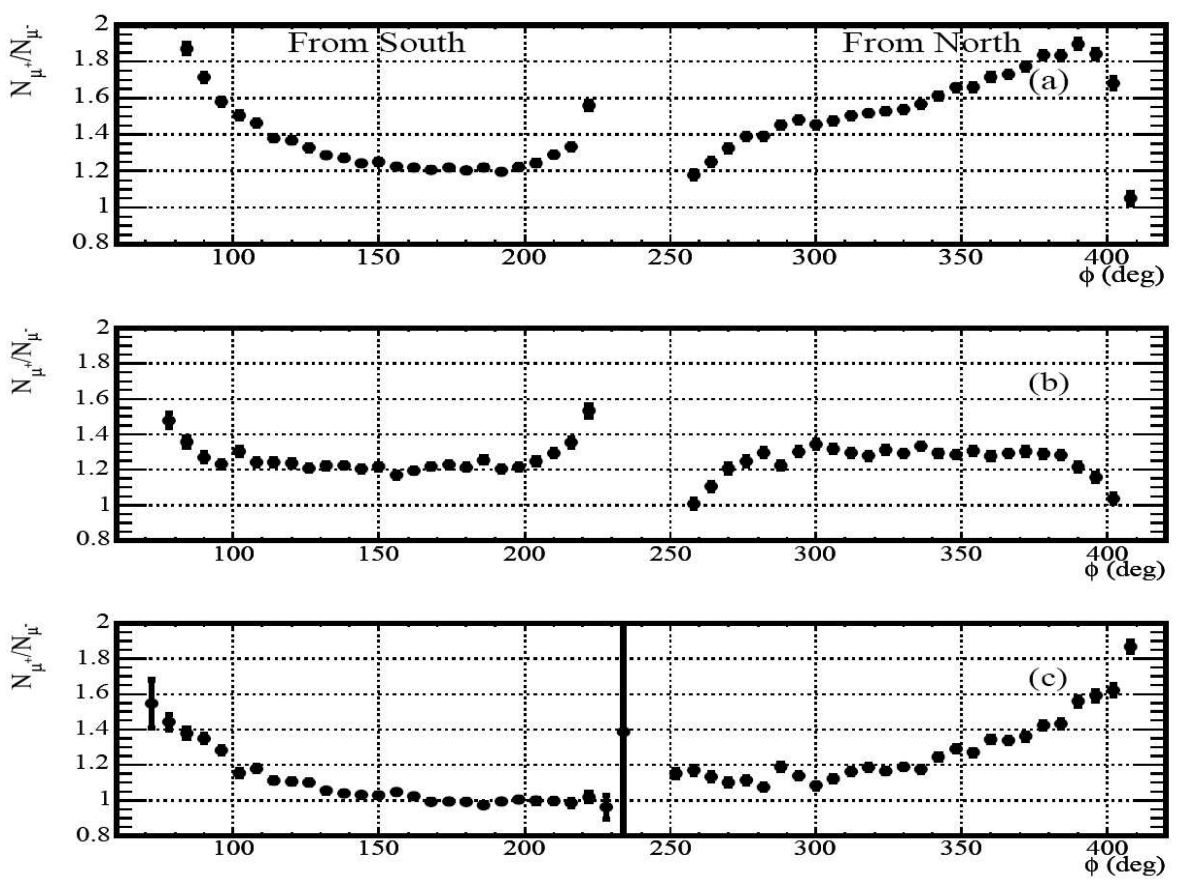

Figure 6.1: Charge Ratio as a function of the azimuth in MINOS Far Detector, (a) is Forward field data, (b) is Monte Carlo and (c) is Data divided by MC, from [7]. 

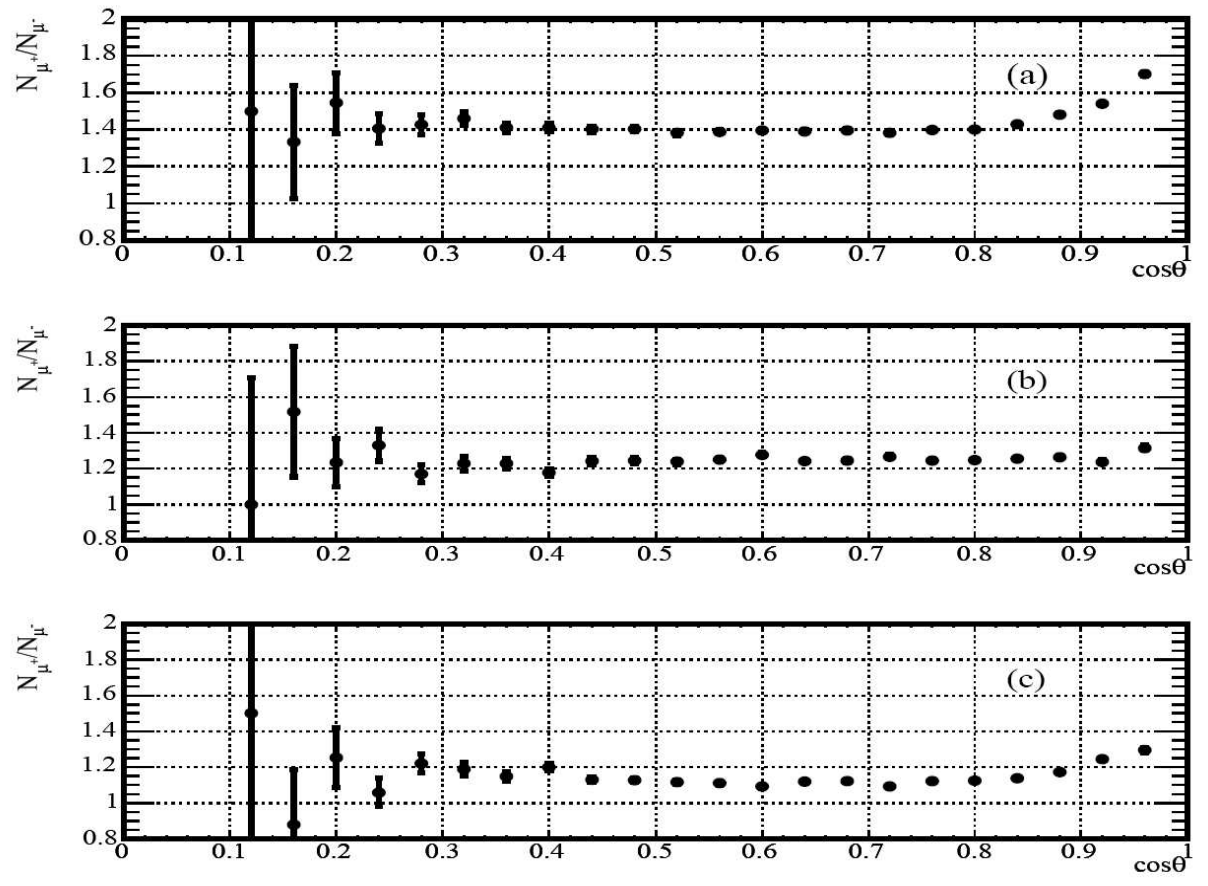

Figure 6.2: Charge Ratio as a function of the zenith in MINOS Far Detector, (a) is Forward field data, (b) is Monte Carlo and (c) is Data divided by MC, from [7]. 

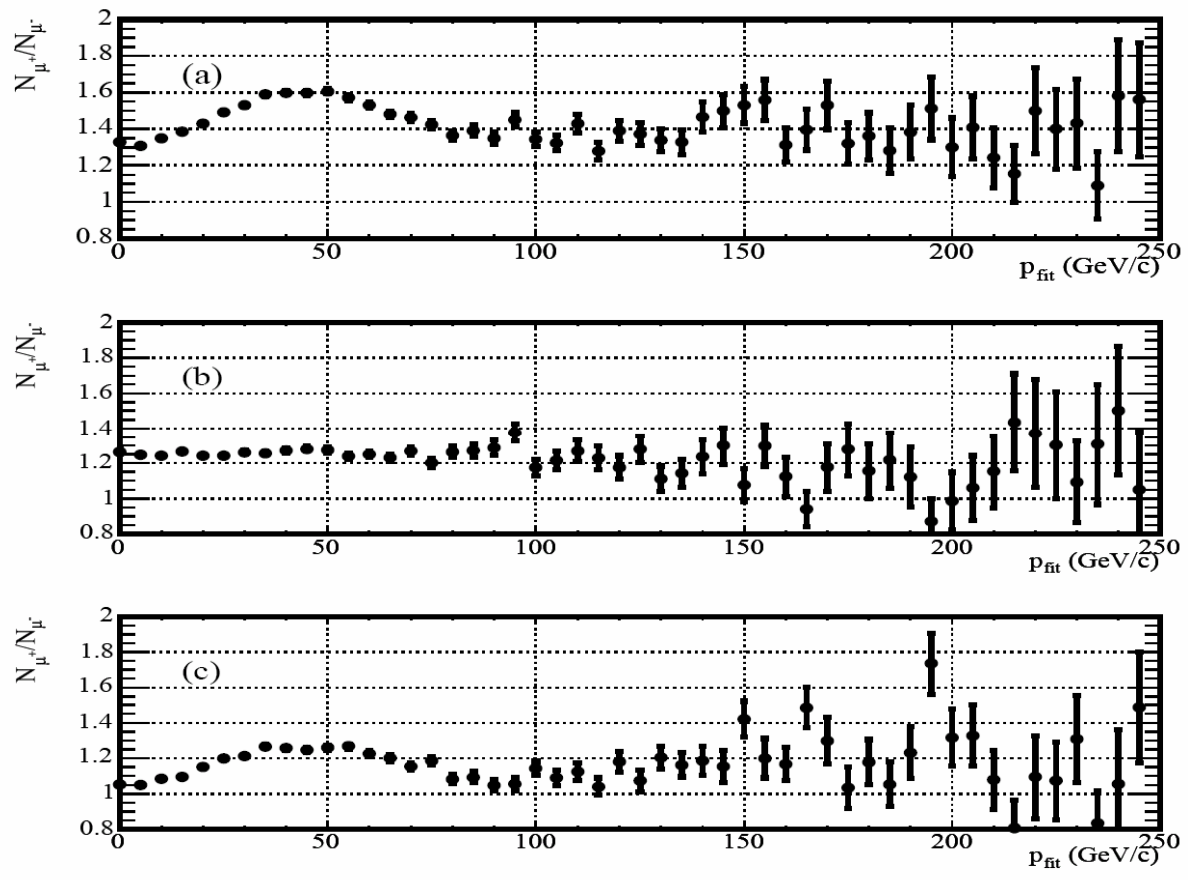

Figure 6.3: Charge Ratio as a function of the reconstructed momentum in MINOS Far Detector, (a) is Forward field data, (b) is Monte Carlo and (c) is Data divided by $\mathrm{MC}$, from [7].

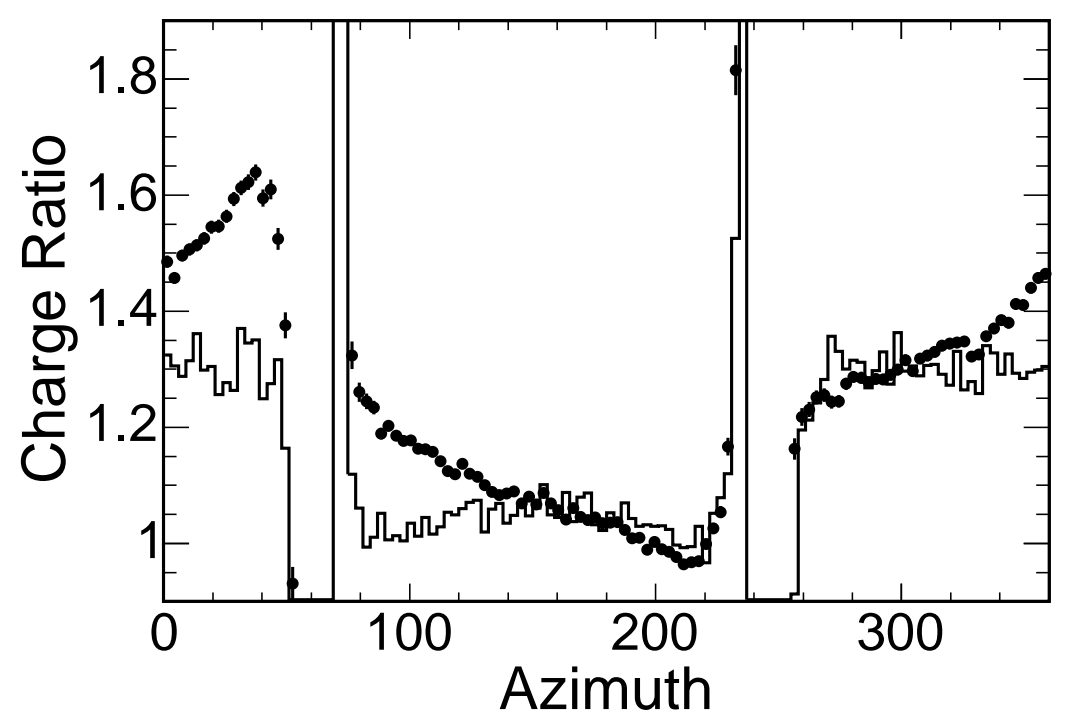

Figure 6.4: Charge Ratio versus azimuth, no QPID cut, MC is a solid line, data is filled circles. 


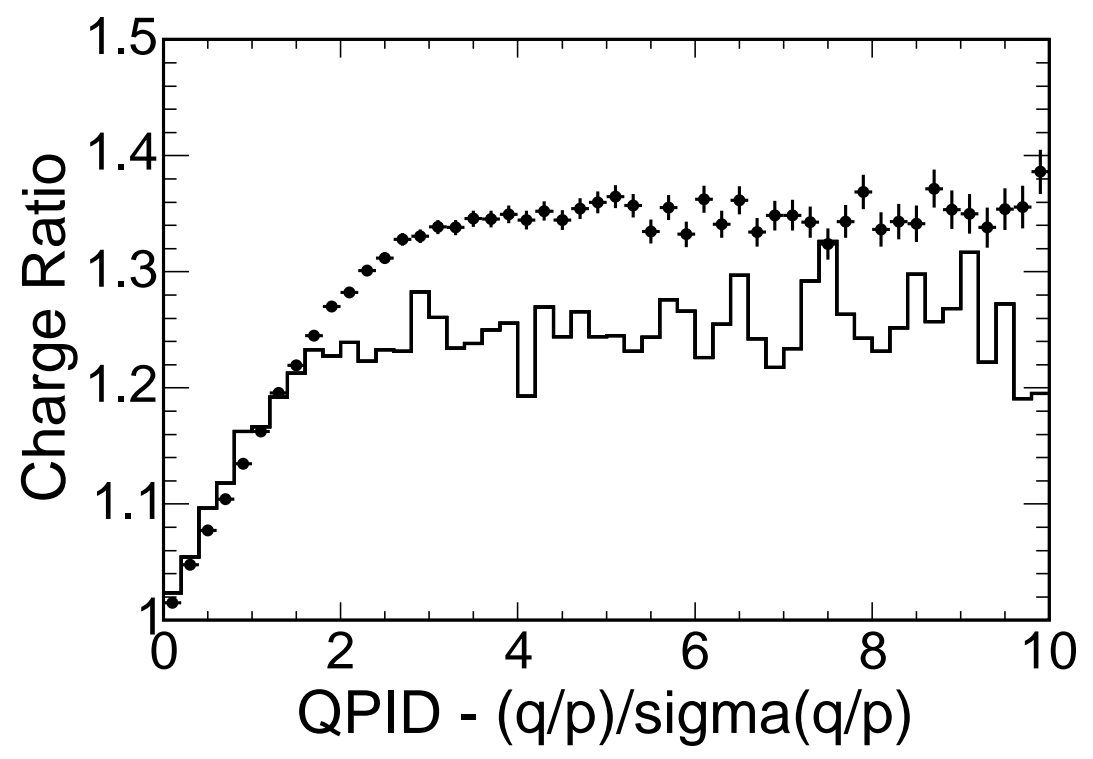

Figure 6.5: The Charge Ratio versus the "QPID cut": $(q / p) / \sigma(q / p)$. The Data is points with error bars while the MC is the solid line. Note: MC has an input charge ratio of 1.25 . 
charge. After making this cut however, the qualitative shape of the asymmetries remained, but the average charge ratio moved further away from 1.0. Poor QPID is associated with randomly assigned charge, and so this mix of reconstructed charges will be 50-50 positive/negative, pushing the charge ratio towards 1.0, so cuts are needed to remove this impurity from randomization (background) and get a true charge ratio value. This cut was determined empirically from the plot of the data charge ratio versus the QPID.

In Figure 6.6, the charge ratio is plotted as a function of azimuth both before and after the QPID cut, and this effect of pushing the charge ratio away from 1.0 is seen.

The two fundamental problems with the data sample are the bias and the background. Bias was discussed above, and based on the charge ratio plots in forward and Reverse field, is at most 5\%. Bias is the behavior seen in the detector but not in the Monte Carlo. Background is the contribution in each sample from random QPID, which was discussed above with the empirical QPID cut. This is seen in the Monte Carlo as well, although the QPID at which the charge ratio levels off is higher in data than in MC. This indicates the resolution of the MC is better than that of the real detector for whatever reason, so the cut must be based on data. Additionally, QPID seems to depend on dcosz, which is shown in Figure 6.7 with the result that the QPID cut as used in this analysis reflects this dependence on dcosz.

In mid-2004, the magnetic field was reversed. The qualitative asymmetries in the charge ratio reversed, proving that the problem was associated with the detector. A positive track with a specified azimuth, zenith, momentum, and vertex position will proceed through exactly the same space in the detector with the field in forward polarity as a negative track in the reversed polarity magnetic field, assuming 


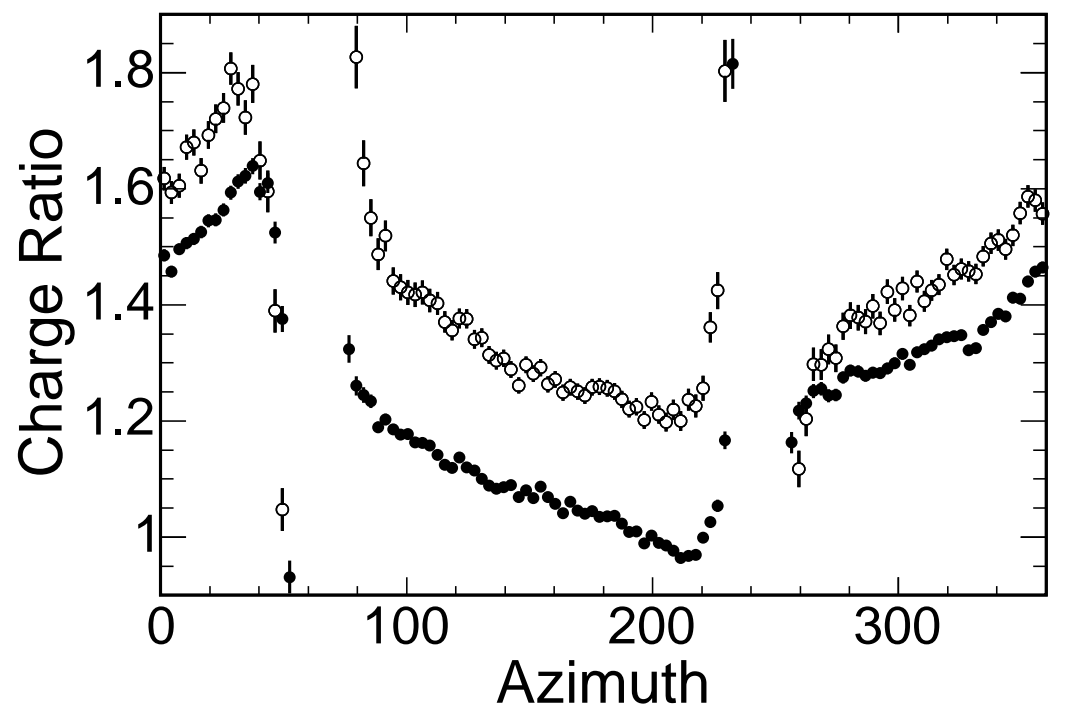

Figure 6.6: The charge ratio versus azimuth, Forward field Data before QPID cut is in filled circles, same data after the QPID cut is displayed in open circles. 

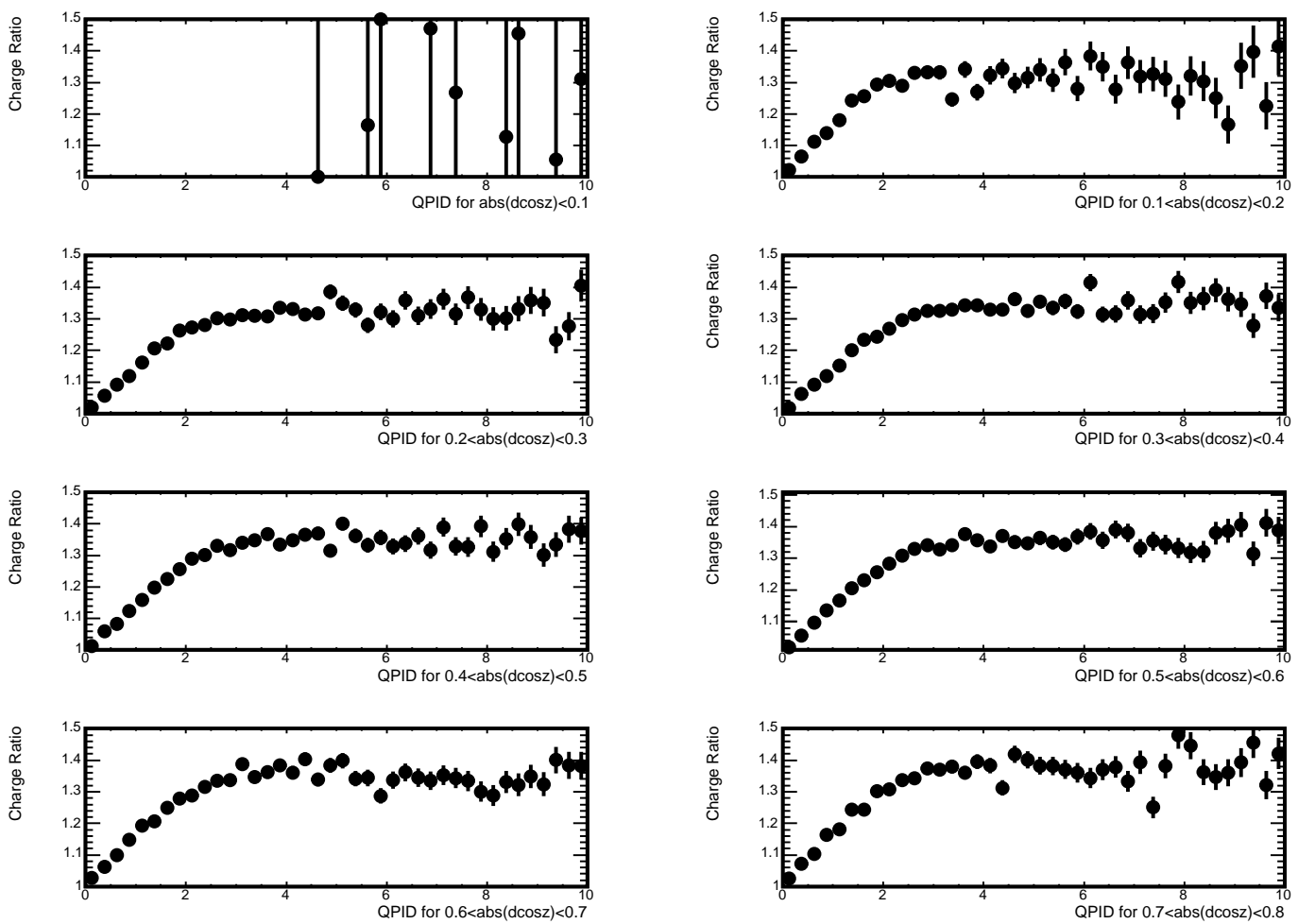

Figure 6.7: Summed charge ratio versus QPID for a range of $|\mathbf{d} \operatorname{cosz}|$. The point at which charge ratio levels off indicating background is removed, is a function of dcosz. 


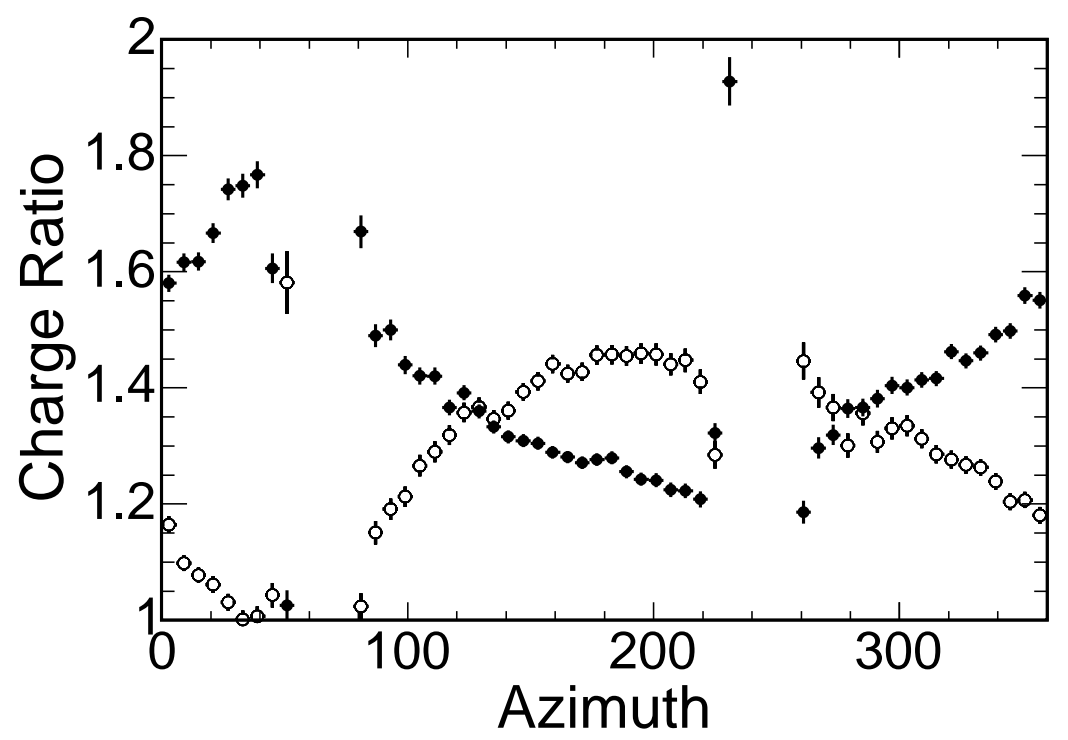

Figure 6.8: Two charge ratio data sets versus azimuth, Forward is in dark circles, Reverse is in open circles. 
that the field reverses exactly in every spot. This is not a trivial assumption and will be examined in Chapter 7 in more detail. If this assumption is correct, then by summing the positive muons in the forward field with the positive muons in the reverse field, doing the same with the negative muons and finally taking a ratio, we have a result that is free of any QPID bias present in the detector, so long as each muon sample is normalized by the livetime (integrated data acquisition time) of each field configuration.

The two "Alignment Data Sets" are each two week magnet off data samples for each SuperModule (SM) taken for alignment purposes. The data was taken before the magnet in the respective SM was energized for the first time, so the muons should experience no net curvature. The sets were reconstructed as though the field were on in the Forward field configuration, and the qualitative asymmetries showed up here as well. This implies very strongly that this East/West bias was not caused by the magnetic field but was somehow otherwise inherent to the detector. This is shown as a fake charge ratio since these muons are not actually positive or negative but are merely reconstructed as such in Figures 6.10 and 6.11; note the ratios are centered about 1.0 implying overall random charges. The reconstruction of this data took place after the alignment procedure was run using this same data and residuals resulting from straight line fits [30]. Correlated errors in the alignment procedure, or a bug in the geometry were viewed as possible suspects.

The field off data was examined for systematic biases in the straightness of the tracks. Multiple scattering does introduce random curvature, but it is uncorrelated, and so it should average out. The sagitta of a track is taken as the largest deviation from a straight line fit to the track. The positions of these sagitta, or where they occured along a muon track, were plotted in U,Z and V,Z space in Figure 6.12.

Systematic biases can be observed in the plot which imply that the response of 


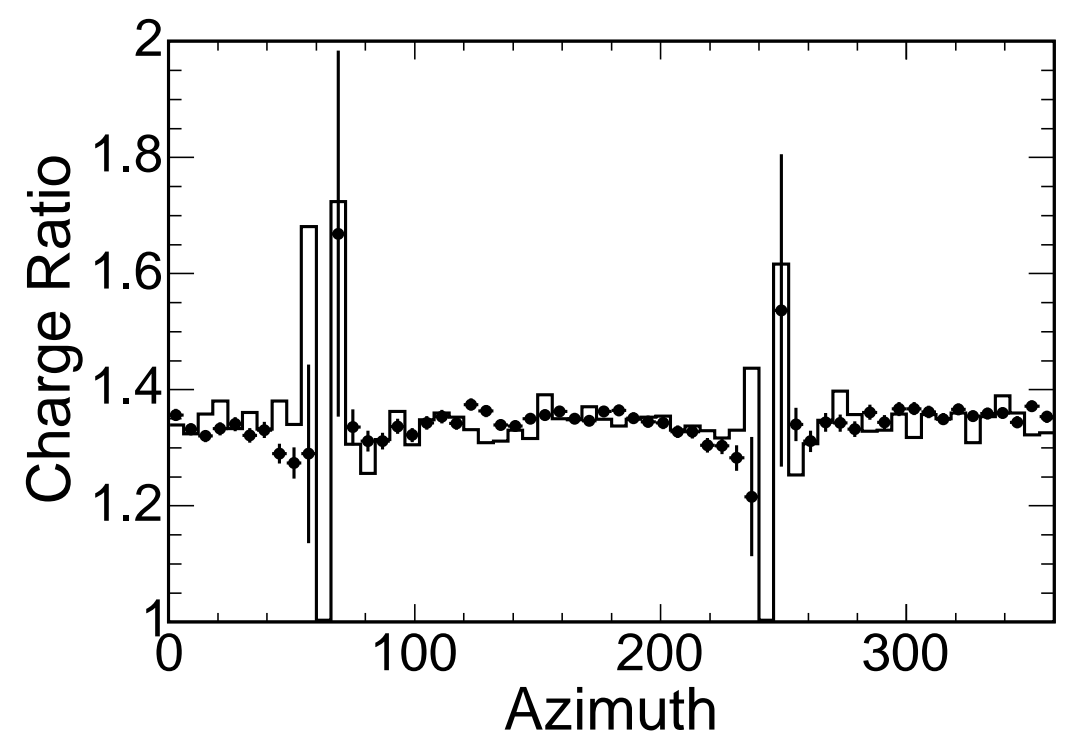

Figure 6.9: Normalized and summed Forward and Reverse data sets, data is in dark circles, MC is the solid line and has been scaled to have the same charge ratio as the data (1.347).

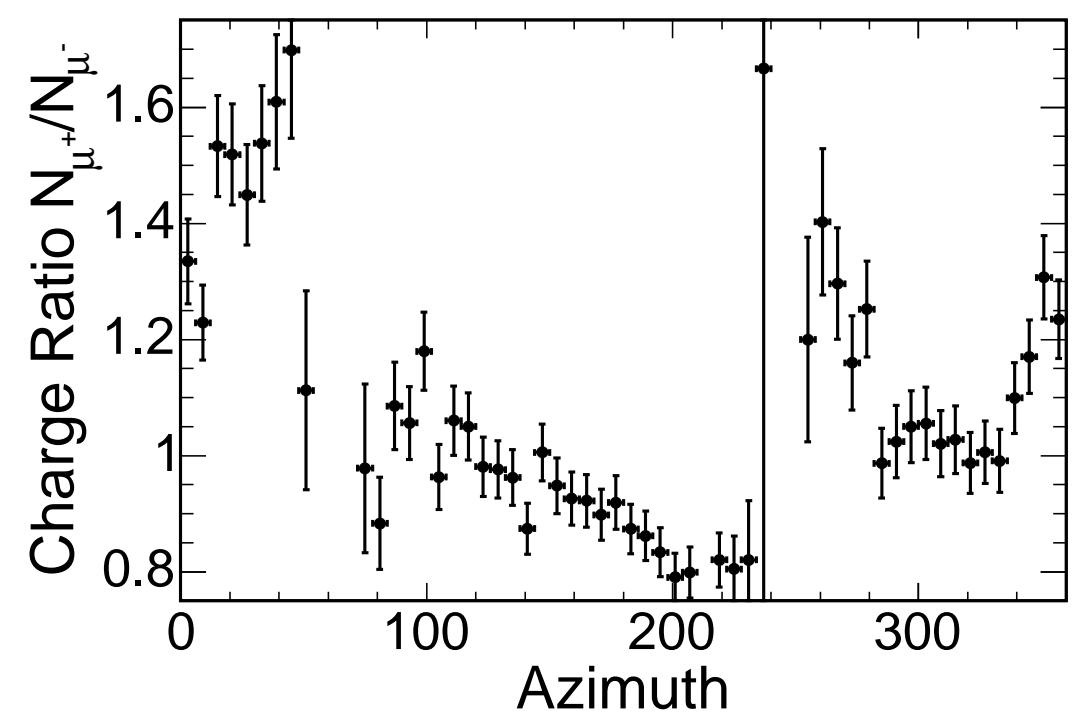

Figure 6.10: The two-week SM1 "Alignment Data Set's" charge ratio versus azimuth. 


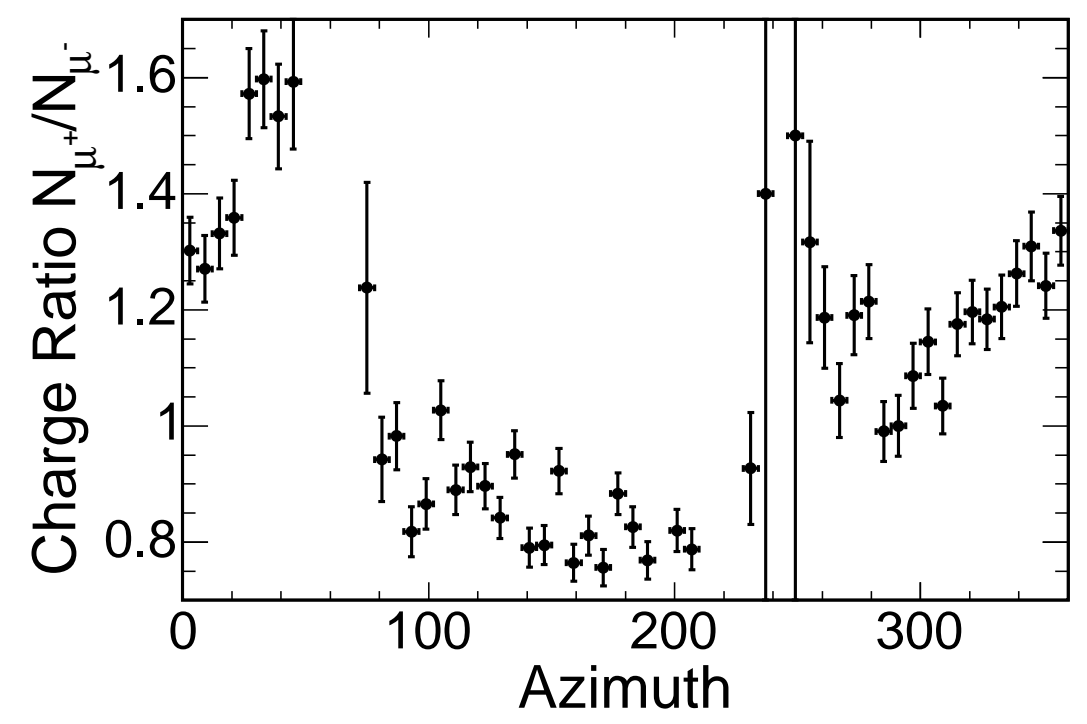

Figure 6.11: The two-week SM2 "Alignment Data Set's" charge ratio versus azimuth.
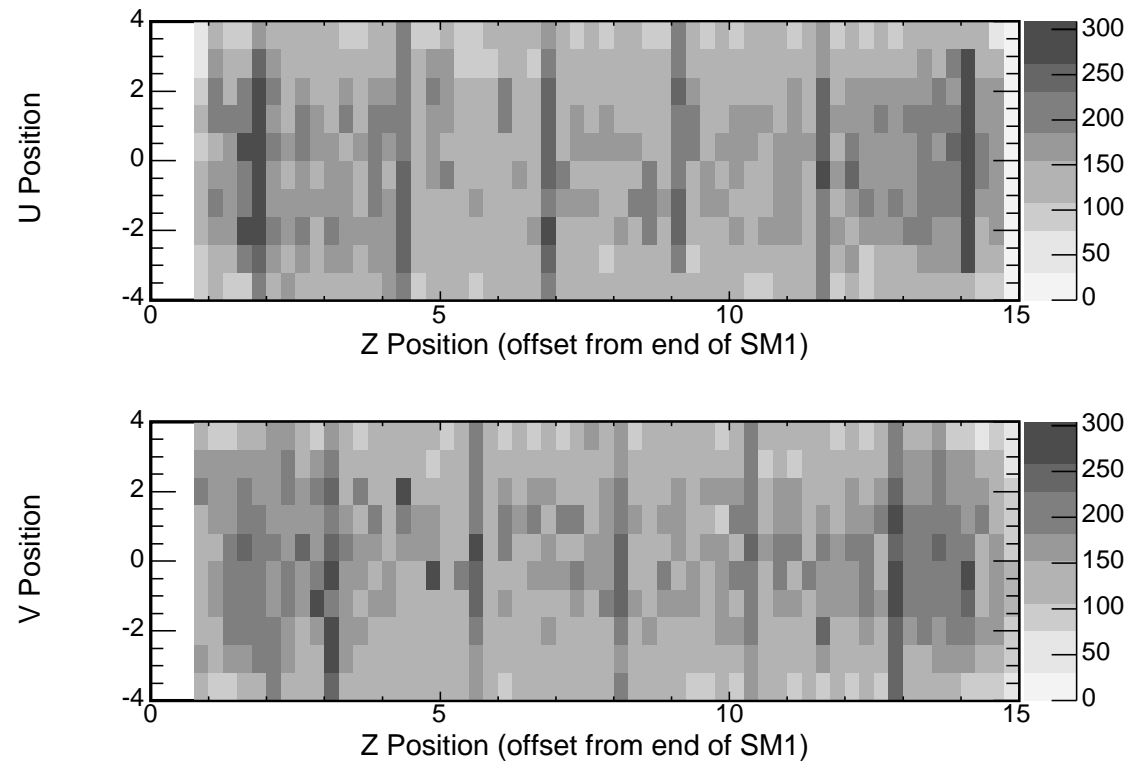

Figure 6.12: Sagitta positions in the detector for SuperModule 2 field off data. 
the detector to curvature of tracks is not uniform throughout the detector. Maximal curvature seems to preferentially occur in bands at periodic points along the z-axis. Again, alignment is viewed as the major possible culprit.

This chapter will explain the charge ratio data and the work done to eliminate this effect.

\subsection{Magnetic Field}

The magnetic field was viewed as a possible problem in the reconstruction, because it was not initially possible to quantify our confidence in the field map used. It was also well known that several effects in our coil setup were not being modeled, such as the return leg of the coil underneath the detector, the correct chemistry and $\mathrm{BH}$ hysteresis data for the steel, the outer 20 planes at each of the $4 \mathrm{SM}$ ends having sequentially lower and lower contained field per plane, and also magnetic field outside the steel. These effects were all ignored, and an incorrect current was applied in the ANSYS calculations. The addition of these effects to the field model has changed the field maps by as much as $10 \%$, but the charge ratio asymmetries have not changed significantly from using these improved maps. Previous work [7]

indicated the charge ratio problem is relatively insensitive to the field. One study scaled the field maps by $10 \%$ uniformly greater or less than nominal and the other scaled $10 \%$ of the planes, chosen at random, by $5 \%$ greater or less than nominal. These changes did not affect the ratio asymmetries.

\subsubsection{An Argument Against the Field Being the Problem}

The result of adding these effects to the model only significantly altered the magnitude of the field, and to a smaller degree the vector direction of the field at places on the field map were altered. Never did the addition of these effects result in 
the flipping of a field vector anywhere on a field map. For all the points on a track through the MINOS detector, only inverting the field vector could result in changing the sign of charge identification. Changing the field magnitude would result in a change in the momentum magnitude determination, but not the sign of the charge. However, effects could be present in our steel to change the overall shape of the field, which led to a study using a toy Monte Carlo developed initially for estimating the acceptance of the detector.

\subsubsection{Toy Monte Carlo}

The toy Monte Carlo projected muons on a magnetized cylinder the size of the Far Detector. The cylinder was homogenous (no planes or strips were simulated) and an average energy loss per step was calculated and muons were "tracked" through in steps. At the end of the process, the question "were the equivalent of 20 planes passed through" was asked, which is more simply put as "was the distance traversed

in Z more than 1.2 meters"? So this simulation only looked at whether or not a muon would traverse 20 planes, and this ended up being very useful, because this toy Monte Carlo reproduced many features of the full Monte Carlo, with very small turn-around times. This calculation only considered effects of the geometry and magnetic field, but not the reconstruction software. A full discussion of the results of this study can be seen in [31].

\subsubsection{Modified Field Map}

It was proposed that the magnetic field could be the cause of the asymmetry, in that it could be modeled incorrectly. To determine the effect of a magnetic field that was not mostly cylindrically symmetric, a "squished" field was put into the toy Monte Carlo to see if the earlier East/West effect could be reproduced. The 
field that was used is shown in Figure 6.14 that modified a circular bfield that goes as $1 / r^{1.1}$ by squishing the $\mathrm{u}$-axis (45 degrees to $\mathrm{x}$ and $\mathrm{y}$ axes). The result of the study is shown in Figure 6.15 and it was determined that even with a high degree of modification, the charge ratio as a function of angle about the xy plane did not show the effect seen in the data.

\subsection{Alignment}

Referring to the end of Chapter 4 on coordinate systems, recall that the scintillator positions are not precisely aligned due to construction deviations allowed

for by the set tolerances. Knowledge of the actual position of a scintillator strip is required for accurate determination of the position of a hit and the accurate and bias-free reconstruction of a track going through that strip.

\subsubsection{Longitudinal, or Z-axis Alignment}

The system discussed at the end of Chapter 4 is the "ideal" local coordinate system, and deviation from this ideal is the "actual" local coordinate system. Any deviations from the ideal are due to construction or shifting of the support beams. The entire hall itself has in fact shifted during construction due to the extra weight being placed in the middle of the hall after two years of being empty. Of fundamental importance to the discussion is the requirement of the actual coordinate system to be interchangeable with the coil hole of the Far detector. Regardless of construction deviations, the coil must slide down a tube with very little imperfection (compared with any other deviations as will be seen shortly) to be at the center of the magnetic field. Ultimately, the scintillator and steel planes are centered about the coil tube, and this must be taken as the actual coordinate system, since for all purposes, scintillator strips line up around the coil hole near $\mathrm{x}=0$ and $\mathrm{y}=0$. 


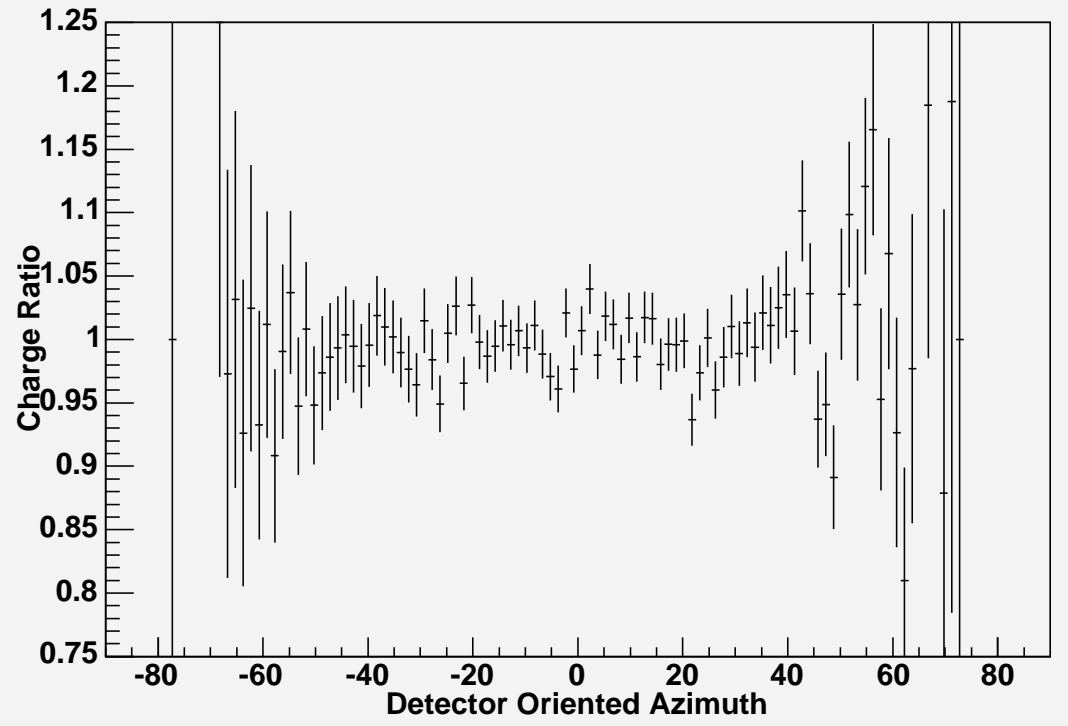

Figure 6.13: The charge ratio as a function of detector azimuth for the Toy Monte Carlo. 


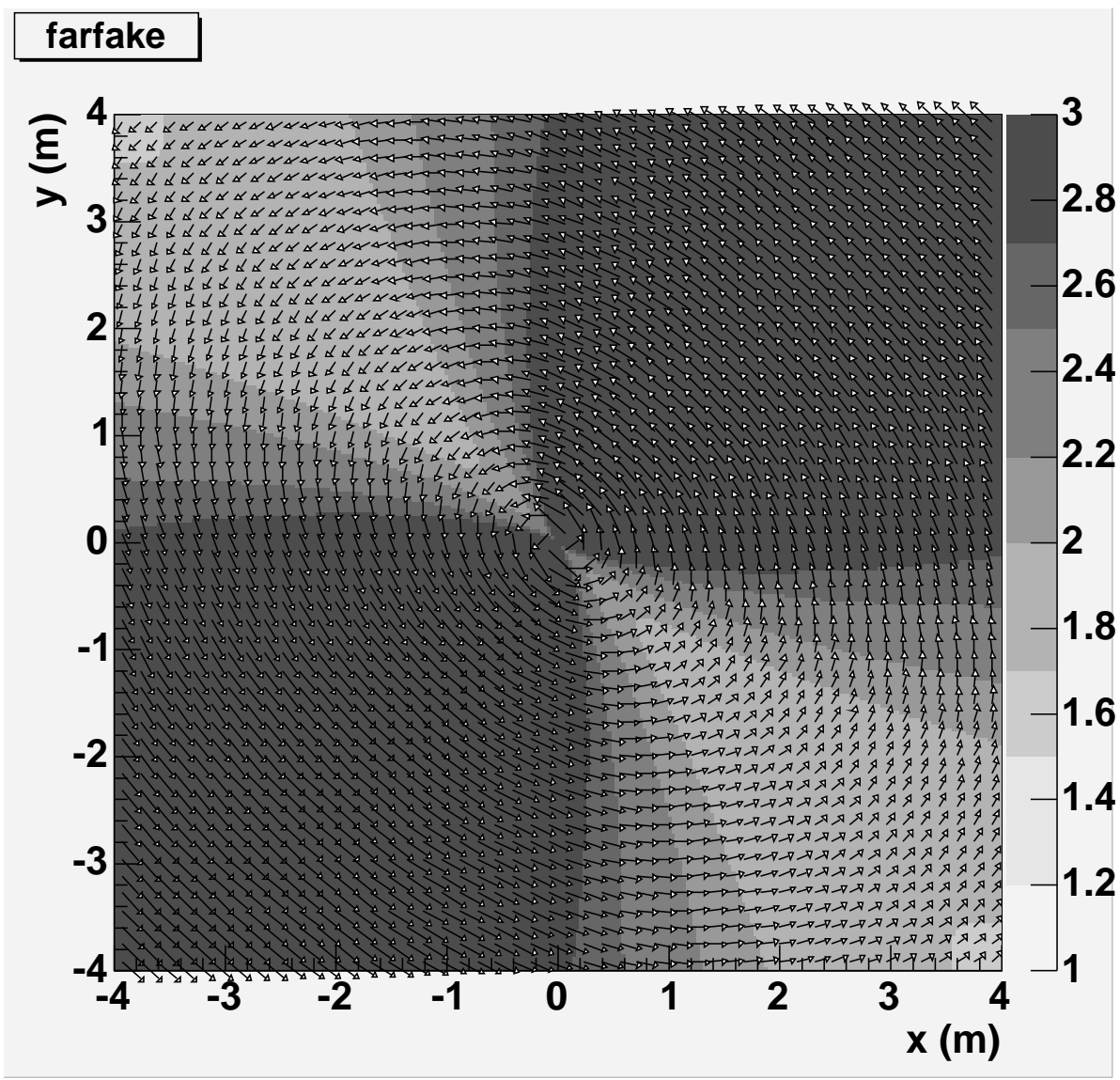

Figure 6.14: A Map of the Modified Magnetic Field used with the Toy Monte Carlo in Tesla. 


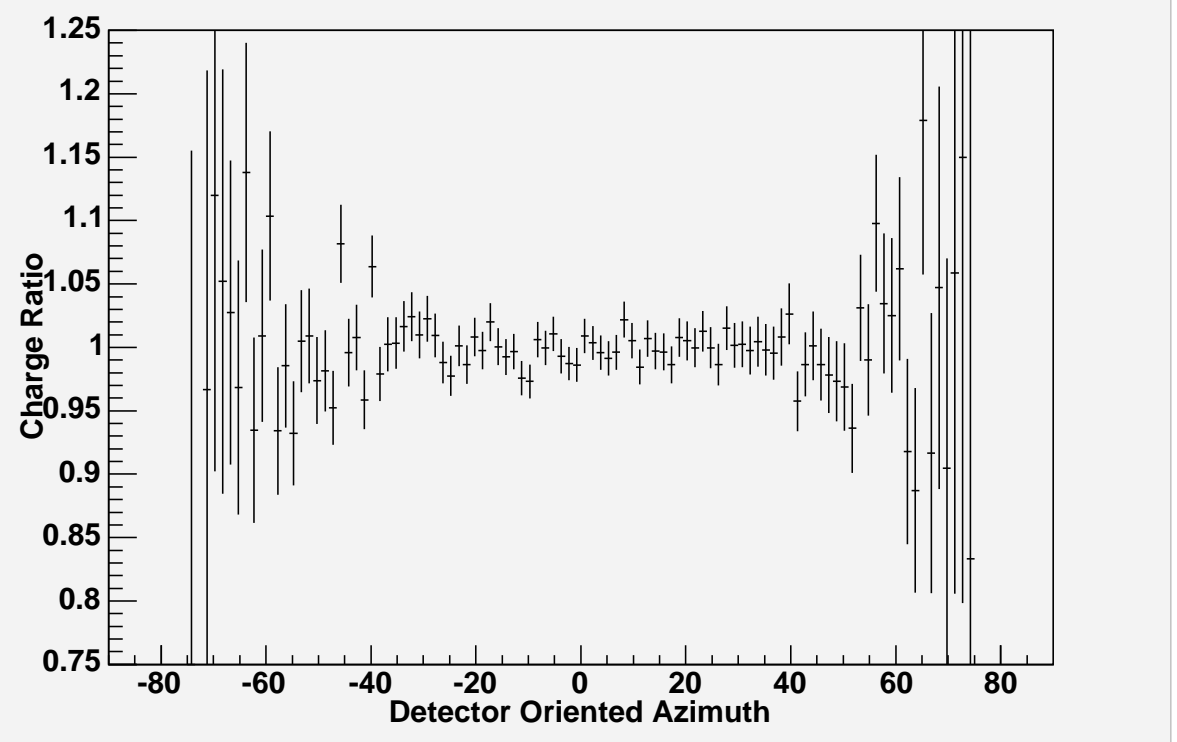

Figure 6.15: The charge ratio as a function of detector azimuth for the Toy Monte Carlo, using the modified magnetic field. The sine wave variation in the charge ratio of Figure 5.13 is not present. 
Now, individual steel planes may deviate from the actual coordinate system in $X, Y$ by as much as a centimeter or so, due to construction tolerances such as the steel lamination cutting and welding. Each steel plane that is instrumented will have scintillator plane that is offset in $X, Y$ by very small amount, since these are lined up more carefully after the steel sheet has been placed. Given the close tolerances required, deviations must be of order millimeters and not centimeters or the coil collar would not fit.

Next, once in position as part of the detector, the $\mathrm{Z}$ position of the South face of each steel sheet is set very precisely (tenth of millimeter machining) by the collar at the center. Unfortunately, the eight corners of the steel plane may have deviations in $\mathrm{Z}$ by as much as a few millimeters. The reason is that the collar rests against the previous collar, and so is not sensitive to non-standard thicknesses of steel planes, while the axial bolts at each corner are rested against the previous steel plane, so they are sensitive to non-nominal thicknesses of steel. The upshot of this is that while the centers of the steel planes move in a well defined manner, the eight corners do not, and so the steel could be chopped into "octants" which all move in $\mathrm{Z}$ in different ways. One caveat about the collars and axial bolts: there are a small number of "yellow" collars and axial bolts that are machined down by 3.2 millimeters that were placed in unrecorded places inside the detector. They were sprayed with yellow paint to distinguish them, so the shortened axial bolts can be determined from visual inspection, but the collars are not visible from the outside, and the detector cannot be disassembled easily.

Finally, the scintillator modules rested against the steel do not rest equally flat against the steel at all points, but rather are closest touching in the centers of the module and raised at the edges where the steel clips are located by one to three millimeters. 
Understanding of these "misalignments" of the scintillator are difficult, and in particular the yellow collar problem will require further work to untangle. 


\section{Chapter 7}

\section{Measurement of the Charge Ratio}

\subsection{Charge Biases in the Data}

Due to the charge-dependent acceptance of the detector, the charge ratio is about $10 \%$ higher from the North than from the South with the field in the Forward configuration. Since the muon flux is about $10 \%$ larger from the South, the charge ratio will tend to be lowered if all charge ratio data points were taken together. This is termed an illumination bias, which is one bias that would be removed totally by summing Forward and Reverse field data sets.

It is known that there are biases in the detector's curvature response. By reversing the field, any bias affects the opposite charge in the same way, providing a way to cancel such biases.

(1) Assume that for a bin of some azimuth and zenith angle, there are either some number of positive muons incorrectly reconstructed as negative, or some number of negative muons incorrectly reconstructed as positive. In practice, there will be both situations going on at the same time, but taking all biases together one will be left with a single number. This number is the bias, termed A, which either operates in one way to increase the appearance of tracks of detectable positive curvature and decrease the number of tracks 
of detectable negative curvature or else it operates in the opposite direction. For the sake of the following discussion, assume that this bias acts to increase the positive curvature signal and cause a loss in the negative curvature signal. A is the bias, or some percentage of the total signal, which can be seen from the original problematic charge ratio plots in Chapter 5 such as the detector azimuth plot to be as much as $5 \%$.

$$
\begin{aligned}
& \mu_{\text {Forward }}^{+}=\mu_{\text {True }}^{+} \times(1+A) \\
& \mu_{\text {Forward }}^{-}=\mu_{\text {True }}^{-} \times(1-A)
\end{aligned}
$$

(2) Assume that the field inverts everywhere inside the detector when the current in the coil is reversed. The bias A is now the bias (for that same azimuth and zenith angle bin) that increases the negative curvature signal at the sake of the positive curvature signal and is exactly the same size as the bias A in Equation 7.1.

$$
\begin{aligned}
& \mu_{\text {Reverse }}^{+}=\mu_{\text {True }}^{+} \times(1-A) \\
& \mu_{\text {Reverse }}^{-}=\mu_{\text {True }}^{-} \times(1+A)
\end{aligned}
$$

(3) If you normalize the size of the Forward and Reverse data samples and add them together, the ratio will give the true charge ratio.

$$
\begin{aligned}
R & =\frac{\mu_{\text {Forward }}^{+}+\mu_{\text {Reverse }}^{+}}{\mu_{\text {Forward }}^{-}+\mu_{\text {Reverse }}^{-}} \\
& =\frac{\mu_{\text {True }}^{+}+\mu_{\text {true }}^{+} \times A+\mu_{\text {True }}^{+}-\mu_{\text {True }}^{+} \times A}{\mu_{\text {True }}^{-}-\mu_{\text {true }}^{-} \times A+\mu_{\text {True }}^{-}+\mu_{\text {true }}^{-} \times A} \\
& =\frac{\mu_{\text {True }}^{+}}{\mu_{\text {True }}^{-}}
\end{aligned}
$$

There are three other known possible sources of systematic error after the summing procedure is used: 
- The bias elimination procedure rests on the assumption that the field reverses everywhere exactly when the current is flipped. The steel is completely saturated at up to nearly 3.5 meters from the coil hole, so it seems reasonable that a large applied field would mask any knowledge (hysteresis) of past fields. This assumption can be tested by examining the $\chi^{2}$ of a flat fit to a distribution that is expected to be flat.

\begin{tabular}{|l|c|c|c|c|}
\hline Sample & Charge Ratio & $\chi^{2}$ & MC Charge Ratio & MC $\chi^{2}$ \\
\hline \hline Forward & 1.384 & 44.6 & 1.244 & 1.21 \\
Reverse & 1.292 & 30.2 & 1.253 & 1.05 \\
Summed & 1.347 & 1.03 & 1.249 & 1.08 \\
\hline
\end{tabular}

Table 7.1: The $\chi^{2}$ of a fit to the Charge Ratio versus Detector Azimuth for Forward and Reverse field data, and also after the summing procedure.

The $\chi^{2}$ obtained after the summing procedure in Table 7.1 indicates the original systematic effect cancels so the field did invert uniformly.

- As was seen in Chapter 4, a track observed in the detector going through a region with misalignment on the order of cm could be of high momentum, but be reconstructed as having a large curvature. If the misalignment preferentially gives one charge sign over the other for these high energy muons with little real curvature, then this is a bias. On the other hand, if the misalignments are more random and give the track the appearance of having more multiple scattering, then the resulting charge identification will include more randomly determined charge and this misalignment gives rise to a background, or impurity of the charge separated sample with randomly assigned charges. This background will tend to pull the charge ratio towards 1.0. This will be discussed below. 
- The reconstruction software must begin with a guess of the curvature and a negative charge sign. The negative charge was chosen since MINOS would be focussing negative muons from the direction of Fermilab. It is possible that the Kalman filter would tend to converge more often on a negative curvature than a positive curvature due to the initial state biasing towards the negative. This was examined by reconstructing data muon tracks with a field map in the forward and also in the reverse direction to get two reconstructed data sets. The forward and reverse field positives were summed, as were the forward and reverse field negatives. If there were a bias, it would show up very clearly as a deviation from 1.0 in the charge ratio plot of these data sets, which is shown in Figure 7.1. The $\chi^{2}$ of a flat line fit is 0.73 , with a fit value of 1.005 . So there is some reproducibility issues with reversing the field in the reconstruction software, with a magnitude of $0.5 \%$.

It is important to note that if both configurations of reconstruction on the same DAQ data are done on the same computing node, this nonreproducibility goes away and the resultant data sets are exactly complementary. The underlying behavior is not understood but is known to be dependent on what computing node does the processing. The Fermilab computing farm for MINOS contains over 100 nodes and processing in parallel is the only way to reconstruct the data in a timely manner (roughly one year to reprocess all the Far Detector data on one dual $2 \mathrm{GHz}$ node).

\subsection{Summing the Complementary Data Sets}

The difference in time between the end of a particular run in one of the two "Field Configurations" and the beginning of that same run was defined as 


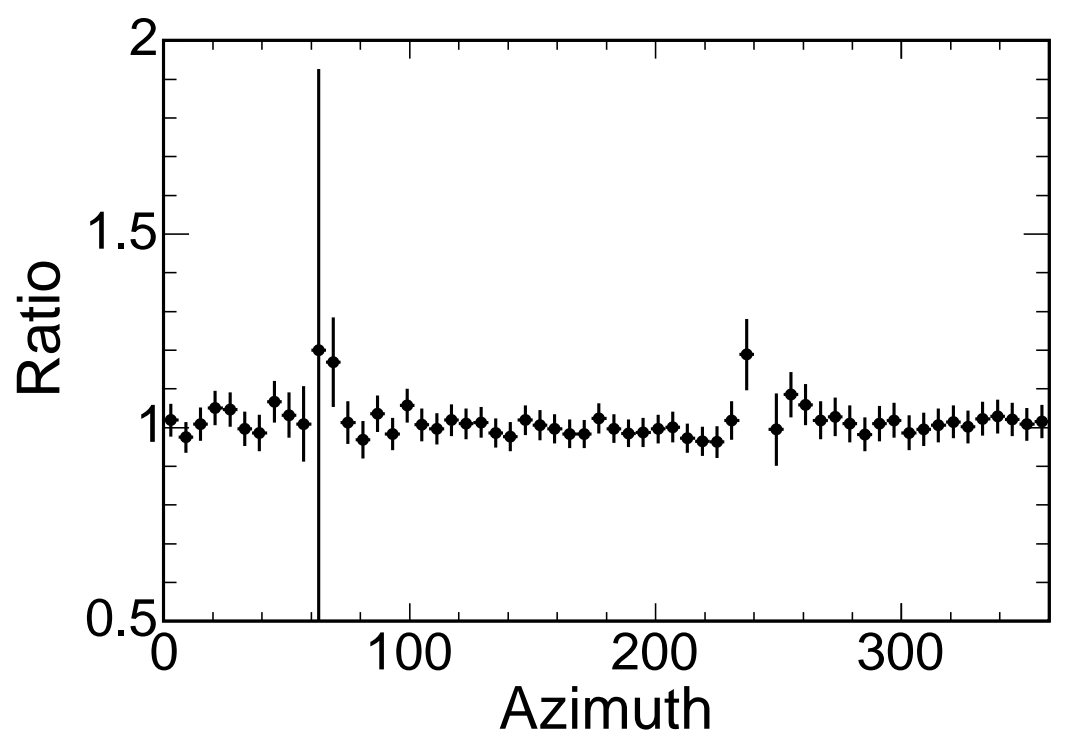

Figure 7.1: The Charge Ratio as a function of azimuth for summing the same data with both field configurations. 
$T_{\text {Run Field Config }}$. Livetime for a particular Field Configuration was then the sum of all runs that passed the run rate cut: $\Delta T_{\text {Forward }}=\Sigma T_{\text {Run }}$ Forward $_{\text {. After the histograms }}$ of events passing cuts were built up, the Forward data set histograms were normalized to the livetime of the Reverse data set by multiplying by $\Delta T_{\text {Reverse }} / \Delta T_{\text {Forward }}=$ $29,643,581 s / 17,249,414 s=0.5819$. After the normalization, a histogram for positive muons in the Forward set could be summed with a like histogram for positive muons in the Reverse set to remove the charge identification biases. The same is done for the negative muon histograms and then a charge ratio histogram is simply the ratio of the summed positive muon histogram over the summed negative muon histogram. This was also done for the two Monte Carlo sets which were generated and reconstructed in each of the two magnetic field configurations.

The azimuthal, zenith and azimuthal angle about the z-axis charge ratio plots are shown in Figures 7.2-7.5.

The summing procedure is observed to remove the East/West charge bias in the data, and the North/South bias due to focussing. It is very important to note that the charge ratio as a function of either dcosz or zenith is not expected to be flat, since the average slant depth changes as dcosz or zenith changes. Since the Monte Carlo has a flat charge ratio as a function of overburden, and the data may have a changing charge ratio, an expectation of the charge ratio versus dcosz and zenith can be explored by reweighting the Monte Carlo events.

\subsubsection{Monte Carlo and Reweighting}

Because the charge ratio data may have a dependence on slant depth, the Monte Carlo as generated can not be used for direct comparison unless it were to have this same dependence. The Monte Carlo events were reweighted one by one to give a charge ratio versus slant depth matching the data. Now an expectation of 


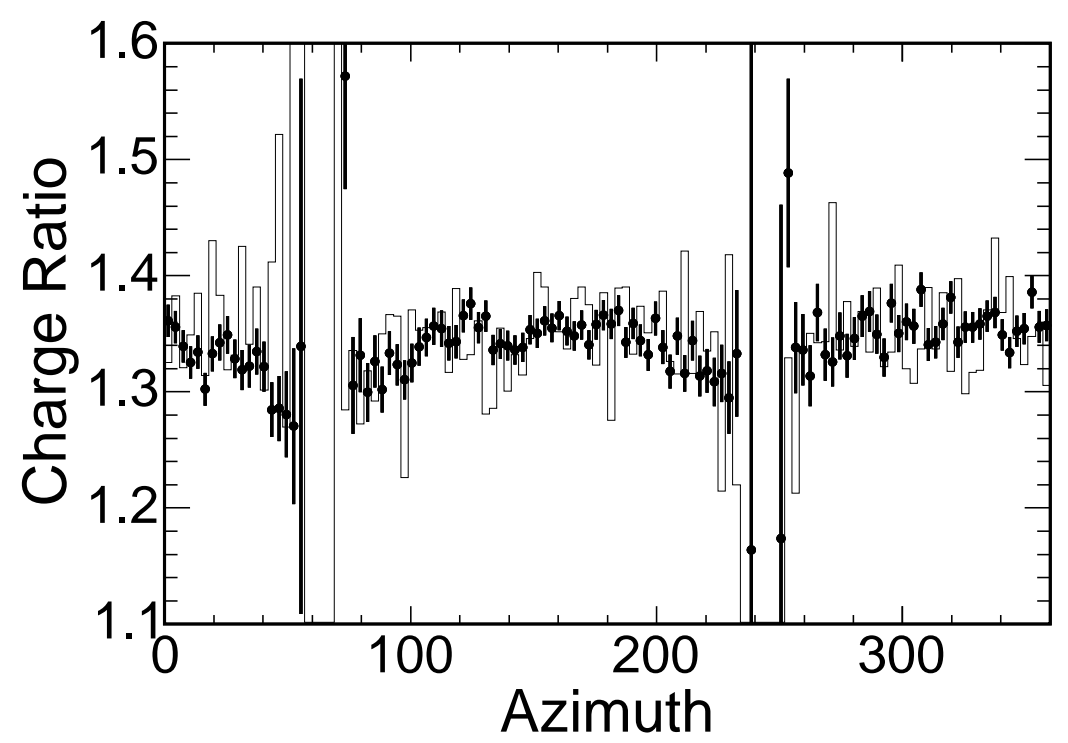

Figure 7.2: The Charge Ratio as a function of azimuth for summed data sets, Data is with error bars, Monte Carlo is continuous line wherein the $\mathrm{MC}$ charge ratio has been scaled to a charge ratio of 1.35 for visual comparison with data.

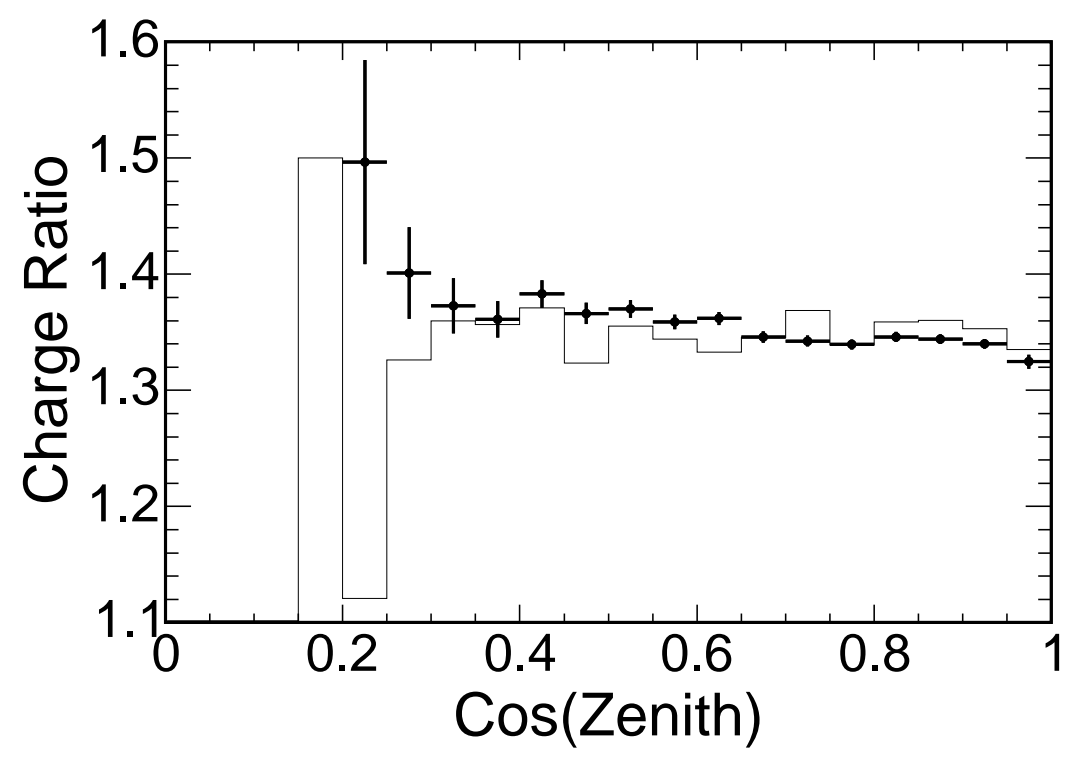

Figure 7.3: The Charge Ratio as a function of zenith for summed data sets, Data is with error bars, Monte Carlo is continuous line wherein the $\mathrm{MC}$ charge ratio has been scaled to a charge ratio of 1.35 for visual comparison with data. 


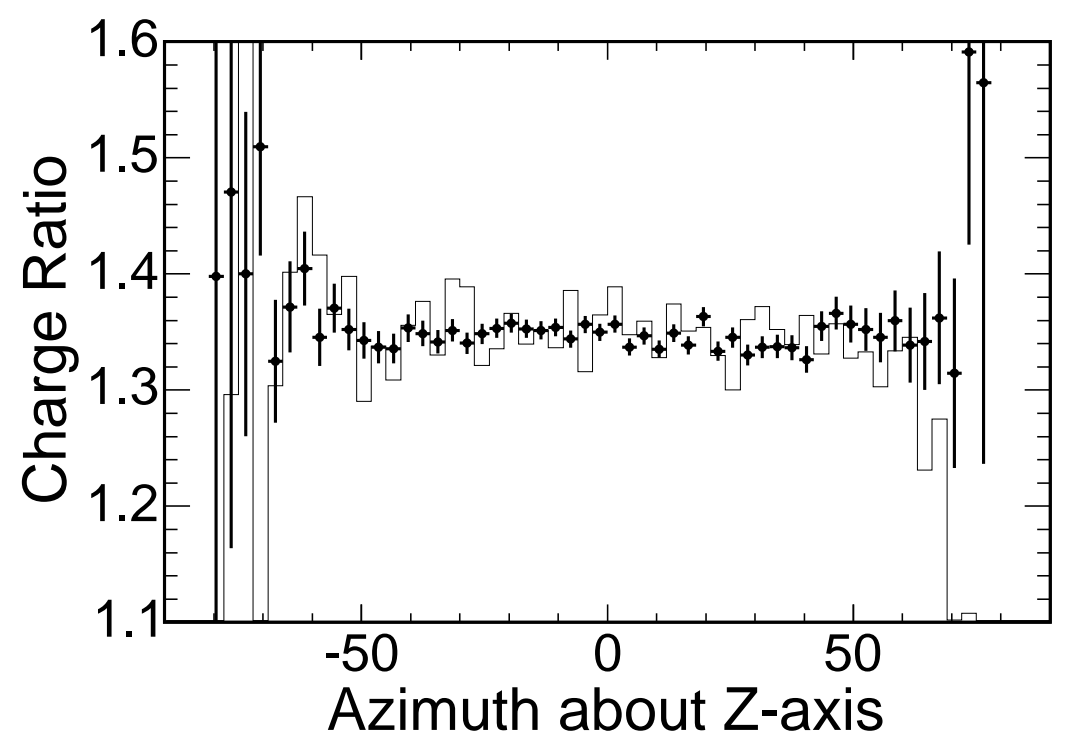

Figure 7.4: The Charge Ratio as a function of the angle in the XY plane, about the Z-axis, for summed data sets, Data is with error bars, Monte Carlo is continuous line wherein the MC charge ratio has been scaled to a charge ratio of 1.35 for visual comparison with data.

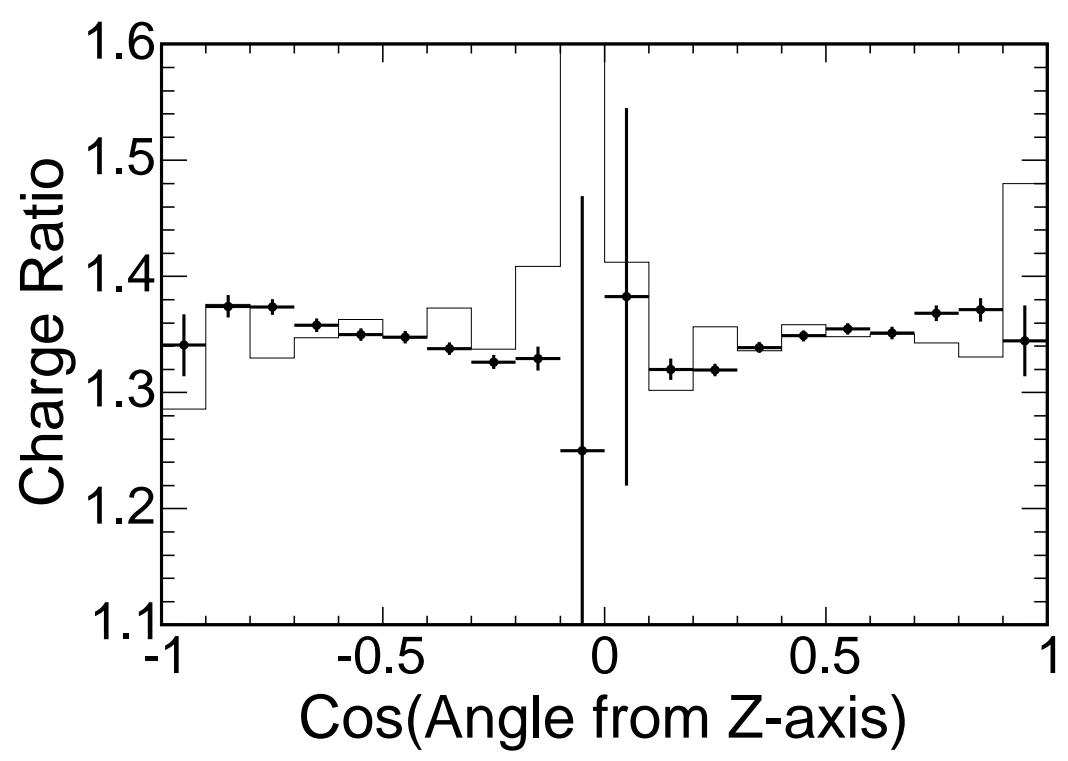

Figure 7.5: The Charge Ratio as a function of the angle from the Z-axis that the muon is travelling towards, for summed data sets, Data is with error bars, Monte Carlo is continuous line wherein the MC charge ratio has been scaled to a charge ratio of 1.35 for visual comparison with data. Not expected to be flat. 
various slant depth dependent plots can be made.

There is now a dependence on dcosz after reweighting by slant depth. The Monte Carlo was also reweighted to follow the same dependence as in Figure 7.5. The slant depth dependence of charge ratio was compared with the data, and a correlation is seen. All that can be certain at this point is that charge ratio has a dependence on dcosz and slant depth, but whether the underlying behavior is detector efficiency or physics can not be determined at this time. Future studies must find a way to disentangle the two dependencies.

\subsection{Slant Depth}

Using the overburden determined from the elevation model and the Soudan 2 density data, the charge ratio can be compiled into bins in slant depth in m.w.e. An integrated charge ratio and the average slant depth it measures can also be obtained.

The charge ratio as a function of slant depth is given as $\mathrm{R}(\mathrm{X})=1.300 \pm 0.008$ $+(1.8 \pm 0.3) \times 10^{-5} \times X$ (stat). The charge ratio integrated over all slant depths is $R_{e f f}=1.346 \pm 0.002$ (stat). The average slant depth for this result is 2500 M.W.E.

\subsection{Surface Energy}

Using the method outlined in Chapter 2, the slant depth can be converted into muon surface energy. The accuracy on the method was quoted as 5\%, but this was based solely on the range of energy measured at MINOS. Due to the large deviations involved in muon propagation through matter, the result cannot be used in compilations of muon charge ratio when surface energy is used until simulations determine the accuracy of the slant depth conversion into surface energy.

The charge ratio as a fuction of surface energy is $R\left(E_{\mu}\right)=1.327 \pm 0.004+$ 


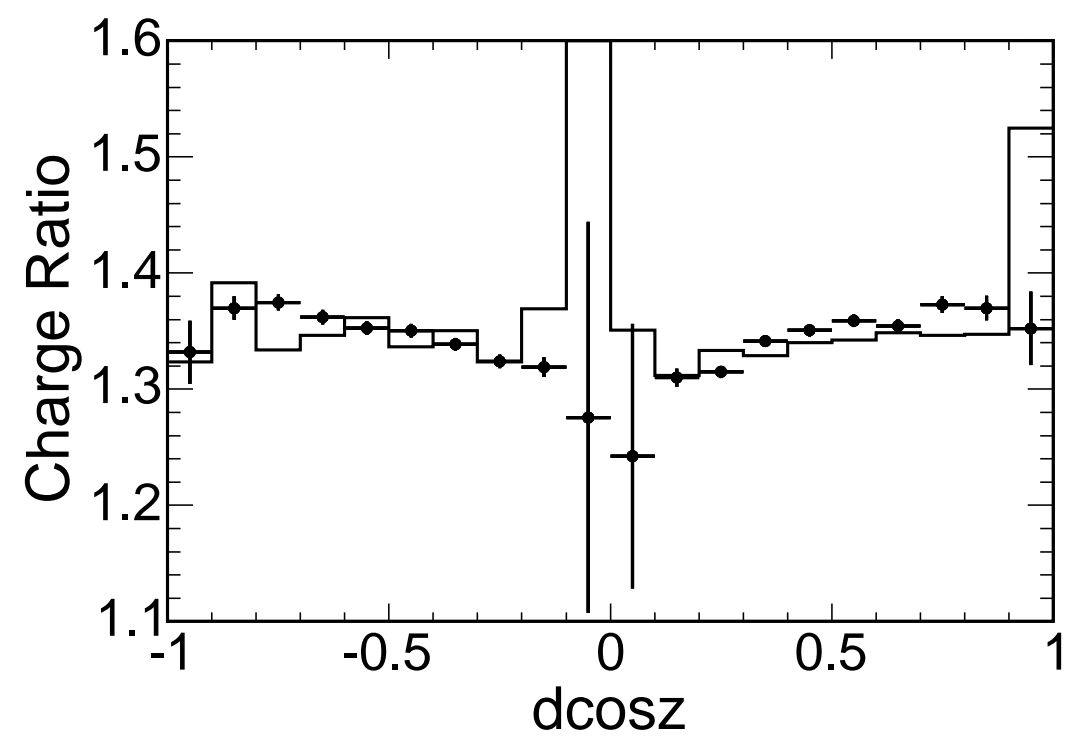

Figure 7.6: Plot of Figure 7.5, after reweighting to contain the same charge ratio dependence on slant depth. MC is solid line, data is filled circles.

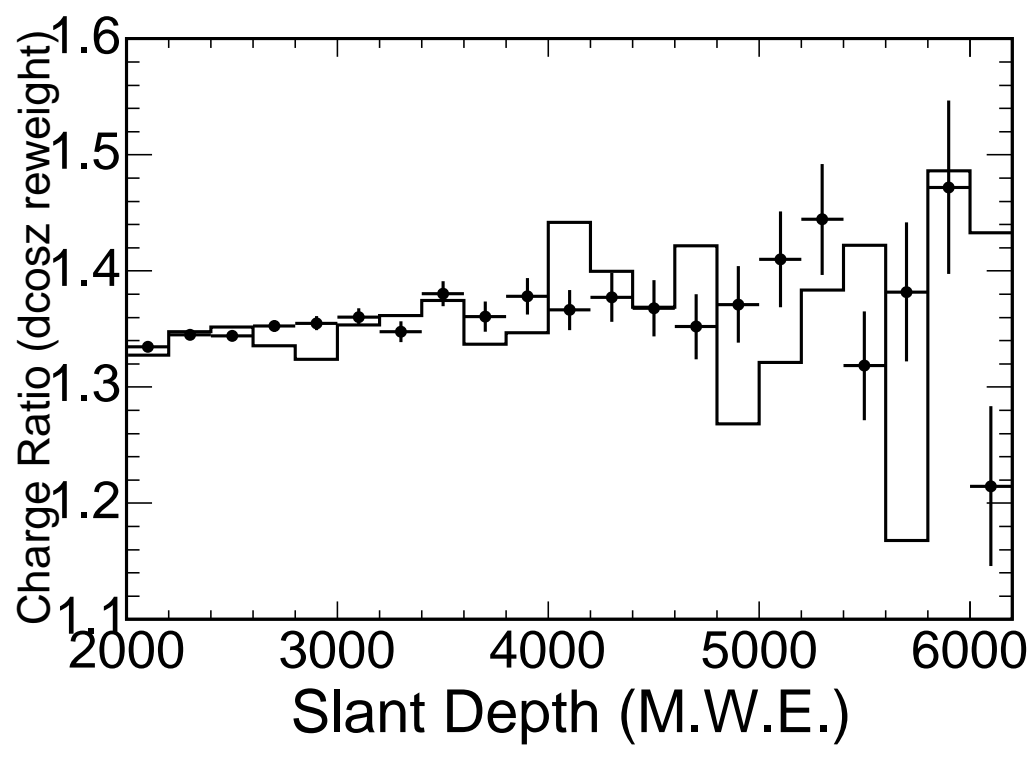

Figure 7.7: Plot of charge ratio versus slant depth, after reweighting by dcosz to contain the same charge ratio dependence on dcosz as in data. MC is solid line, data is filled circles. 


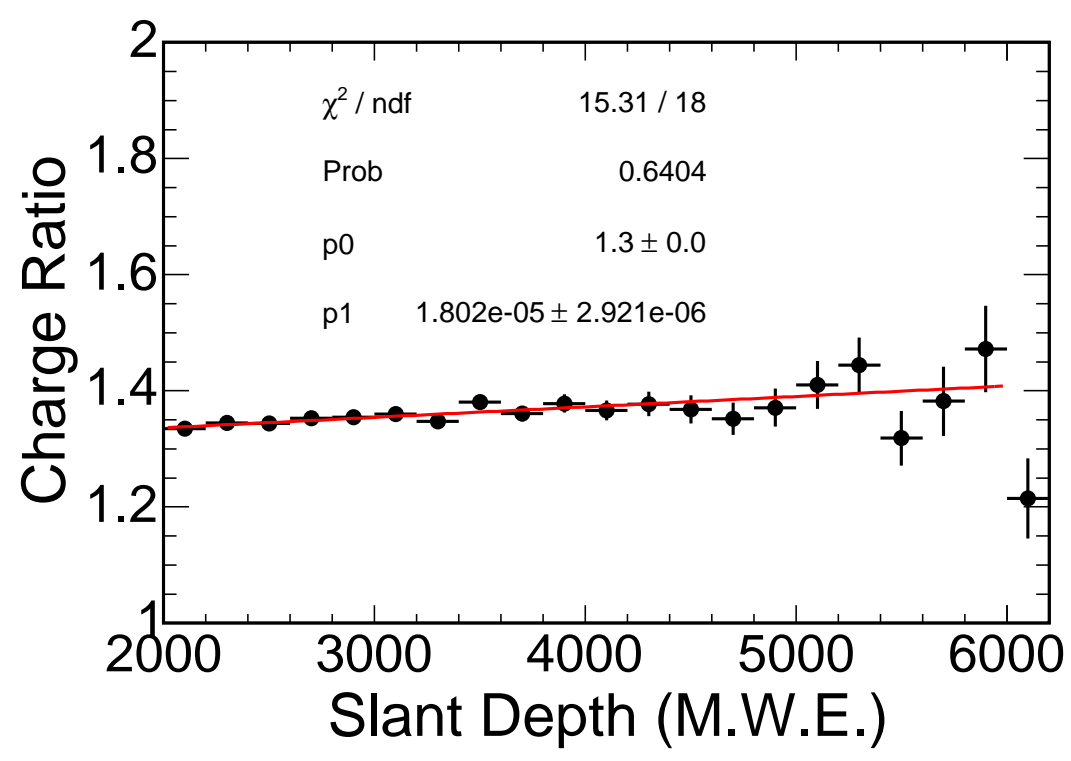

Figure 7.8: The Charge Ratio as a function of slant depth after the summing procedure.

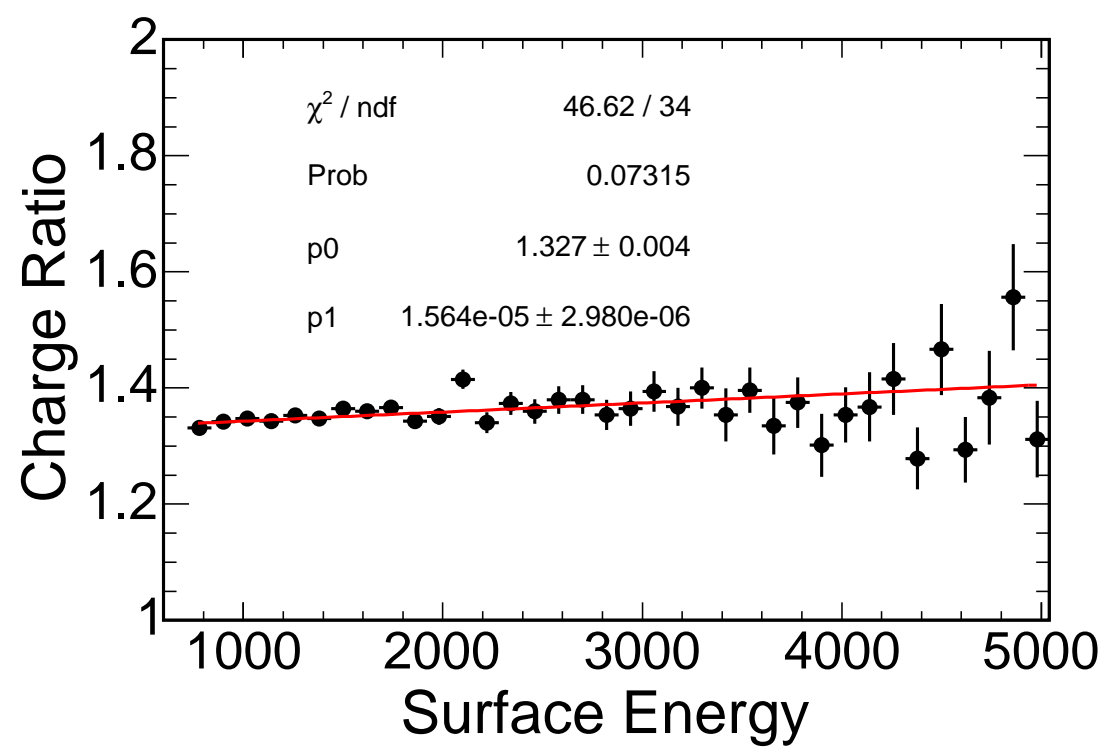

Figure 7.9: The Charge Ratio as a function of surface muon energy after the summing procedure. 
$(1.5 \pm 0.3) \times 10^{-5} \times E_{\mu}$. The integrated charge ratio is the same as that for slant depth, $R_{e f f}=1.346 \pm 0.002$ (stat), with an average surface energy for this result of $890 \mathrm{GeV}$. 


\section{Chapter 8}

\section{Systematic Error}

By reversing the magnetic field, the MINOS data set is free of systematic errors that tend to push the charge ratio towards a biased value and away from the "true" value. By making a cut of charge identification confidence and summing the forward and reverse data sets normalized by livetime, illumination biases and any charge identification biases cancel out. No other recent experiment does this in a search for charge ratio of cosmic muons.

However, there are systematics that may push the charge ratio towards a value of 1.0 by including muon tracks that have essentially random charges, primarily very straight tracks that have just enough multiple scattering to pass the charge identification cut. This randomization will pull the value towards 1.0 and so a study must be done of its effect. The randomization problem inherent in any kind of measurement with background like this leads to lower charge ratios. There are many ways to get the charge ratio too low, but few ways to get it higher.

The systematic error has been examined from three perspectives:

- a time dependence of the MINOS detector's charge determination,

- any dependence on cut sample or biases in the analysis,

- and the flatness of distributions after the summing procedure, given in Chap- 
ter 7, Table 7.1 .

\subsection{Time Dependence of Charge Ratio Measurement}

Reverse charge ratio was higher than input because of the flux effect of North versus South-going muons. A further correction was needed because the detector's response changed over time. A gradual and nearly constant drop in event rate required two sets of summing the data to fully account for the possibility this would provide a further bias. The magnetic field was in the forward configuration for nearly one year before being flipped, and nine months later, it was flipped back to forward. The data can thus be divided up into four sets, or two sets of summed data: forward initial and reverse first half, reverse second half and forward second half. The proportions of livetime in each of the four samples was far from uniform, which presents a further test of the summing procedure's statistical dependence. The samples were as follows:

(1) Forward field July 2003 - June 2004,

(2) Reverse field June 2004 - October 2004,

(3) October 2004 - February 2005,

(4) February 2005 - April 2005.

Sample 1 was summed with Sample 2, weighted by 2.65 to make the first data set. Sample 3, weighted by 0.781 , was summed with Sample 4 to make the second data set. The charge ratio as a function of slant depth as found and fit for each sample and the results are plotted in Figures 8.1 and 8.2.

The difference between fits leads to a contribution to the overall systematic error of $0.4 \%$ to the integrated charge ratio. 


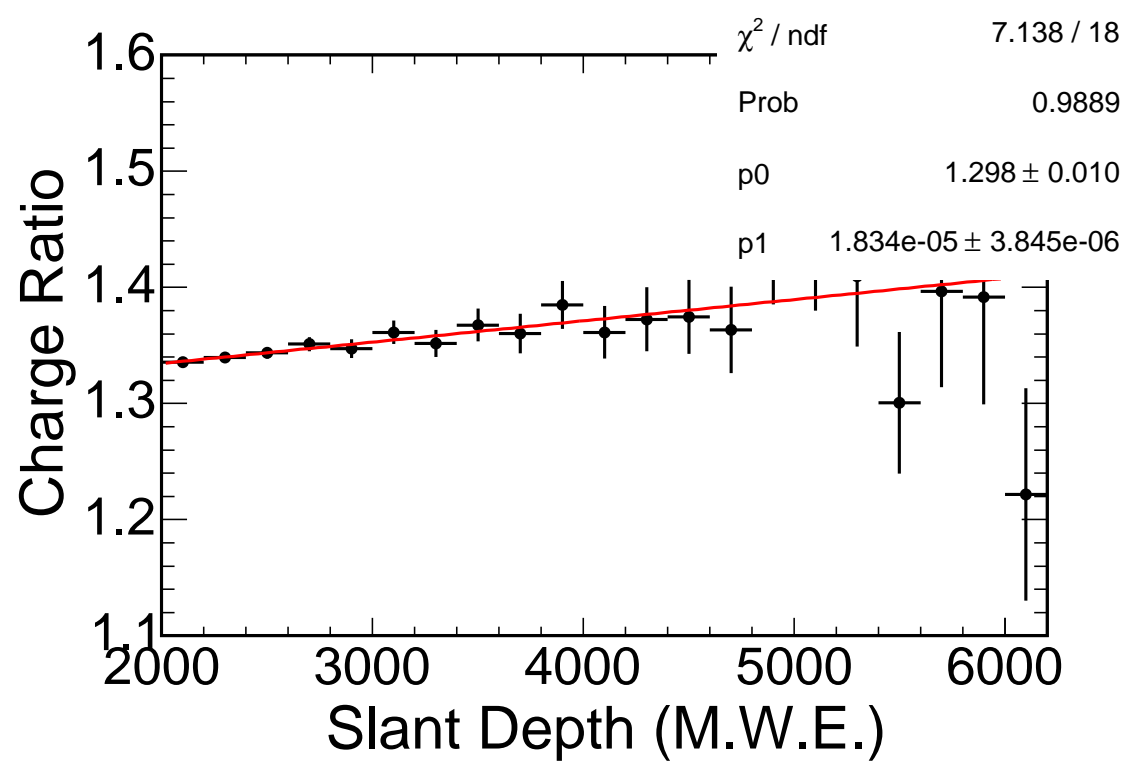

Figure 8.1: The Charge Ratio fit versus slant depth for Sample 1.

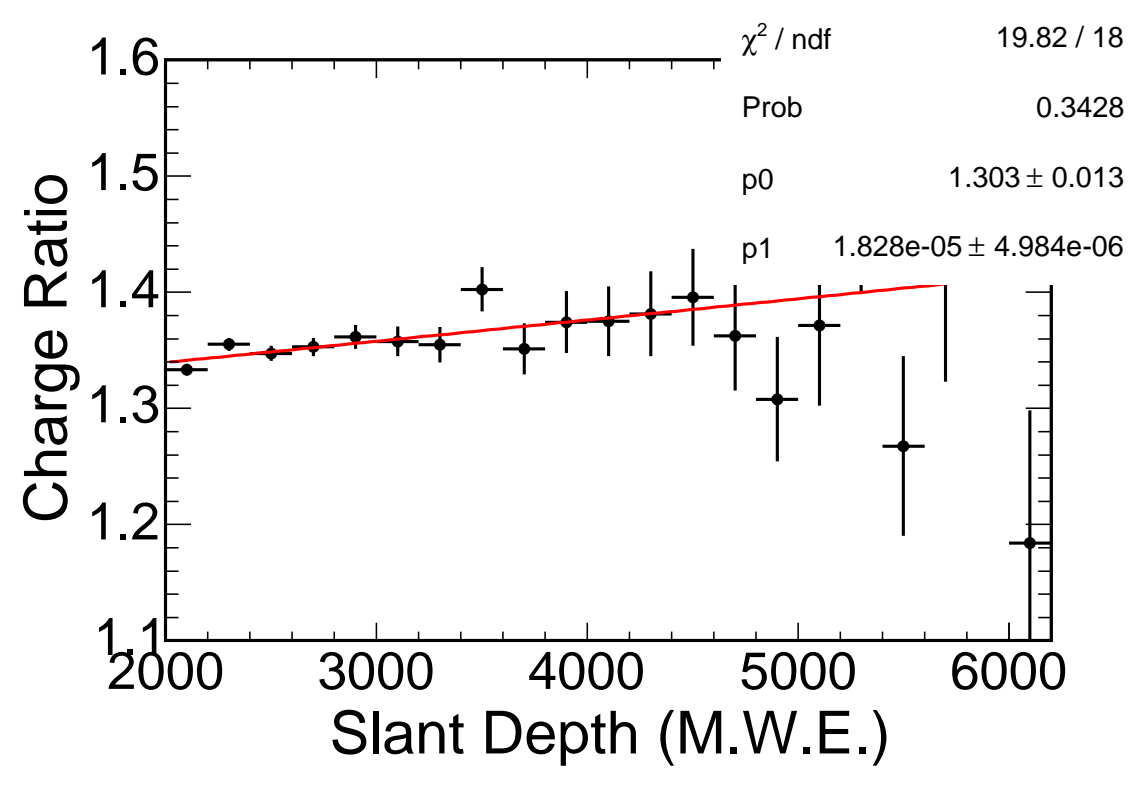

Figure 8.2: The Charge Ratio fit versus slant depth for Sample 2. 


\subsection{Analysis Cuts Dependence of Charge Ratio Measurement}

The reweighted Monte Carlo can be used to generate an expectation of the charge ratio and thereby determine the systematic error on the measurement. Reweighted MC has the slant dependence of the data, and any differences between this MC and the data is the systematic error contribution from the analysis. The charge ratio was seen to have dependencies on plane number, track length and track vertex dcosz, so the total charge ratio was calculated for various cuts in data and the reweighted MC. The percent difference in Charge ratio, data minus the MC was then plotted as a function of the cut in Figure 8.3.

- The selection of plane cut changes the charge ratio by $0.9 \%$.

- Length cut affects charge ratio by $0.2 \%$.

- The dcosz cut affects charge ratio by $0.5 \%$.

\subsection{Conclusion}

The contributions of the time dependence investigation, the cuts analysis, and the the $0.5 \%$ reproducibility error from Section 7.1 were added in quadrature to get a total systematic error of $1.2 \%$.

Thus the measured charge ratio is $R_{e f f}=1.346 \pm 0.002$ (stat) \pm 0.016 (syst) and the slant depth fit is $R(X)=1.300 \pm 0.008$ (stat) \pm 0.016 (syst) $+(1.8 \pm 0.3) \times$ $10^{-5} \times X$, which is valid over the range $2000<\mathrm{X}<6000$ MWE. 

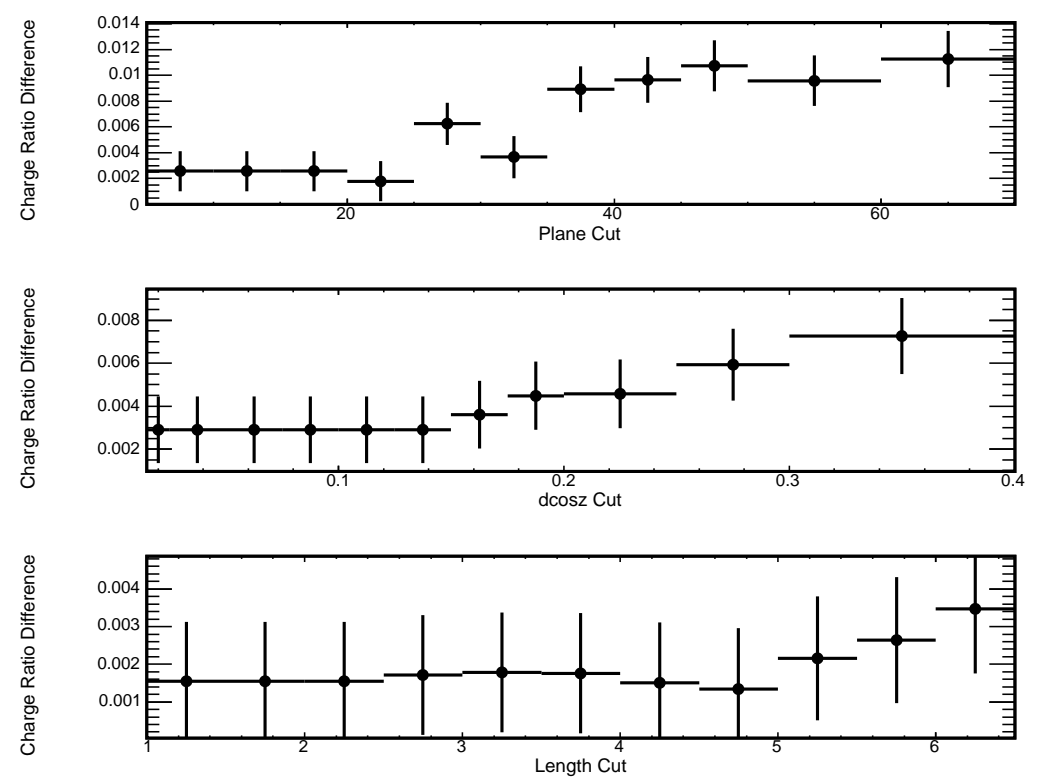

Figure 8.3: The integrated Charge Ratio as a function of cuts on plane number, track length and track vertex dcosz. 


\section{Bibliography}

[1] S. Eidelman et al. Review of Particle Physics. Physics Letters B, 592:1+, 2004.

[2] J.A. Simpson. Ann. Rev. Nucl. and Part. Sci., 33:323, 1983.

[3] Sue Kasahara. A Study of Cosmic Ray Composition in the Knee Region using Multiple Muon Events in the Soudan 2 Detector. PhD thesis, University of Minnesota, 1997.

[4] David M. DeMuth. A Search for Neutrinos from Active Galactic Nuclei. PhD thesis, University of Minnesota, 1999.

[5] B. Vulpescu et al. J. Phys. G: Nucl. Part. Phys., 27:977, 2001.

[6] The L3 Collaboration. Measurement of the atmospheric muon spectrum from 20 to 3000 gev. Phys. Lett. B, page 32, 2004, hep-ex/0408114.

[7] Brian J. Rebel. Neutrino-Induced Muons in the MINOS Far Detector. PhD thesis, Indiana University Bloomington, 2004.

[8] Atmospheric Muons and Neutrinos. Proceedings of 2nd Workshop on Methodical Aspects of Underwater/Underice Neutrino Telescopes, Hamburg, 2001, hep-ph/0201310.

[9] Gaisser. Cosmic Rays and Particle Physics. Cambridge University Press, Melbourne, 1990.

[10] V.A. Naumov. private communication. July 2005.

[11] A. Para. Private communication to stuart mufson and brian rebel. July 2005.

[12] K. Ruddick. Some notes on underground muons. PDK 435, May 1990.

[13] J.D. Jackson and R.L. McCarthy. $z^{3}$ corrections to energy loss and range. Phys.

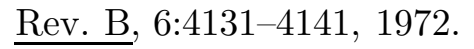

[14] K.E. Eriksson et al. Il Nuovo Cimento, 30:1434, 1963.

[15] Hans-Jochen Trost. On the scattering of muons in the rock above soudan 2. PDK 500, January 1992. 
[16] Hans-Jochen Trost. Simulation of the passage of muons through the rock overburden into the soudan 2 cavern. PDK 473, July 1991.

[17] Proceedings of the 20th International Cosmic Ray Conference, 1987.

[18] T. Hebbeker and C. Timmermans. A compilation of high energy atmospheric muon data at sea level. Astropart. Phys., 18:107-127, 2002, hep-ph/0102042.

[19] J. Kremer et al. Measurements of ground-level muons at two geomagnetic locations. Phys. Rev Lett, 83:4241-4244, 1999.

[20] HE 3.2-3.4: Neutrinos and Muons. Interactions, Particle Physics Aspects, Astro-Particle Physics and Cosmology, 2003, hep-ph/0311289.

[21] B. Rebel and S. Mufson. A demultiplexing algorithm for cosmic rays in the minos far detector. NuMI 834, April 2002.

[22] R. Lee N. Felt, A. Lebedev and J. Oliver. Minos far detector electronics -user's manual-. NuMI 901, January 2003.

[23] Caius L. F. Howcroft. Atmospheric Neutrinos in the MINOS Far Detector. PhD thesis, Cambridge University, 2004.

[24] R. Lee. A description of the minos reconstruction framework. NuMI 916, April 2003.

[25] Ryan Nichol. Calibration of the MINOS Detectors. PhD thesis, University College London, 2003.

[26] David A. Petyt. A Study of Parameter Measurement in a Long Baseline Neutrino Oscillation Experiment. PhD thesis, University of Oxford, 1998.

[27] A. Para P. Adamson and J. Thomas. Performance of a least-squares based muon reconstruction procedure for minos. NuMI 536, August 1999.

[28] A. Para J. Thomas and D. Tovee. The principles of muon tracking in minos. NuMI 301, October 1997.

[29] Geodetic Determinations for the NuMI Project at Fermilab. Proceedings of the 6th International Workshop on Accelerator Alignment IWAA 99, Grenoble, 1999.

[30] B. Becker and D. Boehnlein. Alignment of the minos far detector. NuMI 1047, November 2004.

[31] M. Goodman P. Schreiner, V. Frohne and T. Fields. Minos far detector acceptance study for understanding aspects of the mufson effect. NuMI 1049, September 2004.

[32] D. et al Heck. Evidence for neutrino oscillations from muon decay at rest. Phys.

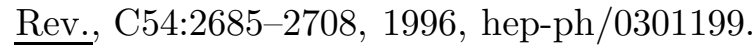


[33] E.V. Bugaev et al. Atmospheric muon flux at sea level, underground and underwater. Phys. Rev. D, 58:05401, 1998, hep-ph/9803488.

[34] A.R. Clark et al. Phys. Letters B, 41:229, 1972.

[35] J. Kilmer D. Boehnlein, R. Hatcher and J. Nelson. Steel mass and meltcode distribution in the minos far detector. NuMI 1061, November 2004.

[36] R. Pitiera B. Becker and A. Sousa. Surveying the construction of super module 2. NuMI 964, September 2003.

[37] W. Miller T. Ladran, J. Alner and E. Peterson. Assessment of the minos steel design. NuMI 949, August 2003.

[38] E. Peterson J. Kilmer J. Meier, J. Nelson. Minos plane installation and removal procedure - soudan. NuMI 919, April 2003.

[39] MINOS collaboration. The MINOS detectors Technical Design Report. NuMI 337, October 1998.

[40] R. Hatcher. Numbering conventions and coordinate systems in gminos. NuMI 243, February 1997.

[41] G. Feldman A. Lebedev J. Oliver, N. Felt and R. Lee. Design and performance of the readout system of the minos far detector. IEEE Trans. Nucl. Sci., 51:21932195, October 2004.

[42] E. Beall. Documentation of minos detector control systems: Design and implementation. NuMI 1089, September 2004. 